\title{
C- to N-center Remote Heteroaryl Migration via Electrochemical Initiation of N Radical by Organic Catalyst
}

Chengkou Liu, ${ }^{\dagger}$ Qiang Jiang, ${ }^{\dagger}$ Yang Lin, ${ }^{\dagger}$ Zheng Fang, ${ }^{\dagger}$ and Kai

$$
\mathrm{Guo}^{*,+, \neq}
$$

${ }^{\dagger}$ College of Biotechnology and Pharmaceutical Engineering, Nanjing Tech University, Nanjing, 211816 China

Ftate Key Laboratory of Materials-Oriented Chemical Engineering, Nanjing Tech University, Nanjing, 211816 China

E-mail: guok@njtech.edu.cn. 
Table of Contents

1. General Remarks $\quad$ S3

2. Synthesis of the Starting Materials and Catalysts S4-S7

3. General Procedure for the Electrochemical $\begin{array}{ll}\text { Heteroaryl Migration } & \text { S7 }\end{array}$

4. Gram-Scale Synthesis of $\mathbf{2 b} \quad$ S8

$\begin{array}{ll}\text { 5. Mechanistic Studies } & \text { S8-S10 }\end{array}$

6. Structure of $\mathbf{2 f}$ by X-Ray Crystallographic (CCDC: 1955548)

7. Characterization Data of and Starting Materials 1, Catalysts and Products 2

8. References

S43

9. NMR-Spectra of 1, Catalysts and 2 


\section{General Remarks}

Reagents: Commercially available reagents and solvents were of reagent grade quality without any further purification.

Chromatography: Flash column chromatography was performed using silicycle silica gel (200-300 mesh).

Analytical thin-layer chromatography (TLC) was performed on $0.2 \mathrm{~mm}$ coated silica gel plates (HSGF 254) and visualized using a UV lamp (254 nm or $365 \mathrm{~nm}$ ).

Nuclear Magnetic Resonance Spectroscopy: ${ }^{1} \mathrm{H}$ NMR and ${ }^{13} \mathrm{C}$ NMR were recorded on magnet system 400'54 ascend purchased from Bruker Biospin AG.

${ }^{1} \mathrm{H}$ NMR spectra chemical shifts $(\delta)$ were reported in parts per million (ppm) referenced to hydrogen resonances in the NMR solvent (DMSO- $d_{6}$ ).

${ }^{13} \mathrm{C}$ NMR spectra chemical shifts $(\delta)$ were reported in parts per million (ppm) referenced to carbon resonances in the NMR solvent (DMSO- $\left.d_{6}\right)$.

ESI-MS spectra were recorded on Agilent Q-TOF 6520.

All electrochemical heteroaryl migration was carried out in an undivided electrochemical cell equipped with a carbon cloth anode $(40 \mathrm{~mm} \times 20 \mathrm{~mm})$ and a platinum plate cathode $(20 \mathrm{~mm}$ x $20 \mathrm{~mm})$. Electrolysis was conducted under an AXIOMET AX3003P potentiostat in constant current mode. Cyclic voltammogram experiments were investigated using a Metrohm Autolab PGSTAT204 workstation and Nova 2.0 software. 


\section{Synthesis of the Starting Materials and Catalysts}

\subsection{Synthesis of the Starting Materials}

1a-1t:

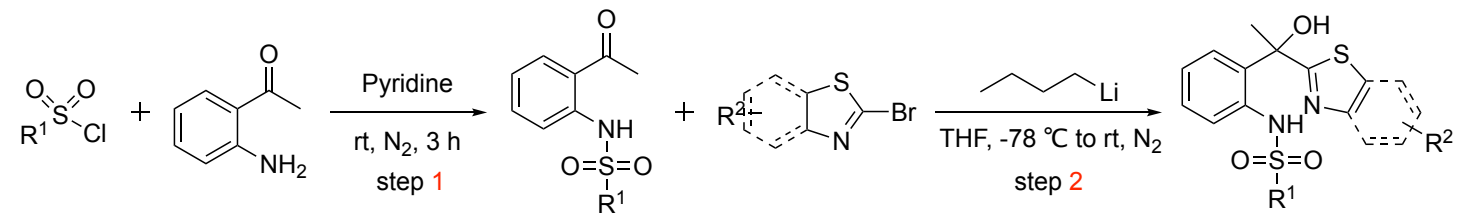

Step $1^{[1]}$ :

2-Aminoacetophenone (1 eq, $20 \mathrm{mmol}, 2.7014 \mathrm{~g}$ ) and the corresponding sulfonyl chloride (1.05 eq, $21 \mathrm{mmol})$ were dissolved in anhydrous pyridine $(15 \mathrm{~mL})$ under $\mathrm{N}_{2}$. The reaction mixture was stirred for $3 \mathrm{~h}$ at ambient temperature. The mixture was diluted with $\mathrm{CH}_{2} \mathrm{Cl}_{2}(150 \mathrm{~mL})$, which was washed with $\mathrm{HCl}$ solution $(3 \%, 150 \mathrm{~mL} \times$ 2) and water $(150 \mathrm{~mL})$. The separated organic layer was dried over anhydrous $\mathrm{Na}_{2} \mathrm{SO}_{4}$ and filtered. The filtrate was concentrated under reduced pressure to give the crude product, which was purified by column chromatographic on silica gel (200-300 mesh, eluent: petroleum ether/ethyl acetate 20:1)

\section{Step $2^{[2]}$ :}

To a stirred solution of the corresponding brominated heterocycle (15 mmol, $1.5 \mathrm{eq})$ in anhydrous THF (35 mL) was added $n$-butyllithium (15 mmol, $6 \mathrm{~mL}, 2.5 \mathrm{M}, 1.5 \mathrm{eq})$ dropwise at $-78^{\circ} \mathrm{C}$ under $\mathrm{N}_{2}$. After stirred for $0.5 \mathrm{~h}$, the solution of sulfonamide (10 mmol, 1 eq) in anhydrous THF $(30 \mathrm{~mL})$ was added dropwise to the mixture. The mixture was stirred for another $2 \mathrm{~h}$ at $0^{\circ} \mathrm{C}$ and quenched with saturated aq. $\mathrm{NH}_{4} \mathrm{Cl}$ 
(100 mL). The aqueous layer was extracted with EA $(120 \mathrm{~mL})$ for 2 times. The separated organic layer was dried over anhydrous $\mathrm{Na}_{2} \mathrm{SO}_{4}$ and filtered. The filtrate was concentrated under reduced pressure to give the crude product, which was purified by column chromatographic on silica gel (200-300 mesh).

1u-1w:

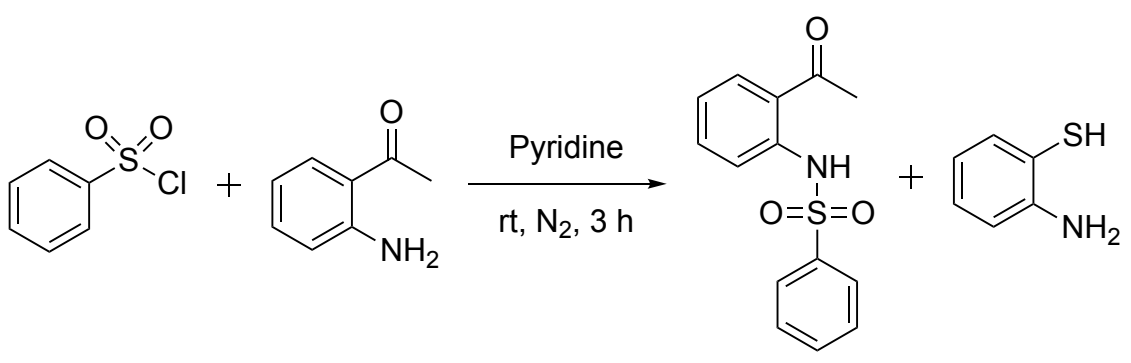<smiles>[R11]Nc1ccccc1C([R1])(O)c1nc2ccccc2s1</smiles>

Step $3^{[3]}$ :

$N$-(2-acetylphenyl)benzenesulfonamide (10 mmol, $2.7506 \mathrm{~g}, 1 \mathrm{eq})$, 2-aminobenzenethiol (12 mmol, $1.5004 \mathrm{~g}, 1.2 \mathrm{eq})$ and iodine (15 mmol, $3.8072 \mathrm{~g}, 1.5 \mathrm{eq})$ were dissolved in DMSO $(15 \mathrm{~mL})$. The mixture was stirred for $1 \mathrm{~h}$ at $100{ }^{\circ} \mathrm{C}$ (temperature of the oil bath) and quenched with saturated aq. $\mathrm{Na}_{2} \mathrm{SO}_{3}(150 \mathrm{~mL})$. The aqueous layer was extracted with EA $(150 \mathrm{~mL})$ for 2 times. The separated organic layer was dried over anhydrous $\mathrm{Na}_{2} \mathrm{SO}_{4}$ and filtered. The filtrate was concentrated under reduced pressure to give the crude product, which was purified by column chromatographic on silica gel (200-300 mesh, eluent: petroleum ether/ethyl acetate 18:1). 
Step $4^{[4]}$ :

To a stirred solution of the $N$-(2-(benzo[ $d]$ thiazole-2-carbonyl)phenyl)benzenesulfon amide (3 mmol, $1.1821 \mathrm{~g}, 1.0 \mathrm{eq})$ in anhydrous THF $(15 \mathrm{~mL})$ was added dropwise the corresponding Grignard reagent $(3.3 \mathrm{mmol}, 1.1 \mathrm{eq})$ at $-20^{\circ} \mathrm{C}$. The mixture was stirred for $2 \mathrm{~h}$ and quenched with saturated aq. $\mathrm{NH}_{4} \mathrm{Cl}(100 \mathrm{~mL})$. The aqueous layer was extracted with EA (120 mL) for 2 times. The separated organic layer was dried over anhydrous $\mathrm{Na}_{2} \mathrm{SO}_{4}$ and filtered. The filtrate was concentrated under reduced pressure to give the crude product, which was purified by column chromatographic on silica gel (200-300 mesh).

\subsection{Synthesis of the Catalysts ${ }^{[5]}$}

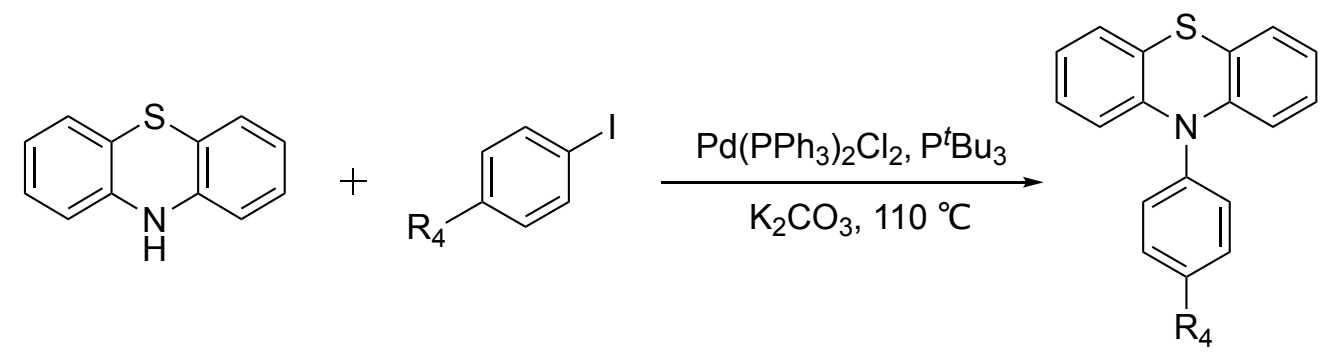

The corresponding iodine benzene ( $3 \mathrm{mmol}, 1 \mathrm{eq})$, phenothiazine (1.1 eq, $3.3 \mathrm{mmol}$, $656.9 \mathrm{mg}), \mathrm{K}_{2} \mathrm{CO}_{3}(3 \mathrm{eq}, 9 \mathrm{mmol}, 1243.9 \mathrm{mg}), \mathrm{Pd}\left(\mathrm{PPh}_{3}\right)_{2} \mathrm{Cl}_{2}(0.03 \mathrm{eq}, 0.09 \mathrm{mmol}$, $63.2 \mathrm{mg})$ and $\mathrm{P}^{t} \mathrm{Bu}_{3}(0.11 \mathrm{mmol}, 66.8 \mathrm{mg})$ were added to a dry Schlenk tube. The flask was evacuated and backfilled with pure $\mathrm{N}_{2}$ for 3 times. Then anhydrous toluene $(10 \mathrm{~mL})$ was added. The mixture was stirred at $110^{\circ} \mathrm{C}$ (temperature of the oil bath) for $24 \mathrm{~h}$ and quenched with saturated aq. $\mathrm{NH}_{4} \mathrm{Cl}(150 \mathrm{~mL})$. The aqueous layer was extracted with EA $(150 \mathrm{~mL})$. The separated organic layer was dried over anhydrous 
$\mathrm{Na}_{2} \mathrm{SO}_{4}$ and filtered. The filtrate was concentrated under reduced pressure to give the crude product, which was purified by column chromatographic on silica gel (200-300 mesh).

\section{General Procedure for the Electrochemical Heteroaryl Migration}

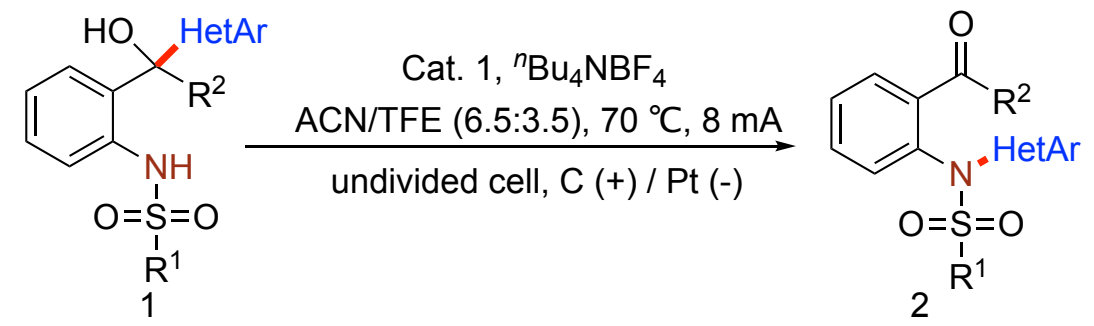

In an undivided cell equipped with a carbon cloth anode (40 $\mathrm{mm} \times 20 \mathrm{~mm})$ and a platinum plate cathode $(20 \mathrm{~mm} \times 20 \mathrm{~mm}), 1(0.3 \mathrm{mmol})$, Cat. $1(0.03 \mathrm{mmol}, 8.2 \mathrm{mg}$, $10 \mathrm{~mol} \%$ ) and ${ }^{n} \mathrm{Bu}_{4} \mathrm{NBF}_{4}$ (1 eq, $0.3 \mathrm{mmol}, 98.8 \mathrm{mg}$ ) were dissolved in a mixed solvent of ACN/TFE $[(6.5: 3.5) \mathrm{mL}]$. At $70^{\circ} \mathrm{C}$ (temperature of the oil bath), the reaction was started at a constant current of $8 \mathrm{~mA}$. After the reaction was complete (monitored by TLC), the mixture was concentrated under reduced pressure to give the crude product, which was purified by column chromatographic on silica gel (200-300 mesh). 


\section{Gram-Scale Synthesis of $2 b$}

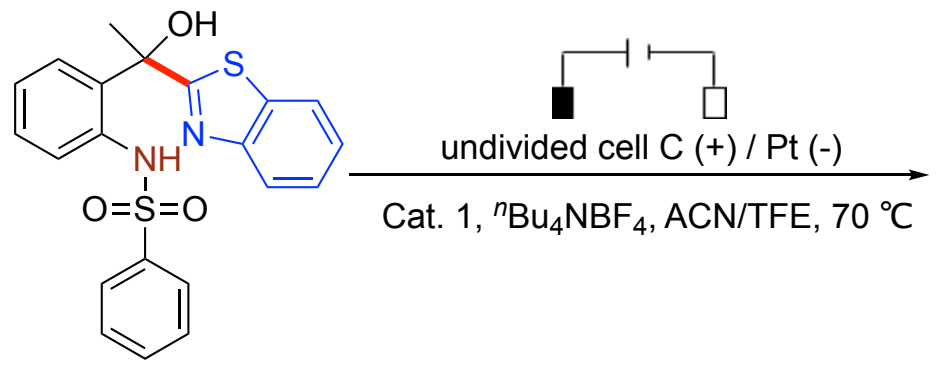

$1 \mathrm{~b}, 3 \mathrm{mmol}, 1.23 \mathrm{~g}$

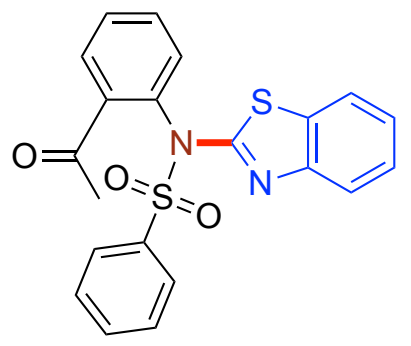

$2 \mathrm{~b}, 0.99 \mathrm{~g}, 81 \%$

In an undivided cell equipped with a carbon cloth anode (40 $\mathrm{mm} \times 20 \mathrm{~mm})$ and a platinum plate cathode $(20 \mathrm{~mm} \times 20 \mathrm{~mm}), 1 \mathrm{~b}(3 \mathrm{mmol}, 1.230 \mathrm{~g})$, Cat. 1 (0.3 mmol, $82.5 \mathrm{mg}, 10 \mathrm{~mol} \%)$ and ${ }^{n} \mathrm{Bu}_{4} \mathrm{NBF}_{4}(1 \mathrm{eq}, 3 \mathrm{mmol}, 0.988 \mathrm{~g})$ were dissolved in a mixed solvent of ACN/TFE [(39:21) $\mathrm{mL}]$. At $70^{\circ} \mathrm{C}$ (temperature of the oil bath), the reaction was started at a constant current of $16 \mathrm{~mA}$ for $10 \mathrm{~h}$. The mixture was concentrated under reduced pressure to give the crude product, which was purified by column chromatographic on silica gel (200-300 mesh, eluent: petroleum ether/ethyl acetate 18:1, yellow solid, $0.99 \mathrm{~g}, 81 \%$ ).

\section{Mechanistic Studies}

\subsection{Radical-trapping experiment}



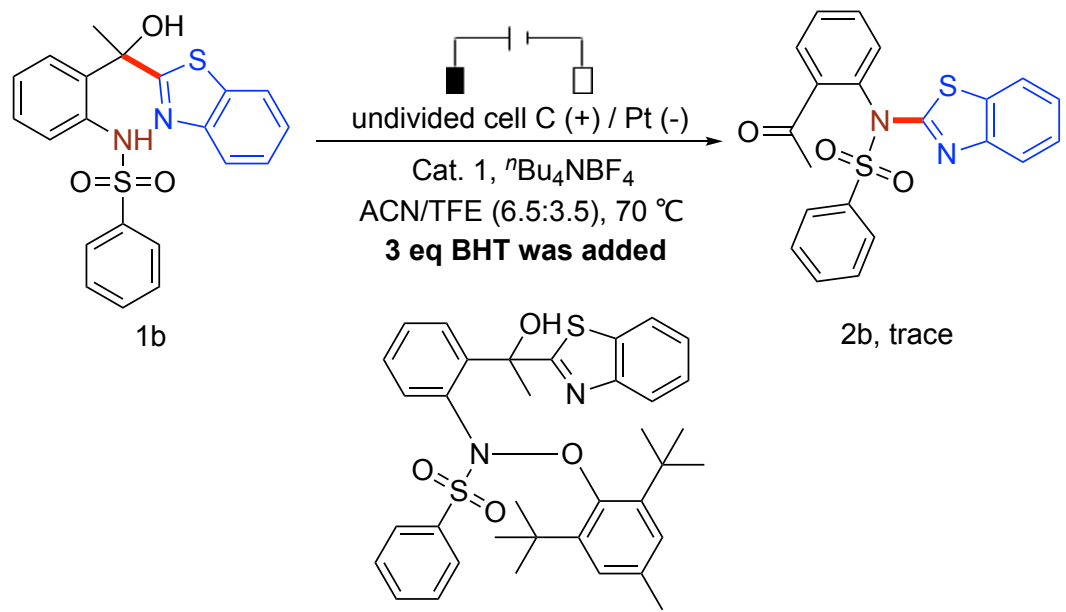

$1 \mathrm{~b}$

$2 b$, trace

Intermediate S-1

detected by MS (ESI)

In an undivided cell equipped with a carbon cloth anode (40 $\mathrm{mm} \times 20 \mathrm{~mm})$ and a platinum plate cathode $(20 \mathrm{~mm} \times 20 \mathrm{~mm}), 1(0.3 \mathrm{mmol})$, Cat. $1(0.03 \mathrm{mmol}, 8.2 \mathrm{mg}$, $10 \mathrm{~mol} \mathrm{\%}),{ }^{n} \mathrm{Bu}_{4} \mathrm{NBF}_{4}$ (1 eq, $0.3 \mathrm{mmol}, 98.8 \mathrm{mg}$ ) and BHT (3 eq, $0.9 \mathrm{mmol}, 198.3 \mathrm{mg}$ ) were dissolved in a mixed solvent of ACN/TFE $[(6.5: 3.5) \mathrm{mL}]$. At $70^{\circ} \mathrm{C}$ (temperature of the oil bath), the reaction was started at a constant current of $8 \mathrm{~mA}$ for $2 \mathrm{~h}$. Only trace amount of the desired product was detected by TLC. And, the corresponding radical-trapping intermediate $\mathbf{S - 1}$ was detected by MS-ESI (Calcd for $\mathrm{C}_{36} \mathrm{H}_{41} \mathrm{~N}_{2} \mathrm{O}_{4} \mathrm{~S}_{2}$ $[\mathrm{M}+\mathrm{H}]^{+}:$629.2502; found: $629.2523 . \mathrm{C}_{36} \mathrm{H}_{40} \mathrm{~N}_{2} \mathrm{O}_{4} \mathrm{~S}_{2} \mathrm{Na}[\mathrm{M}+\mathrm{Na}]^{+}: 651.2322$; found: 651.2345. Fig. S1).

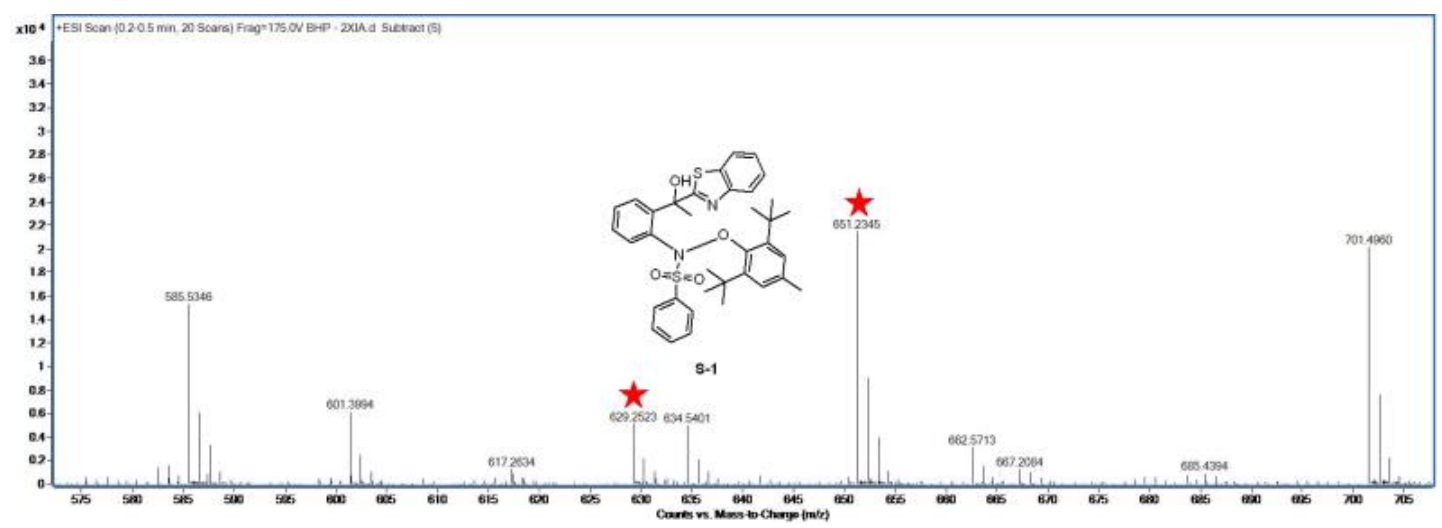

Fig. S1 HR-MS (ESI) analysis of S-1 


\subsection{Cyclic voltammetry experiments}

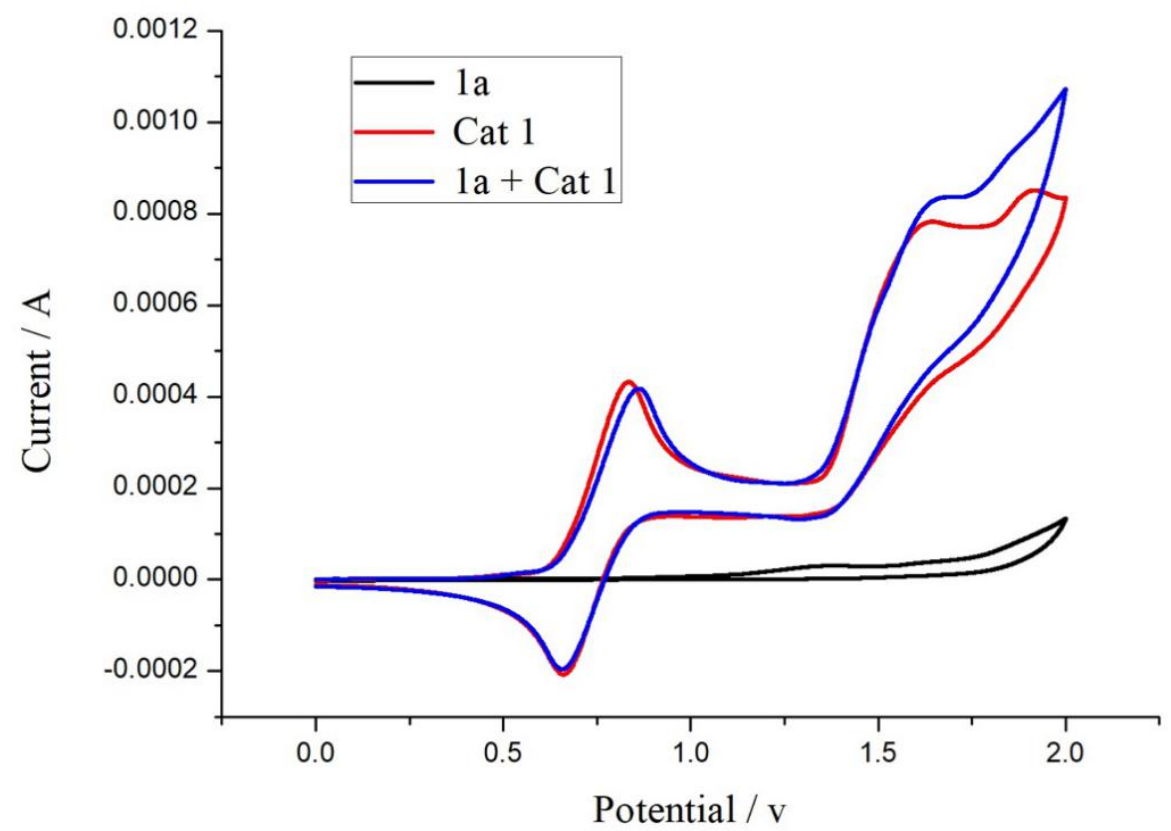

Fig. S2 Cyclic Voltammetry

$100 \mathrm{mVs}-1: n-\mathrm{Bu}_{4} \mathrm{NBF}_{4}(0.1 \mathrm{M}$ in $\mathrm{ACN}, 10 \mathrm{~mL})$

(black) substrate 1a $(0.1 \mathrm{mmol})$; (red) Cat. $1(0.1 \mathrm{mmol})$; (blue) $1 \mathrm{a}(0.1 \mathrm{mmol})$ and Cat. $1(0.1$ mmol).

The undivided cell was equipped with glassy-carbon disk working electrode (diameter, $3.0 \mathrm{~mm}$ ) and $\mathrm{Pt}$ wire auxiliary electrode. The $\mathrm{Ag} / \mathrm{AgCl}$ was used as reference electrode. The scan range was $0.0 \mathrm{~V}$ to $2.0 \mathrm{~V}$. The scan rate was $100 \mathrm{mVs}^{-1}$. 
6 Structure of $2 \mathrm{f}$ by X-Ray Crystallographic (CCDC: 1955548)

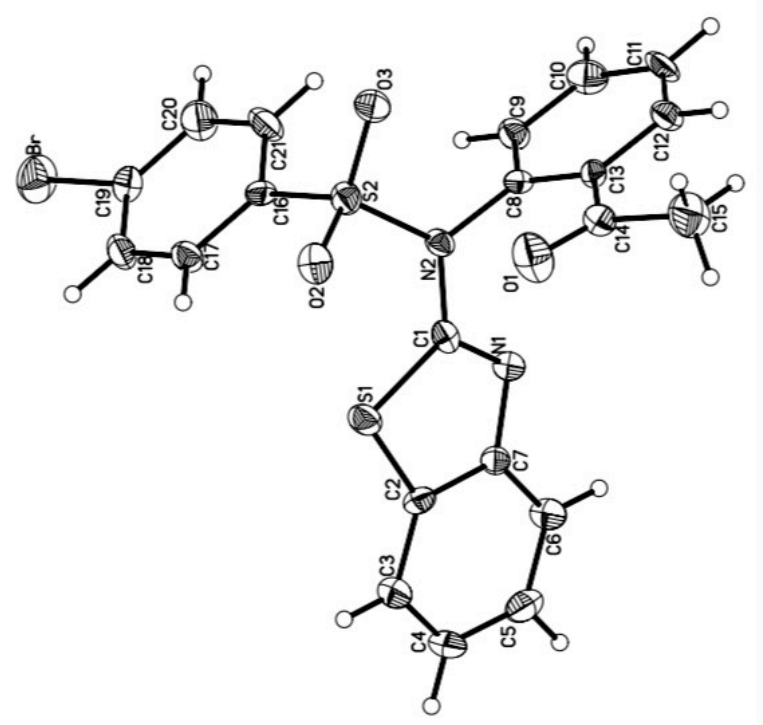

Figure S3 Structure of 2f by X-Ray Crystallographic (CCDC 1955548) 


\section{Characterization Data of and Starting Materials 1, Catalysts and Products 2}

7.1 Characterization Data of Starting Materials 1<smiles>Cc1ccc(S(=O)(=O)Nc2ccccc2C(C)(O)c2nc3ccccc3s2)cc1</smiles>

N-(2-(1-(benzo[d] thiazol-2-yl)-1-hydroxyethyl)phenyl)-4-methylbenzenesulfonam ide (1a):

White solid; Eluent: petroleum ether/ethyl acetate/dichloromethane 20:1:3; $2629.4 \mathrm{mg}$, 62\%; ${ }^{1}$ H NMR $\left(400 \mathrm{MHz}, \mathrm{DMSO}-d_{6}\right) \delta 9.97(\mathrm{~s}, 1 \mathrm{H}), 8.13-8.08(\mathrm{~m}, 2 \mathrm{H}), 7.83-7.78$ (m, 1H), $7.53-7.43(\mathrm{~m}, 4 \mathrm{H}), 7.31-7.23(\mathrm{~m}, 3 \mathrm{H}), 7.06(\mathrm{td}, J=7.6,1.3 \mathrm{~Hz}, 1 \mathrm{H}), 6.83$ - 6.79 (m, 2H), 2.14 (s, 3H), 1.94 (s, 3H); ${ }^{13}$ C NMR (101 MHz, DMSO-d $) \delta 179.0$, $152.9,143.4,136.0,135.9,134.8,132.5,129.3,128.9,127.7,126.4,126.1,125.2$, 123.3, 122.7, 122.3, 118.1, 77.1, 29.9, 20.9; HRMS (ESI-TOF) Calcd for $\mathrm{C}_{22} \mathrm{H}_{21} \mathrm{~N}_{2} \mathrm{O}_{3} \mathrm{~S}_{2}[\mathrm{M}+\mathrm{H}]^{+}$: 425.0988 ; found: 425.1001 .<smiles>CC(O)(c1nc2ccccc2s1)c1ccccc1NS(=O)(=O)c1ccccc1</smiles>

$N$-(2-(1-(benzo[d]thiazol-2-yl)-1-hydroxyethyl)phenyl)benzenesulfonamide (1b): 
White solid; Eluent: petroleum ether/ethyl acetate/dichloromethane 20:1:3; 2624.5 mg, 64\%; ${ }^{1}$ H NMR (400 MHz, DMSO-d $\left.d_{6}\right) 10.08(\mathrm{~s}, 1 \mathrm{H}), 8.15-8.09(\mathrm{~m}, 2 \mathrm{H}), 7.84(\mathrm{dd}$, $J=8.0,1.3 \mathrm{~Hz}, 1 \mathrm{H}), 7.50(\mathrm{td}, J=7.6,1.5 \mathrm{~Hz}, 1 \mathrm{H}), 7.48-7.42(\mathrm{~m}, 5 \mathrm{H}), 7.37(\mathrm{tt}, J=$ 7.2, $1.6 \mathrm{~Hz}, 1 \mathrm{H}), 7.25(\mathrm{td}, J=7.8,1.5 \mathrm{~Hz}, 1 \mathrm{H}), 7.14-7.02(\mathrm{~m}, 3 \mathrm{H}), 1.94(\mathrm{~s}, 3 \mathrm{H}) ;{ }^{13} \mathrm{C}$ NMR $\left(101 \mathrm{MHz}, \mathrm{DMSO}-d_{6}\right) \delta 179.0,152.7,138.8,135.8,134.8,133.0,132.5,128.9$, $128.8,127.7,126.5,126.2,125.2,123.4,122.8,122.4,118.2,77.2,29.8$; HRMS (ESI-TOF) Calcd for $\mathrm{C}_{21} \mathrm{H}_{19} \mathrm{~N}_{2} \mathrm{O}_{3} \mathrm{~S}_{2}[\mathrm{M}+\mathrm{H}]^{+}:$411.0832; found: 411.0842 .<smiles>CC(C)(C)c1ccc(S(=O)(=O)Nc2ccccc2C(C)(O)c2nc3ccccc3s2)cc1</smiles>

$N-(2-(1-(b e n z o[d]$ thiazol-2-yl)-1-hydroxyethyl)phenyl)-4-(tert-butyl)benzenesulfo namide (1c):

White solid; Eluent: petroleum ether/ethyl acetate/dichloromethane 20:1:3; $2843.4 \mathrm{mg}$, 61\%; ${ }^{1}$ H NMR (400 MHz, DMSO-d $\left.d_{6}\right) \delta 10.02(\mathrm{~s}, 1 \mathrm{H}), 8.18-8.14(\mathrm{~m}, 1 \mathrm{H}), 8.12(\mathrm{~s}$, 1H), $7.87-7.81(\mathrm{~m}, 1 \mathrm{H}), 7.54-7.44(\mathrm{~m}, 4 \mathrm{H}), 7.36-7.30(\mathrm{~m}, 2 \mathrm{H}), 7.25(\mathrm{ddd}, J=8.3$, 7.4, $1.5 \mathrm{~Hz}, 1 \mathrm{H}), 7.09-7.02(\mathrm{~m}, 3 \mathrm{H}), 1.95(\mathrm{~s}, 3 \mathrm{H}), 1.13(\mathrm{~s}, 9 \mathrm{H}) ;{ }^{13} \mathbf{C}$ NMR $(101 \mathrm{MHz}$ DMSO- $\left.d_{6}\right) \delta 179.0,156.0,152.8,136.0,135.9,134.8,132.3,129.0,127.7,126.5$, $126.2,125.7,125.1,123.1,122.7,122.4,117.6,77.1,34.7,30.6,29.7$; HRMS (ESI-TOF) Calcd for $\mathrm{C}_{25} \mathrm{H}_{27} \mathrm{~N}_{2} \mathrm{O}_{3} \mathrm{~S}_{2}[\mathrm{M}+\mathrm{H}]^{+}$: 467.1458; found: 467.1468. 
<smiles>CC(O)(c1nc2ccccc2s1)c1ccccc1NS(=O)(=O)c1ccc(F)cc1</smiles>

$N$-(2-(1-(benzo[d] thiazol-2-yl)-1-hydroxyethyl)phenyl)-4-fluorobenzenesulfonami de (1d):

White solid; Eluent: petroleum ether/ethyl acetate/dichloromethane 20:1:3; $2868.1 \mathrm{mg}$, 67\%; ${ }^{1}$ H NMR $\left(400 \mathrm{MHz}, \mathrm{DMSO}-d_{6}\right) \delta 10.02(\mathrm{~s}, 1 \mathrm{H}), 8.13(\mathrm{~s}, 1 \mathrm{H}), 8.11-8.07(\mathrm{~m}$, 1H), $7.81-7.77(\mathrm{~m}, 1 \mathrm{H}), 7.54(\mathrm{dd}, J=8.2,1.3 \mathrm{~Hz}, 1 \mathrm{H}), 7.51-7.43(\mathrm{~m}, 5 \mathrm{H}), 7.30(\mathrm{td}$, $J=7.2,1.5 \mathrm{~Hz}, 1 \mathrm{H}), 7.10(\mathrm{td}, J=7.6,1.3 \mathrm{~Hz}, 1 \mathrm{H}), 6.87-6.80(\mathrm{~m}, 2 \mathrm{H}), 1.94(\mathrm{~s}, 3 \mathrm{H})$; ${ }^{13}$ C NMR (101 MHz, DMSO-d $) \delta 178.9,165.4,162.9,152.7,135.7,135.3,135.2$, $134.7,132.9,129.3,129.2,129.0,127.8,126.2,125.1,123.7,122.6,122.3,118.5$, 116.0, 115.8, 77.1, 29.9; ${ }^{19}$ F NMR (376 MHz, DMSO- $\left.d_{6}\right) \delta-105.2$; HRMS (ESI-TOF) Calcd for $\mathrm{C}_{21} \mathrm{H}_{18} \mathrm{~N}_{2} \mathrm{O}_{3} \mathrm{~S}_{2} \mathrm{~F}[\mathrm{M}+\mathrm{H}]^{+}:$429.0737; found: 429.0744 .<smiles>CC(O)(c1nc2ccccc2s1)c1ccccc1NS(=O)(=O)c1ccc(Cl)cc1</smiles>

$N$-(2-(1-(benzo[d] thiazol-2-yl)-1-hydroxyethyl)phenyl)-4-chlorobenzenesulfonami de (1e): 
Yellow solid; Eluent: petroleum ether/ethyl acetate/dichloromethane 20:1:3; 2930.7 mg, 66\%; ${ }^{1}$ H NMR (400 MHz, DMSO- $\left.d_{6}\right) \delta 10.03(\mathrm{~s}, 1 \mathrm{H}), 8.14-8.08(\mathrm{~m}, 2 \mathrm{H}), 7.80$ $-7.75(\mathrm{~m}, 1 \mathrm{H}), 7.55-7.45(\mathrm{~m}, 4 \mathrm{H}), 7.39(\mathrm{dt}, J=8.7,20 \mathrm{~Hz}, 2 \mathrm{H}), 7.30(\mathrm{td}, J=7.2$, $1.2 \mathrm{~Hz}, 1 \mathrm{H}), 7.11(\mathrm{td}, J=7.6,1.3 \mathrm{~Hz}, 1 \mathrm{H}), 7.05(\mathrm{dt}, J=8.7,1.9 \mathrm{~Hz}, 2 \mathrm{H}), 1.93(\mathrm{~s}, 3 \mathrm{H})$;

${ }^{13}$ C NMR (101 MHz, DMSO- $\left.d_{6}\right) \delta 179.8,152.9,138.9,137.5,137.3,134.8,133.1$, $128.8,128.7,128.0,127.5,126.1,125.0,122.62,122.55,122.3,118.5,77.2,29.9$; HRMS (ESI-TOF) Calcd for $\mathrm{C}_{21} \mathrm{H}_{18} \mathrm{~N}_{2} \mathrm{O}_{3} \mathrm{~S}_{2} \mathrm{Cl}[\mathrm{M}+\mathrm{H}]^{+}$: 445.0442 ; found: 445.0441.<smiles>CC(O)(c1nc2ccccc2s1)c1ccccc1NS(=O)(=O)c1ccc(Br)cc1</smiles>

$N$-(2-(1-(benzo[d] thiazol-2-yl)-1-hydroxyethyl)phenyl)-4-bromobenzenesulfonami de (1f):

Yellow solid; Eluent: petroleum ether/ethyl acetate/dichloromethane 20:1:3; 3220.7 mg, 66\%; ${ }^{1}$ H NMR (400 MHz, DMSO- $\left.d_{6}\right) \delta 10.04(\mathrm{~s}, 1 \mathrm{H}), 8.18-8.05(\mathrm{~m}, 2 \mathrm{H}), 7.80$ $-7.76(\mathrm{~m}, 1 \mathrm{H}), 7.56-7.43(\mathrm{~m}, 4 \mathrm{H}), 7.35-7.26(\mathrm{~m}, 3 \mathrm{H}), 7.19(\mathrm{dt}, J=8.7,1.9 \mathrm{~Hz}$, 2H), $7.11(\mathrm{td}, J=7.7,1.3 \mathrm{~Hz}, 1 \mathrm{H}), 1.94(\mathrm{~s}, 3 \mathrm{H}) ;{ }^{13} \mathbf{C}$ NMR $\left(101 \mathrm{MHz}, \mathrm{DMSO}-d_{6}\right) \delta$ $179.2,152.8,138.4,136.2,134.7,133.0,131.8,129.0,128.1,127.7,126.8,126.3$, 125.1, 123.4, 122.6, 122.3, 118.5, 77.1, 29.9; HRMS (ESI-TOF) Calcd for $\mathrm{C}_{21} \mathrm{H}_{18} \mathrm{~N}_{2} \mathrm{O}_{3} \mathrm{~S}_{2} \mathrm{Br}[\mathrm{M}+\mathrm{H}]^{+}:$488.9937; found: 488.9942. 
<smiles>CC(O)(c1nc2ccccc2s1)c1ccccc1NS(=O)(=O)c1ccc(I)cc1</smiles>

$N$-(2-(1-(benzo[d] thiazol-2-yl)-1-hydroxyethyl)phenyl)-4-iodobenzenesulfonamid (1g):

Yellow solid; Eluent: petroleum ether/ethyl acetate/dichloromethane 20:1:3; 3108.6 mg, 58\%; ${ }^{1}$ H NMR (400 MHz, DMSO-d $\left.d_{6}\right) \delta 10.02(\mathrm{~s}, 1 \mathrm{H}), 8.16-8.09(\mathrm{~m}, 2 \mathrm{H}), 7.80$ $-7.76(\mathrm{~m}, 1 \mathrm{H}), 7.55-7.44(\mathrm{~m}, 4 \mathrm{H}), 7.34(\mathrm{dt}, J=8.8,1.9 \mathrm{~Hz}, 2 \mathrm{H}), 7.32-7.25(\mathrm{~m}$, 1H), $7.16-7.07(\mathrm{~m}, 3 \mathrm{H}), 1.94(\mathrm{~s}, 3 \mathrm{H}) ;{ }^{13} \mathrm{C}$ NMR (101 MHz, DMSO-d $)_{6} \delta 178.8$, $152.8,138.3,137.7,135.6,134.7,132.8,129.1,127.8,126.3,125.2,123.7,122.6$ 122.3, 118.3, 101.6, 77.1, 29.9; HRMS (ESI-TOF) Calcd for $\mathrm{C}_{21} \mathrm{H}_{18} \mathrm{~N}_{2} \mathrm{O}_{3} \mathrm{~S}_{2} \mathrm{I}[\mathrm{M}+\mathrm{H}]^{+}$: 536.9798; found: 536.9797 .<smiles>CC(O)(c1nc2ccccc2s1)c1ccccc1NS(=O)(=O)c1ccc(-c2ccccc2)cc1</smiles>

$N$-(2-(1-(benzo[d]thiazol-2-yl)-1-hydroxyethyl)phenyl)-[1,1'-biphenyl]-4-sulfona mide (1h):

Yellow solid; Eluent: petroleum ether/ethyl acetate/dichloromethane 20:1:3; 2916.7 mg, 60\%; ${ }^{1}$ H NMR (400 MHz, DMSO- $d_{6}$ ) $\delta 10.10(\mathrm{~s}, 1 \mathrm{H}), 8.17(\mathrm{~s}, 1 \mathrm{H}), 8.13-8.08$ 
(m, 1H), $7.82-7.77(\mathrm{~m}, 1 \mathrm{H}), 7.56-7.45(\mathrm{~m}, 8 \mathrm{H}), 7.45-7.37(\mathrm{~m}, 3 \mathrm{H}), 7.37-7.26$ (m, 3H), 7.09 (td, $J=7.7,1.3 \mathrm{~Hz}, 1 \mathrm{H}), 1.97(\mathrm{~s}, 3 \mathrm{H}) ;{ }^{13} \mathbf{C}$ NMR (101 MHz, DMSO-d $\left.{ }_{6}\right)$ $\delta 179.0,152.8,144.3,138.0,137.5,135.8,134.7,132.6,129.01,128.98,128.6,127.7$, $127.1,127.0,126.9,126.1,125.1,123.4,122.6,122.3,118.1,77.1,29.8$; HRMS (ESI-TOF) Calcd for $\mathrm{C}_{27} \mathrm{H}_{23} \mathrm{~N}_{2} \mathrm{O}_{3} \mathrm{~S}_{2}[\mathrm{M}+\mathrm{H}]^{+}$: 487.1145 ; found: 487.1157.<smiles>CC(O)(c1nc2ccccc2s1)c1ccccc1NS(=O)(=O)c1ccc([N+](=O)[O-])cc1</smiles>

$N$-(2-(1-(benzo[d] thiazol-2-yl)-1-hydroxyethyl)phenyl)-4-nitrobenzenesulfonamid e (1i):

Brown solid; Eluent: petroleum ether/ethyl acetate/dichloromethane 20:1:5, 2684.9 mg, 59\%; ${ }^{1}$ H NMR (400 MHz, DMSO- $\left.d_{6}\right) \delta 10.11(\mathrm{~s}, 1 \mathrm{H}), 8.19(\mathrm{~s}, 1 \mathrm{H}), 8.04-7.99$ (m, 1H), $7.77-7.72(\mathrm{~m}, 2 \mathrm{H}), 7.68(\mathrm{dd}, J=8.2,1.3 \mathrm{~Hz}, 1 \mathrm{H}), 7.64-7.60(\mathrm{~m}, 1 \mathrm{H}), 7.59$ $-7.53(\mathrm{~m}, 3 \mathrm{H}), 7.41-7.29(\mathrm{~m}, 3 \mathrm{H}), 7.20(\mathrm{td}, J=7.7,1.3 \mathrm{~Hz}, 1 \mathrm{H}), 1.92(\mathrm{~s}, 3 \mathrm{H}) ;{ }^{13} \mathrm{C}$ NMR (101 MHz, DMSO-d $\left.d_{6}\right) \delta 178.7,152.5,149.0,144.3,135.4,134.4,133.6,129.3$, $127.9,127.2,126.0,125.1,124.3,123.8,122.3,122.2,119.1,76.9,30.0$; HRMS (ESI-TOF) Calcd for $\mathrm{C}_{21} \mathrm{H}_{18} \mathrm{~N}_{3} \mathrm{O}_{5} \mathrm{~S}_{2}[\mathrm{M}+\mathrm{H}]^{+}$: 456.0682; found: 456.0685 . 
<smiles>CC(O)(c1nc2ccccc2s1)c1ccccc1NS(=O)(=O)c1ccccc1Cl</smiles>

$N$-(2-(1-(benzo[d] thiazol-2-yl)-1-hydroxyethyl)phenyl)-2-chlorobenzenesulfonami de (1j):

Yellow solid; Eluent: petroleum ether/ethyl acetate/dichloromethane 20:1:3; 2841.8 mg, 64\%; ${ }^{1}$ H NMR (400 MHz, DMSO- $\left.d_{6}\right) \delta 10.63(\mathrm{~s}, 1 \mathrm{H}), 8.16-8.11(\mathrm{~m}, 2 \mathrm{H}), 8.10$ $-8.06(\mathrm{~m}, 1 \mathrm{H}), 7.97-7.90(\mathrm{~m}, 1 \mathrm{H}), 7.67-7.58(\mathrm{~m}, 2 \mathrm{H}), 7.57-7.40(\mathrm{~m}, 4 \mathrm{H}), 7.21-$ $7.12(\mathrm{~m}, 1 \mathrm{H}), 7.07-7.01(\mathrm{~m}, 2 \mathrm{H}), 2.08(\mathrm{~s}, 3 \mathrm{H}) ;{ }^{13} \mathbf{C}$ NMR (101 MHz, DMSO-d $) \delta$ $180.0,153.0,137.8,135.9,135.28,135.26,132.9,132.4,131.4,131.2,129.2,128.5$ 128.1, 126.7, 125.7, 123.7, 123.1, 122.8, 118.1, 77.7, 29.9; HRMS (ESI-TOF) Calcd for $\mathrm{C}_{21} \mathrm{H}_{18} \mathrm{~N}_{2} \mathrm{O}_{3} \mathrm{~S}_{2} \mathrm{Cl}[\mathrm{M}+\mathrm{H}]^{+}$: 445.0442; found: 445.0448 .<smiles>CC(O)(c1nc2ccccc2s1)c1ccccc1NS(=O)(=O)c1cccc(Cl)c1</smiles>

$N$-(2-(1-(benzo[d] thiazol-2-yl)-1-hydroxyethyl)phenyl)-3-chlorobenzenesulfonami de (1k):

Yellow solid; Eluent: petroleum ether/ethyl acetate/dichloromethane 20:1:3; 2841.9 mg, 64\%; ${ }^{1}$ H NMR (400 MHz, DMSO-d $) \delta 10.23(\mathrm{~s}, 1 \mathrm{H}), 8.15(\mathrm{~s}, 1 \mathrm{H}), 8.07(\mathrm{dd}, J=$ 7.3, 1.2 Hz, 1H), $7.86-7.81(\mathrm{~m}, 1 \mathrm{H}), 7.56(\mathrm{t}, J=1.9 \mathrm{~Hz}, 1 \mathrm{H}), 7.52-7.36(\mathrm{~m}, 6 \mathrm{H})$, 
$7.29(\mathrm{td}, J=7.8,1.5 \mathrm{~Hz}, 1 \mathrm{H}), 7.15-7.07$ (m, 2H), $1.95(\mathrm{~s}, 3 \mathrm{H}) ;{ }^{13} \mathbf{C}$ NMR $(101 \mathrm{MHz}$ DMSO- $\left.d_{6}\right) \delta 178.8,152.6,140.9,135.4,134.7,133.6,132.93,132.88,130.8,129.0$, $127.8,126.2,126.0,125.2,124.9,123.9,122.7,122.3,118.7,77.2,29.8$; HRMS (ESI-TOF) Calcd for $\mathrm{C}_{21} \mathrm{H}_{18} \mathrm{~N}_{2} \mathrm{O}_{3} \mathrm{~S}_{2} \mathrm{Cl}[\mathrm{M}+\mathrm{H}]^{+}$: 445.0442; found: 445.0449.<smiles>Cc1cccc(S(=O)(=O)Nc2ccccc2C(C)(O)c2nc3ccccc3s2)c1</smiles>

$N$-(2-(1-(benzo[d] thiazol-2-yl)-1-hydroxyethyl)phenyl)-3-methylbenzenesulfonam ide (11):

Yellow solid; Eluent: petroleum ether/ethyl acetate/dichloromethane 20:1:3; 2544.5 mg, 60\%; ${ }^{1} \mathbf{H}$ NMR (400 MHz, DMSO- $\left.d_{6}\right) \delta 10.16(\mathrm{~s}, 1 \mathrm{H}), 8.15-8.05(\mathrm{~m}, 2 \mathrm{H}), 7.90$ - $7.84(\mathrm{~m}, 1 \mathrm{H}), 7.53-7.36(\mathrm{~m}, 5 \mathrm{H}), 7.32-7.17(\mathrm{~m}, 3 \mathrm{H}), 7.08-6.99(\mathrm{~m}, 2 \mathrm{H}), 2.08(\mathrm{~s}$, 3H), $1.96(\mathrm{~s}, 3 \mathrm{H}) ;{ }^{13} \mathbf{C}$ NMR (101 MHz, DMSO-d $\left.{ }_{6}\right) \delta$ 179.1, 152.7, 139.0, 138.9, $135.9,134.8,133.6,132.3,128.9,128.7,127.7,126.7,126.2,125.2,123.7,123.2$, 122.7, 122.4, 118.1, 77.2, 29.8, 20.6; HRMS (ESI-TOF) Calcd for $\mathrm{C}_{22} \mathrm{H}_{21} \mathrm{~N}_{2} \mathrm{O}_{3} \mathrm{~S}_{2}$ $[\mathrm{M}+\mathrm{H}]^{+}:$425.0988; found: 425.0993 .<smiles>CC(O)(c1nc2ccccc2s1)c1ccccc1NS(=O)(=O)c1cccnc1</smiles> 


\section{$N$-(2-(1-(benzo[d]thiazol-2-yl)-1-hydroxyethyl)phenyl)pyridine-3-sulfonamide}

(1m):

Brown solid; Eluent: petroleum ether/ethyl acetate/dichloromethane 20:1:5; 2096.5 mg, 51\%; ${ }^{1} \mathbf{H}$ NMR (400 MHz, DMSO-d $) \delta 10.25(\mathrm{~s}, 1 \mathrm{H}), 8.72(\mathrm{~d}, J=2.4 \mathrm{~Hz}, 1 \mathrm{H})$, $8.48(\mathrm{~d}, J=4.0 \mathrm{~Hz}, 1 \mathrm{H}), 8.17(\mathrm{~s}, 1 \mathrm{H}), 8.09-8.03(\mathrm{~m}, 1 \mathrm{H}), 7.83-7.78(\mathrm{~m}, 1 \mathrm{H}), 7.76$ $(\mathrm{ddd}, J=8.1,2.4,1.5 \mathrm{~Hz}, 1 \mathrm{H}), 7.55-7.41(\mathrm{~m}, 4 \mathrm{H}), 7.34-7.28(\mathrm{~m}, 1 \mathrm{H}), 7.12(\mathrm{td}, J=$ 7.6, $1.3 \mathrm{~Hz}, 1 \mathrm{H}), 7.04(\mathrm{dd}, J=8.0,4.8 \mathrm{~Hz}, 1 \mathrm{H}), 1.93(\mathrm{~s}, 3 \mathrm{H}) ;{ }^{13} \mathbf{C}$ NMR $(101 \mathrm{MHz}$, DMSO- $\left.d_{6}\right) \delta 178.8,153.3,152.6,146.7,135.5,135.3,134.7,134.0,133.1,129.1$, 127.9, 126.2, 125.2, 124.1, 123.7, 122.7, 122.4, 119.0, 77.2, 29.9; HRMS (ESI-TOF) Calcd for $\mathrm{C}_{20} \mathrm{H}_{18} \mathrm{~N}_{3} \mathrm{O}_{3} \mathrm{~S}_{2}[\mathrm{M}+\mathrm{H}]^{+}$: 412.0784; found: 412.0790 .

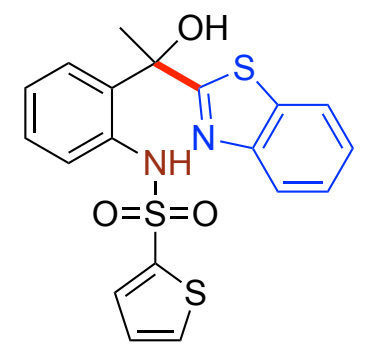

$N$-(2-(1-(benzo[d]thiazol-2-yl)-1-hydroxyethyl)phenyl)thiophene-2-sulfonamide (1n):

Brown solid; Eluent: petroleum ether/ethyl acetate/dichloromethane 20:1:3; 1830.5 mg, 44\%; ${ }^{1}$ H NMR (400 MHz, DMSO- $\left.d_{6}\right) \delta 10.38(\mathrm{~s}, 1 \mathrm{H}), 8.17(\mathrm{~s}, 1 \mathrm{H}), 8.10-8.05$ (m, 1H), $7.92-7.85(\mathrm{~m}, 1 \mathrm{H}), 7.64(\mathrm{dd}, J=5.0,1.4 \mathrm{~Hz}, 1 \mathrm{H}), 7.58(\mathrm{dd}, J=8.2,1.3 \mathrm{~Hz}$, 1H), $7.54-7.40(\mathrm{~m}, 3 \mathrm{H}), 7.35(\mathrm{dd}, J=3.8,1.4 \mathrm{~Hz}, 1 \mathrm{H}), 7.33-7.27(\mathrm{~m}, 1 \mathrm{H}), 7.10(\mathrm{td}$, $J=7.6,1.3 \mathrm{~Hz}, 1 \mathrm{H}), 6.76(\mathrm{dd}, J=5.0,3.8 \mathrm{~Hz}, 1 \mathrm{H}), 1.97(\mathrm{~s}, 3 \mathrm{H}),{ }^{13} \mathbf{C}$ NMR $(101 \mathrm{MHz}$, DMSO- $\left.d_{6}\right) \delta 179.1,152.7,139.3,135.6,134.9,133.4,132.9,132.5,128.9,127.7$ 
127.3, 126.2, 125.2, 123.8, 122.8, 122.3, 118.8, 77.3, 29.8; HRMS (ESI-TOF) Calcd for $\mathrm{C}_{19} \mathrm{H}_{17} \mathrm{~N}_{2} \mathrm{O}_{3} \mathrm{~S}_{3}[\mathrm{M}+\mathrm{H}]^{+}:$417.0396; found: 417.0402 .<smiles>Cc1cnc2c(S(=O)(=O)Nc3ccccc3C(C)(O)c3nc4ccccc4s3)cccc2c1</smiles>

$N$-(2-(1-(benzo[d]thiazol-2-yl)-1-hydroxyethyl)phenyl)-3-methylquinoline-8-sulfo namide (10):

Brown solid; Eluent: petroleum ether/ethyl acetate/dichloromethane 20:1:5; 2280.5 mg, 48\%; ${ }^{1} \mathbf{H}$ NMR (400 MHz, DMSO-d $) \delta 10.48(\mathrm{~s}, 1 \mathrm{H}), 8.75(\mathrm{~d}, J=2.2 \mathrm{~Hz}, 1 \mathrm{H})$, $8.36(\mathrm{dd}, J=7.3,1.4 \mathrm{~Hz}, 1 \mathrm{H}), 8.20(\mathrm{dd}, J=2.3,1.2 \mathrm{~Hz}, 1 \mathrm{H}), 8.14(\mathrm{dd}, J=8.3,1.4 \mathrm{~Hz}$ 1H), $8.07-8.03(\mathrm{~m}, 1 \mathrm{H}), 7.92-7.87(\mathrm{~m}, 1 \mathrm{H}), 7.75-7.65(\mathrm{~m}, 2 \mathrm{H}), 7.52-7.46(\mathrm{~m}$, 1H), $7.45-7.40(\mathrm{~m}, 1 \mathrm{H}), 7.33(\mathrm{dd}, J=8.0,1.5 \mathrm{~Hz}, 1 \mathrm{H}), 7.28(\mathrm{dd}, J=8.3,1.3 \mathrm{~Hz}, 1 \mathrm{H})$, $7.15-7.09(\mathrm{~m}, 1 \mathrm{H}), 6.94(\mathrm{td}, J=7.6,1.3 \mathrm{~Hz}, 1 \mathrm{H}), 2.46(\mathrm{~s}, J=1.0 \mathrm{~Hz}, 3 \mathrm{H}), 1.98(\mathrm{~s}$, $3 \mathrm{H}) ;{ }^{13} \mathbf{C}$ NMR (101 MHz, DMSO- $\left.d_{6}\right) \delta 179.4,153.4,152.6,140.8,136.5,136.0$, $135.1,134.9,133.4,132.6,132.1,129.9,128.4,128.3,127.4,126.0,125.6,125.0$, 122.7, 122.4, 122.2, 117.8, 76.9, 29.5, 18.2; HRMS (ESI-TOF) Calcd for $\mathrm{C}_{25} \mathrm{H}_{22} \mathrm{~N}_{3} \mathrm{O}_{3} \mathrm{~S}_{2}[\mathrm{M}+\mathrm{H}]^{+}:$476.1097; found: 476.1105.<smiles>CCS(=O)(=O)Nc1ccccc1C(C)(O)c1nc2ccccc2s1</smiles> 
N-(2-(1-(benzo[d]thiazol-2-yl)-1-hydroxyethyl)phenyl)ethanesulfonamide (1p):

White solid; Eluent: petroleum ether/ethyl acetate/dichloromethane 20:1:5; $2570.8 \mathrm{mg}$, 71\%; ${ }^{1}$ H NMR (400 MHz, DMSO- $\left.d_{6}\right) \delta 9.54(\mathrm{~s}, 1 \mathrm{H}), 8.10(\mathrm{ddd}, J=7.9,1.3,0.6 \mathrm{~Hz}$ 1H), $8.04(\mathrm{~s}, 1 \mathrm{H}), 7.92(\mathrm{ddd}, J=8.0,1.2,0.6 \mathrm{~Hz}, 1 \mathrm{H}), 7.54-7.46(\mathrm{~m}, 3 \mathrm{H}), 7.43$ (ddd, $J=8.4,7.2,1.3 \mathrm{~Hz}, 1 \mathrm{H}), 7.35-7.29(\mathrm{~m}, 1 \mathrm{H}), 7.11(\mathrm{td}, J=7.6,1.3 \mathrm{~Hz}, 1 \mathrm{H}), 2.91$ (hept, $J=7.2 \mathrm{~Hz}, 2 \mathrm{H}), 2.07$ (s, 3H), 0.89 (t, $J=7.3 \mathrm{~Hz}, 3 \mathrm{H}) ;{ }^{13} \mathbf{C}$ NMR (101 MHz, DMSO- $\left.d_{6}\right) \delta 179.3,152.6,136.5,134.7,132.3,129.1,127.7,126.3,125.3,123.0$ 122.6, 122.4, 118.3, 77.2, 46.0, 29.7, 7.6; HRMS (ESI-TOF) Calcd for $\mathrm{C}_{17} \mathrm{H}_{19} \mathrm{~N}_{2} \mathrm{O}_{3} \mathrm{~S}_{2}$ $[\mathrm{M}+\mathrm{H}]^{+}:$363.0832; found: 363.0838 .

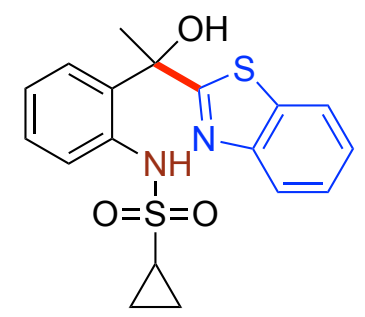

$N$-(2-(1-(benzo[d]thiazol-2-yl)-1-hydroxyethyl)phenyl)cyclopropanesulfonamide (1q):

White solid; Eluent: petroleum ether/ethyl acetate/dichloromethane 20:1:5; $2468.9 \mathrm{mg}$, $66 \% ;{ }^{1}$ H NMR $\left(400 \mathrm{MHz}, \mathrm{DMSO}-d_{6}\right) \delta 9.58(\mathrm{~s}, 1 \mathrm{H}), 8.13-8.08(\mathrm{~m}, 1 \mathrm{H}), 8.01(\mathrm{~s}$, 1H), $7.95-7.89(\mathrm{~m}, 1 \mathrm{H}), 7.58-7.46(\mathrm{~m}, 3 \mathrm{H}), 7.46-7.40(\mathrm{~m}, 1 \mathrm{H}), 7.36-7.30(\mathrm{~m}$, 1H), $7.13(\mathrm{td}, J=7.7,1.3 \mathrm{~Hz}, 1 \mathrm{H}), 2.27-2.18(\mathrm{~m}, 1 \mathrm{H}), 2.07(\mathrm{~s}, 3 \mathrm{H}), 0.86-0.77(\mathrm{~m}$, 1H), $0.76-0.64(\mathrm{~m}, 2 \mathrm{H}), 0.53-0.43(\mathrm{~m}, 1 \mathrm{H}) ;{ }^{13} \mathbf{C}$ NMR $\left(101 \mathrm{MHz}, \mathrm{DMSO}-d_{6}\right) \delta$ $179.5,152.7,136.4,134.8,133.0,128.9,127.6,126.3,125.3,123.3,122.6,122.4$ 
119.4, 77.1, 29.8, 29.7, 5.3, 4.8; HRMS (ESI-TOF) Calcd for $\mathrm{C}_{18} \mathrm{H}_{19} \mathrm{~N}_{2} \mathrm{O}_{3} \mathrm{~S}_{2}[\mathrm{M}+\mathrm{H}]^{+}$: 375.0832; found: 375.0838 .<smiles>COc1ccc2nc(C(C)(O)c3ccccc3NS(=O)(=O)c3ccccc3)sc2c1</smiles>

$N$-(2-(1-hydroxy-1-(6-methoxybenzo[d]thiazol-2-yl)ethyl)phenyl)benzenesulfona mide (1r):

White solid; Eluent: petroleum ether/ethyl acetate/dichloromethane 20:1:3; $2288.5 \mathrm{mg}$, 52\%; ${ }^{1} \mathbf{H}$ NMR $\left(400 \mathrm{MHz}, \mathrm{DMSO}-d_{6}\right) \delta 10.11(\mathrm{~s}, 1 \mathrm{H}), 8.04(\mathrm{~s}, 1 \mathrm{H}), 7.73(\mathrm{~d}, J=9.0$ $\mathrm{Hz}, 1 \mathrm{H}), 7.67(\mathrm{~d}, J=2.6 \mathrm{~Hz}, 1 \mathrm{H}), 7.52-7.48(\mathrm{~m}, 2 \mathrm{H}), 7.46-7.38(\mathrm{~m}, 3 \mathrm{H}), 7.26-$ $7.15(\mathrm{~m}, 3 \mathrm{H}), 7.09$ (dd, $J=9.0,2.6 \mathrm{~Hz}, 1 \mathrm{H}), 7.04(\mathrm{td}, J=7.7,1.3 \mathrm{~Hz}, 1 \mathrm{H}), 3.84(\mathrm{~s}$, 3H), $1.91(\mathrm{~s}, 3 \mathrm{H}) ;{ }^{13} \mathbf{C}$ NMR (101 MHz, DMSO-d $\left.d_{6}\right) \delta$ 176.1, 157.2, 147.0, 138.9, $136.3,135.7,133.0,132.6,128.9,128.8,127.6,126.5,123.3,123.3,118.2,115.5$, 104.8, 77.1, 55.8, 29.7; HRMS (ESI-TOF) Calcd for $\mathrm{C}_{22} \mathrm{H}_{21} \mathrm{~N}_{2} \mathrm{O}_{4} \mathrm{~S}_{2}[\mathrm{M}+\mathrm{H}]^{+}$: 441.0937; found: 441.0946 .<smiles>CC(O)(c1nccs1)c1ccccc1NS(=O)(=O)c1ccccc1</smiles>

N-(2-(1-hydroxy-1-(thiazol-2-yl)ethyl)phenyl)benzenesulfonamide (1s): 
White solid; Eluent: petroleum ether/ethyl acetate/dichloromethane 20:1:5; $2196.4 \mathrm{mg}$, 61\%; ${ }^{1}$ H NMR $\left(400 \mathrm{MHz}, \mathrm{DMSO}-d_{6}\right) \delta 10.42(\mathrm{~s}, 1 \mathrm{H}), 7.84(\mathrm{~s}, 1 \mathrm{H}), 7.71(\mathrm{~d}, J=3.3$ $\mathrm{Hz}, 1 \mathrm{H}), 7.68-7.63(\mathrm{~m}, 3 \mathrm{H}), 7.62-7.57(\mathrm{~m}, 1 \mathrm{H}), 7.53-7.48(\mathrm{~m}, 2 \mathrm{H}), 7.38-7.32$ (m, 2H), $7.21-7.16(\mathrm{~m}, 1 \mathrm{H}), 6.99(\mathrm{td}, J=7.6,1.3 \mathrm{~Hz}, 1 \mathrm{H}), 1.84(\mathrm{~s}, 3 \mathrm{H}) ;{ }^{13} \mathbf{C}$ NMR $\left(101 \mathrm{MHz}, \mathrm{DMSO}-d_{6}\right) \delta 178.0,142.2,139.3,135.6,133.13,133.09,129.3,128.5$, 127.4, 126.7, 123.1, 120.6, 118.3, 76.9, 29.9; HRMS (ESI-TOF) Calcd for $\mathrm{C}_{17} \mathrm{H}_{17} \mathrm{~N}_{2} \mathrm{O}_{3} \mathrm{~S}_{2}[\mathrm{M}+\mathrm{H}]^{+}:$361.0675; found: 361.0682 .<smiles>Cc1ccc(S(=O)(=O)Nc2ccccc2C(C)(O)c2nccn2C)cc1</smiles>

$N$-(2-(1-hydroxy-1-(1-methyl-1 $H$-imidazol-2-yl)ethyl)phenyl)-4-methylbenzenesul fonamide (1t):

White solid; Eluent: petroleum ether/ethyl acetate/dichloromethane 20:1:10; 2635.2 mg, 71\%; ${ }^{1} \mathbf{H}$ NMR (400 MHz, DMSO-d $\left.d_{6}\right) \delta 10.49(\mathrm{~s}, 1 \mathrm{H}), 7.81-7.01(\mathrm{~m}, 8 \mathrm{H}), 6.86$ (brs, 2H), 6.42 (brs, 1H), 3.15 (s, 3H), 2.32 (s, 3H), $1.68(\mathrm{~s}, 3 \mathrm{H}) ;{ }^{13} \mathrm{C}$ NMR (101 MHz, DMSO- $\left.d_{6}\right) \delta 150.1,144.3,136.7,136.0,132.8,130.2,128.6,127.4,126.8,125.6$, 124.1, 123.9, 119.3, 75.5, 34.1, 30.4, 21.4; HRMS (ESI-TOF) Calcd for $\mathrm{C}_{19} \mathrm{H}_{22} \mathrm{~N}_{3} \mathrm{O}_{3} \mathrm{~S}$ $[\mathrm{M}+\mathrm{H}]^{+}:$372.1376; found: 372.1387 . 


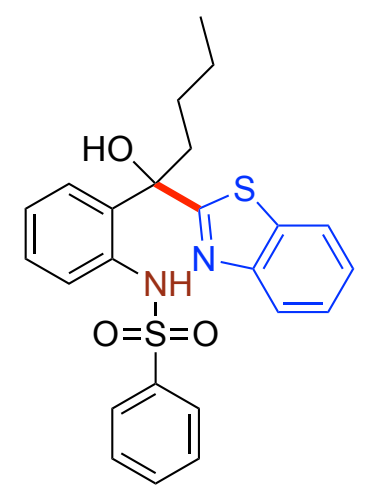

$N$-(2-(1-(benzo[d] thiazol-2-yl)-1-hydroxypentyl)phenyl)benzenesulfonamide (1u):

White solid; Eluent: petroleum ether/ethyl acetate/dichloromethane 20:1:3; $1030.8 \mathrm{mg}$, 76\%; ${ }^{1}$ H NMR $\left(400 \mathrm{MHz}, \mathrm{DMSO}-d_{6}\right) \delta 10.55(\mathrm{~s}, 1 \mathrm{H}), 8.12-8.05(\mathrm{~m}, 1 \mathrm{H}), 7.93-$ $7.86(\mathrm{~m}, 1 \mathrm{H}), 7.85(\mathrm{~s}, 1 \mathrm{H}), 7.62-7.54(\mathrm{~m}, 2 \mathrm{H}), 7.54-7.48(\mathrm{~m}, 2 \mathrm{H}), 7.49-7.39(\mathrm{~m}$, 3H), $7.26-7.16(\mathrm{~m}, 3 \mathrm{H}), 7.07-7.00(\mathrm{~m}, 1 \mathrm{H}), 2.46-2.25(\mathrm{~m}, 2 \mathrm{H}), 1.27-1.06(\mathrm{~m}$, 4H), $0.78(\mathrm{t}, J=6.9 \mathrm{~Hz}, 3 \mathrm{H}) ;{ }^{13} \mathbf{C}$ NMR $\left(101 \mathrm{MHz}, \mathrm{DMSO}-d_{6}\right) \delta 179.2,152.5,139.0$, $136.1,134.6,133.0,131.3,129.0,128.6,127.9,126.6,126.3,125.2,123.4,122.7$, 122.3, 118.4, 79.7, 40.7, 25.0, 22.2, 13.9; HRMS (ESI-TOF) Calcd for $\mathrm{C}_{24} \mathrm{H}_{25} \mathrm{~N}_{2} \mathrm{O}_{3} \mathrm{~S}_{2}$ $[\mathrm{M}+\mathrm{H}]^{+}:$453.1301; found: 453.1327 .<smiles>O=S(=O)(NNc1ccccc1C(O)(c1ccccc1)c1nc2ccccc2s1)c1ccccc1</smiles>

$N$-(2-(benzo[d] thiazol-2-yl(hydroxy)(phenyl)methyl)phenyl)benzenesulfonamide (1v): 
White solid; Eluent: petroleum ether/ethyl acetate/dichloromethane 18:1:1;1118.8 mg, 79\%; ${ }^{1}$ H NMR $\left(400 \mathrm{MHz}, \mathrm{DMSO}-d_{6}\right) \delta 9.93(\mathrm{~s}, 1 \mathrm{H}), 8.72(\mathrm{~s}, 1 \mathrm{H}), 8.18-8.08(\mathrm{~m}$, 1H), $7.96-7.88(\mathrm{~m}, 1 \mathrm{H}), 7.57-7.41(\mathrm{~m}, 4 \mathrm{H}), 7.39-7.32(\mathrm{~m}, 5 \mathrm{H}), 7.29-7.16(\mathrm{~m}$, 5H), $7.05(\mathrm{dd}, J=8.0,1.7 \mathrm{~Hz}, 1 \mathrm{H}), 6.97(\mathrm{td}, J=7.6,1.3 \mathrm{~Hz}, 1 \mathrm{H}) ;{ }^{13} \mathbf{C}$ NMR $(101$ MHz, DMSO- $\left.d_{6}\right) \delta 177.9,152.7,142.8,138.8,136.1,134.9,133.1,132.2,129.2$, $129.1,128.3,128.3,127.1,126.6,126.5,125.6,123.0,122.6,122.5,117.6,115.3$, 81.2; HRMS (ESI-TOF) Calcd for $\mathrm{C}_{26} \mathrm{H}_{21} \mathrm{~N}_{2} \mathrm{O}_{3} \mathrm{~S}_{2}[\mathrm{M}+\mathrm{H}]^{+}$: 473.0988; found: 473.0993.<smiles>O=S(=O)(Nc1ccccc1C(O)(c1ccc(F)cc1)c1nc2ccccc2s1)c1ccccc1</smiles>

$N$-(2-(benzo[d]thiazol-2-yl(4-fluorophenyl)(hydroxy)methyl)phenyl)benzenesulfo namide (1w):

White solid; Eluent: petroleum ether/ethyl acetate/dichloromethane 18:1:1; $1088.0 \mathrm{mg}$, 74\%; ${ }^{1}$ H NMR (400 MHz, DMSO-d $) \delta 9.96(\mathrm{~s}, 1 \mathrm{H}), 8.77(\mathrm{~s}, 1 \mathrm{H}), 8.17$ - $8.08(\mathrm{~m}$, 1H), $7.98-7.90(\mathrm{~m}, 1 \mathrm{H}), 7.57-7.51(\mathrm{~m}, 1 \mathrm{H}), 7.51-7.47(\mathrm{~m}, 2 \mathrm{H}), 7.47-7.41(\mathrm{~m}$, $3 \mathrm{H}), 7.32-7.14(\mathrm{~m}, 7 \mathrm{H}), 7.10(\mathrm{dd}, J=8.0,1.6 \mathrm{~Hz}, 1 \mathrm{H}), 7.00(\mathrm{td}, J=7.6,1.3 \mathrm{~Hz}, 1 \mathrm{H})$; ${ }^{13}$ C NMR (101 MHz, DMSO- $\left.d_{6}\right) \delta 177.8,163.0,160.6,152.6,139.0(\mathrm{~d}, J=3.2 \mathrm{~Hz})$, 139.0, 136.0, 134.8, 133.0, 132.3, 129.3 (d, $J=6.1 \mathrm{~Hz}), 129.1(\mathrm{~d}, J=10.8 \mathrm{~Hz}), 129.0$, $126.4,125.5,123.0,122.8,122.4,118.0,115.2,114.9,80.7 ;{ }^{19}$ F NMR (376 MHz, 
DMSO- $d_{6}$ ) $\delta$ - 114.0 ; HRMS (ESI-TOF) Calcd for $\mathrm{C}_{26} \mathrm{H}_{20} \mathrm{~N}_{2} \mathrm{O}_{3} \mathrm{~S}_{2} \mathrm{~F}[\mathrm{M}+\mathrm{H}]^{+}: 491.0894$; found: 491.0900 .

\subsection{Characterization Data of Catalysts}<smiles>c1ccc(N2c3ccccc3Sc3ccccc32)cc1</smiles>

\section{0-phenyl-10H-phenothiazine (Cat. 1):}

White solid; Eluent: petroleum ether; $594.2 \mathrm{mg}, 72 \%$; ${ }^{1} \mathbf{H}$ NMR (400 MHz, DMSO- $d_{6}$ ) $\delta 7.71-7.62(\mathrm{~m}, 2 \mathrm{H}), 7.57-7.51(\mathrm{~m}, 1 \mathrm{H}), 7.44-7.38(\mathrm{~m}, 2 \mathrm{H}), 7.08(\mathrm{dd}, J=7.5,1.7$ $\mathrm{Hz}, 2 \mathrm{H}), 6.93(\mathrm{td}, J=7.8,1.7 \mathrm{~Hz}, 2 \mathrm{H}), 6.85(\mathrm{td}, J=7.4,1.3 \mathrm{~Hz}, 2 \mathrm{H}), 6.16(\mathrm{dd}, J=8.2$, $1.3 \mathrm{~Hz}, 2 \mathrm{H}) ;{ }^{13} \mathrm{C}$ NMR (101 MHz, DMSO- $\left.d_{6}\right) \delta$ 143.6, 140.3, 131.0, 130.3, 128.4, 127.3, 126.7, 122.7, 119.4, 116.1; HRMS (ESI-TOF) Calcd for $\mathrm{C}_{18} \mathrm{H}_{14} \mathrm{NS}[\mathrm{M}+\mathrm{H}]^{+}$: 276.0841; found: 276.0812 .<smiles>Cc1ccc(N2c3ccccc3Sc3ccccc32)cc1</smiles>

10-(p-tolyl)-10H-phenothiazine (Cat. 2):

White solid; Eluent: petroleum ether; $641.8 \mathrm{mg}, 74 \%$; ${ }^{1} \mathbf{H}$ NMR (400 MHz, DMSO- $d_{6}$ ) $\delta 7.49-7.43(\mathrm{~m}, 2 \mathrm{H}), 7.32-7.26(\mathrm{~m}, 2 \mathrm{H}), 7.04(\mathrm{dd}, J=7.5,1.7 \mathrm{~Hz}, 2 \mathrm{H}), 6.90$ (ddd, 
$J=8.2,7.3,1.7 \mathrm{~Hz}, 2 \mathrm{H}), 6.83(\mathrm{td}, J=7.4,1.3 \mathrm{~Hz}, 2 \mathrm{H}), 6.14(\mathrm{dd}, J=8.2,1.3 \mathrm{~Hz}, 2 \mathrm{H})$, $2.42(\mathrm{~s}, 3 \mathrm{H}) ;{ }^{13} \mathrm{C}$ NMR $\left(101 \mathrm{MHz}, \mathrm{DMSO}-d_{6}\right) \delta 143.8,138.1,137.5,131.6,130.4$, 127.2, 126.5, 122.5, 118.9, 115.7, 20.8; HRMS (ESI-TOF) Calcd for $\mathrm{C}_{19} \mathrm{H}_{16} \mathrm{NS}$ $[\mathrm{M}+\mathrm{H}]^{+}:$290.0998; found: 290.0963 .<smiles>Brc1ccc(N2c3ccccc3Sc3ccccc32)cc1</smiles>

10-(4-bromophenyl)-10H-phenothiazine (Cat. 3):

White solid; Eluent: petroleum ether; $698.9 \mathrm{mg}, 66 \%$; ${ }^{1}$ H NMR $\left(400 \mathrm{MHz}\right.$, DMSO- $\left.d_{6}\right)$ $\delta 7.84-7.79(\mathrm{~m}, 2 \mathrm{H}), 7.39-7.33(\mathrm{~m}, 2 \mathrm{H}), 7.12(\mathrm{dd}, J=7.5,1.7 \mathrm{~Hz}, 2 \mathrm{H}), 6.98(\operatorname{td}, J$ $=7.9,1.7 \mathrm{~Hz}, 2 \mathrm{H}), 6.91(\mathrm{td}, J=7.5,1.3 \mathrm{~Hz}, 2 \mathrm{H}), 6.27(\mathrm{dd}, J=8.2,1.3 \mathrm{~Hz}, 2 \mathrm{H}) ;{ }^{13} \mathbf{C}$ NMR $\left(101 \mathrm{MHz}, \mathrm{DMSO}-d_{6}\right) \delta 143.1,140.1,133.9,131.6,127.4,126.9,123.2,120.8$, 120.6, 116.9; HRMS (ESI-TOF) Calcd for $\mathrm{C}_{18} \mathrm{H}_{13} \mathrm{NSBr}[\mathrm{M}+\mathrm{H}]^{+}$: 353.9947; found: 353.9896.<smiles>CC(C)(C)c1ccc(N2c3ccccc3Sc3ccccc32)cc1</smiles>

10-(4-(tert-butyl)phenyl)-10H-phenothiazine (Cat. 4): 
White solid; Eluent: petroleum ether; $615.9 \mathrm{mg}, 62 \% ;{ }^{1} \mathbf{H}$ NMR (400 MHz, DMSO- $d_{6}$ ) $\delta 7.71-7.63(\mathrm{~m}, 2 \mathrm{H}), 7.37-7.30(\mathrm{~m}, 2 \mathrm{H}), 7.06(\mathrm{dd}, J=7.5,1.6 \mathrm{~Hz}, 2 \mathrm{H}), 6.92(\mathrm{td}, J$ $=7.9,1.7 \mathrm{~Hz}, 2 \mathrm{H}), 6.84(\mathrm{td}, J=7.4,1.3 \mathrm{~Hz}, 2 \mathrm{H}), 6.14(\mathrm{dd}, J=8.2,1.3 \mathrm{~Hz}, 2 \mathrm{H}), 1.37$ $(\mathrm{s}, 9 \mathrm{H}) ;{ }^{13} \mathrm{C}$ NMR $\left(101 \mathrm{MHz}, \mathrm{DMSO}-d_{6}\right) \delta 150.9,143.8,137.6,129.9,127.8,127.3$, 126.6, 122.6, 119.1, 115.8, 34.5, 31.1; HRMS (ESI-TOF) Calcd for $\mathrm{C}_{22} \mathrm{H}_{22} \mathrm{NS}$ $[\mathrm{M}+\mathrm{H}]^{+}:$332.1467; found: 332.1444.

\subsection{Characterization Data of Products 2}

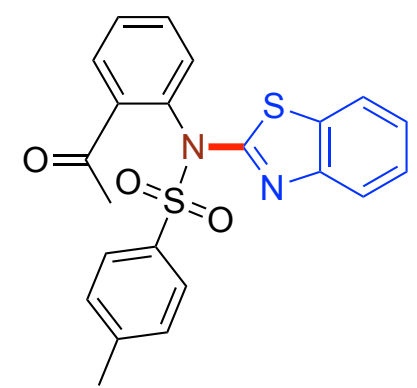

$N$-(2-acetylphenyl)- $N$-(benzo $[d]$ thiazol-2-yl)-4-methylbenzenesulfonamide (2a):

White solid; Eluent: petroleum ether/ethyl acetate 18:1; $117.7 \mathrm{mg}, 93 \% ;{ }^{1} \mathbf{H}$ NMR (400 MHz, DMSO- $\left.d_{6}\right) \delta 7.96(\mathrm{dd}, J=17.6,7.6 \mathrm{~Hz}, 2 \mathrm{H}), 7.81(\mathrm{~d}, J=7.9 \mathrm{~Hz}, 2 \mathrm{H})$, $7.75-7.57(\mathrm{~m}, 3 \mathrm{H}), 7.54-7.24(\mathrm{~m}, 4 \mathrm{H}), 7.12(\mathrm{~d}, J=7.5 \mathrm{~Hz}, 1 \mathrm{H}), 2.53(\mathrm{~s}, 3 \mathrm{H}), 2.41$ $(\mathrm{s}, 3 \mathrm{H}) ;{ }^{13} \mathbf{C}$ NMR $\left(101 \mathrm{MHz}, \mathrm{DMSO}-d_{6}\right) \delta 198.6,161.5,149.6,145.6,139.2,134.6$, $134.4,132.7,132.4,131.5,130.5,120.99,129.96,128.4,126.4,124.3,121.6,121.3$, 29.5, 21.2; HRMS (ESI-TOF) Calcd for $\mathrm{C}_{22} \mathrm{H}_{19} \mathrm{~N}_{2} \mathrm{O}_{3} \mathrm{~S}_{2}[\mathrm{M}+\mathrm{H}]^{+}$: 423.0832; found: 423.0859 . 


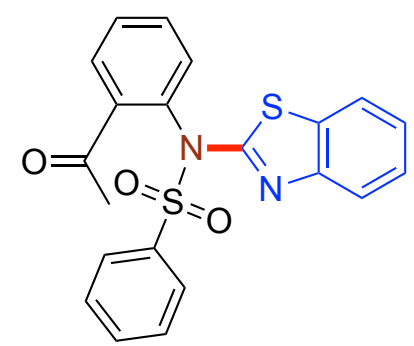

$N$-(2-acetylphenyl)- $N$-(benzo[d] thiazol-2-yl)benzenesulfonamide (2b):

Yellow solid; Eluent: petroleum ether/ethyl acetate 18:1; $110.2 \mathrm{mg}, 90 \%$; ${ }^{1} \mathbf{H}$ NMR $\left(400 \mathrm{MHz}, \mathrm{DMSO}-d_{6}\right) \delta 8.00(\mathrm{dd}, J=7.6,1.7 \mathrm{~Hz}, 1 \mathrm{H}), 7.96-7.90(\mathrm{~m}, 3 \mathrm{H}), 7.82-$ $7.77(\mathrm{~m}, 1 \mathrm{H}), 7.72-7.62(\mathrm{~m}, 5 \mathrm{H}), 7.43-7.37(\mathrm{~m}, 1 \mathrm{H}), 7.34-7.28(\mathrm{~m}, 1 \mathrm{H}), 7.12(\mathrm{dd}$, $J=7.8,1.4 \mathrm{~Hz}, 1 \mathrm{H}), 2.52(\mathrm{~s}, 3 \mathrm{H}) ;{ }^{13} \mathbf{C}$ NMR $\left(101 \mathrm{MHz}, \mathrm{DMSO}-d_{6}\right) \delta$ 198.5, 161.5, $149.5,139.1,137.3,134.8,134.5,132.7,132.4,131.5,130.6,130.1,129.6,128.3$, 126.4, 124.3, 121.7, 121.4, 29.4; HRMS (ESI-TOF) Calcd for $\mathrm{C}_{21} \mathrm{H}_{17} \mathrm{~N}_{2} \mathrm{O}_{3} \mathrm{~S}_{2}[\mathrm{M}+\mathrm{H}]^{+}$: 409.0675; found: 409.0694 .

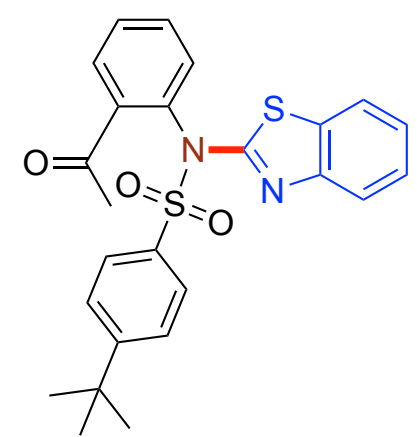

$\mathrm{N}$-(2-acetylphenyl)- $\mathrm{N}$-(benzo[d]thiazol-2-yl)-4-(tert-butyl)benzenesulfonamide (2c):

White solid; Eluent: petroleum ether/ethyl acetate 18:1; $126.7 \mathrm{mg}, 91 \% ;{ }^{1} \mathbf{H}$ NMR $\left(400 \mathrm{MHz}, \mathrm{DMSO}-d_{6}\right) \delta 8.02-7.96(\mathrm{~m}, 1 \mathrm{H}), 7.93(\mathrm{dd}, J=8.0,0.8 \mathrm{~Hz}, 1 \mathrm{H}), 7.88-$ $7.82(\mathrm{~m}, 2 \mathrm{H}), 7.73-7.63(\mathrm{~m}, 5 \mathrm{H}), 7.43-7.36(\mathrm{~m}, 1 \mathrm{H}), 7.33-7.27(\mathrm{~m}, 1 \mathrm{H}), 7.18-$ 
$7.12(\mathrm{~m}, 1 \mathrm{H}), 2.49(\mathrm{~s}, 3 \mathrm{H}), 1.28(\mathrm{~s}, 9 \mathrm{H}) ;{ }^{13} \mathrm{C}$ NMR (101 MHz, DMSO-d 6 ) $\delta$ 198.4, $161.5,158.0,149.6,139.0,134.6,134.5,132.7,132.4,131.5,130.5,130.0,128.2$ 126.44, 126.37, 124.2, 121.6, 121.3, 35.1, 30.7, 29.4; HRMS (ESI-TOF) Calcd for $\mathrm{C}_{25} \mathrm{H}_{25} \mathrm{~N}_{2} \mathrm{O}_{3} \mathrm{~S}_{2}[\mathrm{M}+\mathrm{H}]^{+}:$465.1301; found: 465.1321.

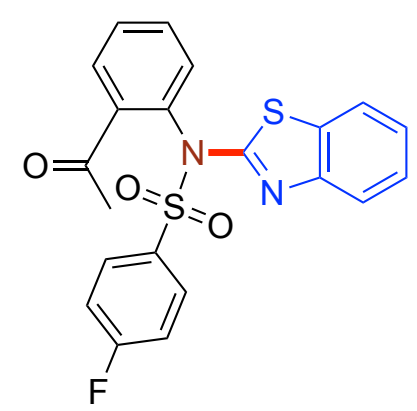

$N$-(2-acetylphenyl)- $N$-(benzo[d] thiazol-2-yl)-4-fluorobenzenesulfonamide (2d):

Light brown oil; Eluent: petroleum ether/ethyl acetate $18: 1 ; 112.5 \mathrm{mg}, 88 \% ;{ }^{1} \mathbf{H}$ NMR $\left(400 \mathrm{MHz}, \mathrm{DMSO}-d_{6}\right) \delta 8.07-7.97(\mathrm{~m}, 3 \mathrm{H}), 7.92(\mathrm{ddd}, J=8.0,1.3,0.6 \mathrm{~Hz}, 1 \mathrm{H})$, $7.75-7.64(\mathrm{~m}, 3 \mathrm{H}), 7.54-7.47(\mathrm{~m}, 2 \mathrm{H}), 7.45-7.39(\mathrm{~m}, 1 \mathrm{H}), 7.34-7.28(\mathrm{~m}, 1 \mathrm{H})$, $7.23-7.18(\mathrm{~m}, 1 \mathrm{H}), 2.53(\mathrm{~s}, 3 \mathrm{H}) ;{ }^{13} \mathrm{C}$ NMR (101 MHz, DMSO-d $)$ ) 198.5, 166.7, 164.2, 161.5, 149.6, 138.9, 134.4, 133.6 (d, $J=2.9 \mathrm{~Hz}), 132.9,132.4,131.9,131.8$, $131.7,130.7,130.2,126.5,124.4,121.7,121.4,116.9,116.7,29.4 ;{ }^{19}$ F NMR $(376$ MHz, DMSO- $d_{6}$ ) $\delta-103.1$; HRMS (ESI-TOF) Calcd for $\mathrm{C}_{21} \mathrm{H}_{16} \mathrm{~N}_{2} \mathrm{O}_{3} \mathrm{~S}_{2} \mathrm{~F}[\mathrm{M}+\mathrm{H}]^{+}$: 427.0581; found: 427.0604 . 


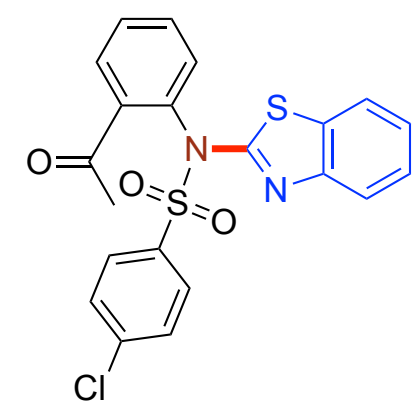

$N$-(2-acetylphenyl)- $N$-(benzo[ $[d]$ thiazol-2-yl)-4-chlorobenzenesulfonamide (2e):

Yellow oil; Eluent: petroleum ether/ethyl acetate 18:1; $120.7 \mathrm{mg}, 91 \%$; ${ }^{1}$ H NMR (400 MHz, DMSO- $\left.d_{6}\right) \delta 8.02(\mathrm{dd}, J=7.3,2.0 \mathrm{~Hz}, 1 \mathrm{H}), 7.99-7.90(\mathrm{~m}, 3 \mathrm{H}), 7.79-7.65(\mathrm{~m}$, 5H), $7.46-7.39(\mathrm{~m}, 1 \mathrm{H}), 7.31(\mathrm{td}, J=7.7,1.2 \mathrm{~Hz}, 1 \mathrm{H}), 7.23(\mathrm{dd}, J=7.5,1.6 \mathrm{~Hz}, 1 \mathrm{H})$, $2.53(\mathrm{~s}, 3 \mathrm{H}) ;{ }^{13} \mathrm{C}$ NMR $\left(101 \mathrm{MHz}, \mathrm{DMSO}-d_{6}\right) \delta 198.6,161.5,149.7,139.8,138.9$, $136.2,134.3,133.0,132.5,131.7,130.8,130.5,130.2,129.7,126.5,124.4,121.7$ 121.4, 29.4; HRMS (ESI-TOF) Calcd for $\mathrm{C}_{21} \mathrm{H}_{16} \mathrm{~N}_{2} \mathrm{O}_{3} \mathrm{~S}_{2} \mathrm{Cl}[\mathrm{M}+\mathrm{H}]^{+}$: 443.0258; found: 443.0272.

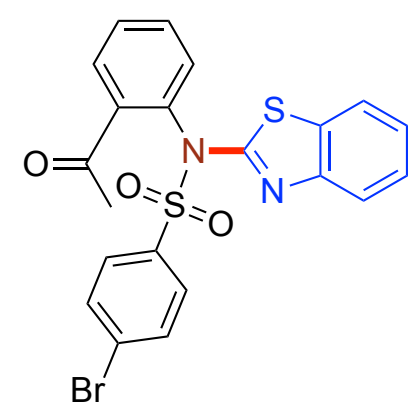

$N$-(2-acetylphenyl)- $N$-(benzo $[d]$ thiazol-2-yl)-4-bromobenzenesulfonamide (2f):

White solid; Eluent: petroleum ether/ethyl acetate $18: 1 ; 126.8 \mathrm{mg}, 87 \% ;{ }^{1} \mathbf{H}$ NMR $\left(400 \mathrm{MHz}, \mathrm{DMSO}-d_{6}\right) \delta 8.05-8.00(\mathrm{~m}, 1 \mathrm{H}), 7.95-7.85(\mathrm{~m}, 5 \mathrm{H}), 7.75-7.65(\mathrm{~m}$, 3H), $7.45-7.39(\mathrm{~m}, 1 \mathrm{H}), 7.34-7.29(\mathrm{~m}, 1 \mathrm{H}), 7.26-7.22(\mathrm{~m}, 1 \mathrm{H}), 2.53(\mathrm{~s}, 3 \mathrm{H}) ;{ }^{13} \mathrm{C}$ NMR $\left(101 \mathrm{MHz}, \mathrm{DMSO}-d_{6}\right) \delta 198.5,161.5,149.6,138.8,136.6,134.3,133.0,132.6$, 
$132.4,131.6,130.7,130.4,130.2,128.9,126.5,124.4,121.7,121.4,29.4$; HRMS (ESI-TOF) Calcd for $\mathrm{C}_{21} \mathrm{H}_{16} \mathrm{~N}_{2} \mathrm{O}_{3} \mathrm{~S}_{2} \mathrm{Br}[\mathrm{M}+\mathrm{H}]^{+}$: 486.9780; found: 486.9777.

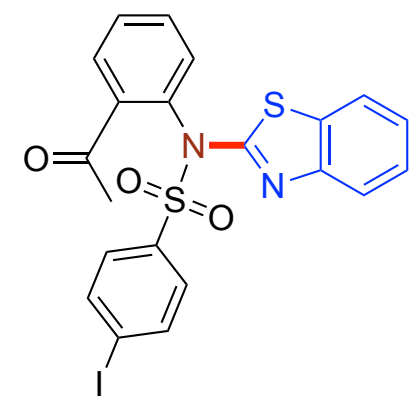

$N$-(2-acetylphenyl)- $N$-(benzo[ $d]$ thiazol-2-yl)-4-iodobenzenesulfonamide (2g):

White solid; Eluent: petroleum ether/ethyl acetate $18: 1 ; 137.7 \mathrm{mg}, 86 \%$; ${ }^{1} \mathbf{H}$ NMR $\left(400 \mathrm{MHz}, \mathrm{DMSO}-d_{6}\right) \delta 8.10-8.03(\mathrm{~m}, 2 \mathrm{H}), 8.01(\mathrm{dd}, J=7.2,2.0 \mathrm{~Hz}, 1 \mathrm{H}), 7.95-$ $7.90(\mathrm{~m}, 1 \mathrm{H}), 7.77-7.63(\mathrm{~m}, 5 \mathrm{H}), 7.45-7.39(\mathrm{~m}, 1 \mathrm{H}), 7.34-7.28(\mathrm{~m}, 1 \mathrm{H}), 7.25-$ $7.19(\mathrm{~m}, 1 \mathrm{H}), 2.53(\mathrm{~s}, 3 \mathrm{H}) ;{ }^{13} \mathrm{C}$ NMR $\left(101 \mathrm{MHz}, \mathrm{DMSO}-d_{6}\right) \delta 198.5,161.5,149.6$ $138.9,138.4,137.0,134.3,133.0,132.4,131.6,130.7,130.2,129.9,126.5,124.4$, 121.7, 121.4, 103.8, 29.4; HRMS (ESI-TOF) Calcd for $\mathrm{C}_{21} \mathrm{H}_{16} \mathrm{~N}_{2} \mathrm{O}_{3} \mathrm{~S}_{2} \mathrm{I}[\mathrm{M}+\mathrm{H}]^{+}$: 534.9642; found: 534.9635 .

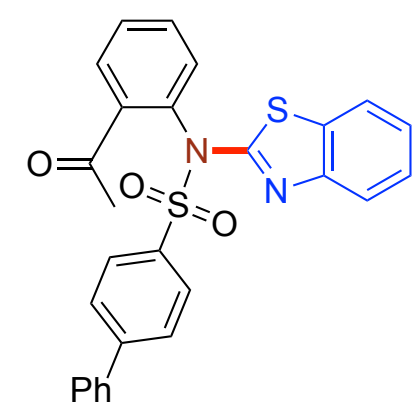

$N$-(2-acetylphenyl)- $N$-(benzo[d] thiazol-2-yl)-[1,1'-biphenyl]-4-sulfonamide (2h): 
Yellow oil; Eluent: petroleum ether/ethyl acetate 18:1; $120.5 \mathrm{mg}, 83 \% ;{ }^{1} \mathbf{H}$ NMR (400 MHz, DMSO- $\left.d_{6}\right) \delta 8.03-7.92(\mathrm{~m}, 6 \mathrm{H}), 7.78-7.65(\mathrm{~m}, 5 \mathrm{H}), 7.54-7.38(\mathrm{~m}, 4 \mathrm{H})$, $7.34-7.28(\mathrm{~m}, 1 \mathrm{H}), 7.23-7.19(\mathrm{~m}, 1 \mathrm{H}), 2.53(\mathrm{~s}, 3 \mathrm{H}) ;{ }^{13} \mathbf{C}$ NMR (101 MHz, DMSO- $\left.d_{6}\right) \delta 198.5,161.5,149.6,146.0,139.0,137.9,136.0,134.5,132.8,132.4$ $131.6,130.6,130.1,129.2,129.0,128.9,127.6,127.2,126.4,124.3,121.6,121.3$, 29.4; HRMS (ESI-TOF) Calcd for $\mathrm{C}_{27} \mathrm{H}_{21} \mathrm{~N}_{2} \mathrm{O}_{3} \mathrm{~S}_{2}[\mathrm{M}+\mathrm{H}]^{+}$: 485.0988; found: 485.1012 .

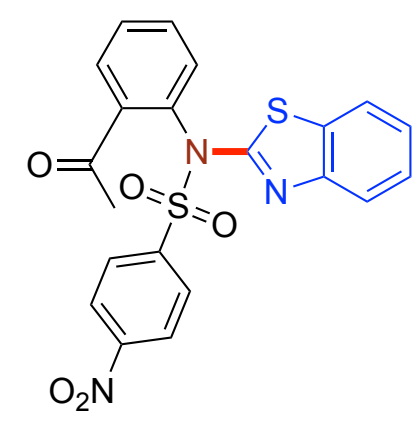

$N$-(2-acetylphenyl)- $N$-(benzo[d] thiazol-2-yl)-4-nitrobenzenesulfonamide (2i):

Light brown oil; Eluent: petroleum ether/ethyl acetate 16:1; $104.6 \mathrm{mg}, 77 \%$; ${ }^{1} \mathbf{H}$ NMR $\left(400 \mathrm{MHz}, \mathrm{DMSO}-d_{6}\right) \delta 8.49-8.44(\mathrm{~m}, 2 \mathrm{H}), 8.27-8.22(\mathrm{~m}, 2 \mathrm{H}), 8.07(\mathrm{dd}, J=7.6$, $1.8 \mathrm{~Hz}, 1 \mathrm{H}), 7.92(\mathrm{dd}, J=8.1,1.2 \mathrm{~Hz}, 1 \mathrm{H}), 7.79-7.76(\mathrm{~m}, 1 \mathrm{H}), 7.75-7.67(\mathrm{~m}, 2 \mathrm{H})$, $7.47-7.41(\mathrm{~m}, 1 \mathrm{H}), 7.35-7.26(\mathrm{~m}, 2 \mathrm{H}), 2.55(\mathrm{~s}, 3 \mathrm{H}) ;{ }^{13} \mathbf{C}$ NMR (101 MHz, DMSO- $\left.d_{6}\right) \delta 198.6,161.4,150.7,149.7,142.7,138.7,134.1,133.2,132.5,131.8$ 131.0, 130.5, 130.4, 126.6, 124.7, 124.6, 121.8, 121.6, 29.4; HRMS (ESI-TOF) Calcd for $\mathrm{C}_{21} \mathrm{H}_{16} \mathrm{~N}_{3} \mathrm{O}_{5} \mathrm{~S}_{2}[\mathrm{M}+\mathrm{H}]^{+}:$454.0526; found: 454.0543 . 


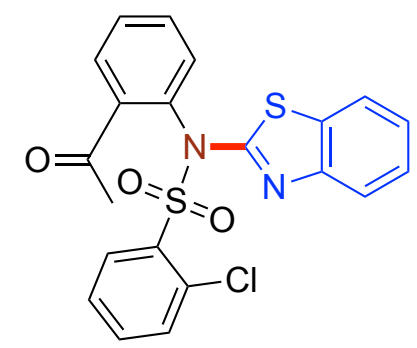

$N$-(2-acetylphenyl)- $N$-(benzo[d] thiazol-2-yl)-2-chlorobenzenesulfonamide (2j):

Yellow solid; Eluent: petroleum ether/ethyl acetate 18:1; $116.7 \mathrm{mg}, 88 \%$; ${ }^{1} \mathbf{H}$ NMR $\left(400 \mathrm{MHz}, \mathrm{DMSO}-d_{6}\right) \delta 8.01-7.93(\mathrm{~m}, 3 \mathrm{H}), 7.77-7.71(\mathrm{~m}, 2 \mathrm{H}), 7.66-7.59(\mathrm{~m}$, 2H), $7.58-7.52(\mathrm{~m}, 2 \mathrm{H}), 7.37(\mathrm{td}, J=7.3,1.4 \mathrm{~Hz}, 1 \mathrm{H}), 7.31(\mathrm{td}, J=7.6,1.3 \mathrm{~Hz}, 1 \mathrm{H})$, $7.17(\mathrm{dd}, J=7.9,1.3 \mathrm{~Hz}, 1 \mathrm{H}), 2.51(\mathrm{~s}, 3 \mathrm{H}) ;{ }^{13} \mathbf{C}$ NMR (101 MHz, DMSO-d 6 ) $\delta$ 198.5, $162.2,148.9,138.8,136.0,135.1,133.5,132.9,132.7,132.5,132.4,132.2,131.5$, 130.5, 130.0, 128.0, 126.2, 124.3, 121.6, 121.3, 29.4; HRMS (ESI-TOF) Calcd for $\mathrm{C}_{21} \mathrm{H}_{16} \mathrm{~N}_{2} \mathrm{O}_{3} \mathrm{~S}_{2} \mathrm{Cl}[\mathrm{M}+\mathrm{H}]^{+}$: 443.0285; found: 443.0311.

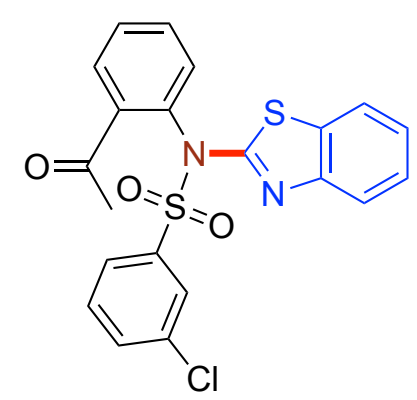

$N$-(2-acetylphenyl)- $N$-(benzo[ $[d]$ thiazol-2-yl)-3-chlorobenzenesulfonamide (2k):

Yellow solid; Eluent: petroleum ether/ethyl acetate $18: 1 ; 114.0 \mathrm{mg}, 86 \%$; ${ }^{\mathbf{1}} \mathbf{H}$ NMR $\left(400 \mathrm{MHz}, \mathrm{DMSO}-d_{6}\right) \delta 8.07-8.02(\mathrm{~m}, 1 \mathrm{H}), 7.99(\mathrm{t}, J=2.0 \mathrm{~Hz}, 1 \mathrm{H}), 7.94-7.86(\mathrm{~m}$, 3H), $7.75-7.66(\mathrm{~m}, 4 \mathrm{H}), 7.46-7.40(\mathrm{~m}, 1 \mathrm{H}), 7.34-7.26(\mathrm{~m}, 2 \mathrm{H}), 2.53(\mathrm{~s}, 3 \mathrm{H}) ;{ }^{13} \mathrm{C}$ NMR (101 MHz, DMSO-d $\left.d_{6}\right) \delta 198.5,161.5,149.7,139.1,138.7,134.6,134.2,133.8$, $133.0,132.4,131.8,131.5,130.9,130.3,128.2,127.1,126.5,124.4,121.7,121.4$ 
29.4; HRMS (ESI-TOF) Calcd for $\mathrm{C}_{21} \mathrm{H}_{16} \mathrm{~N}_{2} \mathrm{O}_{3} \mathrm{~S}_{2} \mathrm{Cl}[\mathrm{M}+\mathrm{H}]^{+}$: 443.0285; found: 443.0311.

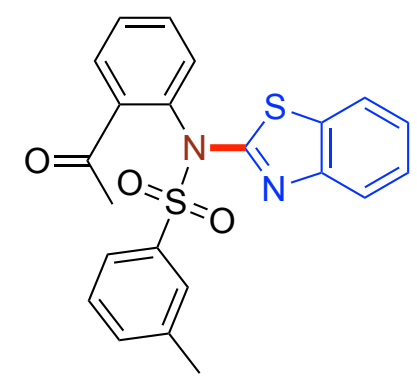

$N$-(2-acetylphenyl)- $N$-(benzo $[d]$ thiazol-2-yl)-3-methylbenzenesulfonamide (2l):

White solid; Eluent: petroleum ether/ethyl acetate 18:1; $115.2 \mathrm{mg}, 91 \%$; ${ }^{1} \mathbf{H}$ NMR $\left(400 \mathrm{MHz}, \mathrm{DMSO}-d_{6}\right) \delta 8.01-7.96(\mathrm{~m}, 1 \mathrm{H}), 7.96-7.91(\mathrm{~m}, 1 \mathrm{H}), 7.75-7.63(\mathrm{~m}$, 5H), $7.62-7.57(\mathrm{~m}, 1 \mathrm{H}), 7.53(\mathrm{t}, J=7.6 \mathrm{~Hz}, 1 \mathrm{H}), 7.43-7.37(\mathrm{~m}, 1 \mathrm{H}), 7.34-7.27(\mathrm{~m}$, 1H), $7.16-7.12(\mathrm{~m}, 1 \mathrm{H}), 2.52(\mathrm{~s}, 3 \mathrm{H}), 2.39(\mathrm{~s}, 3 \mathrm{H}) ;{ }^{13} \mathbf{C}$ NMR (101 MHz, DMSO-d $)$ $\delta 198.4,161.5,149.5,139.3,139.1,137.2,135.3,134.5,132.6,132.4,131.6,130.5$, 130.0, 129.3, 128.4, 126.4, 125.4, 124.2, 121.6, 121.3, 29.4, 20.8; HRMS (ESI-TOF) Calcd for $\mathrm{C}_{22} \mathrm{H}_{19} \mathrm{~N}_{2} \mathrm{O}_{3} \mathrm{~S}_{2}[\mathrm{M}+\mathrm{H}]^{+}$: 423.0832; found: 423.0828 .<smiles>CC(=O)c1ccccc1N(c1nc2ccccc2s1)S(=O)(=O)c1cccnc1</smiles>

$N$-(2-acetylphenyl)- $N$-(benzo[d] thiazol-2-yl)pyridine-3-sulfonamide (2m):

White solid; Eluent: petroleum ether/ethyl acetate $16: 1 ; 100.6 \mathrm{mg}, 82 \%$; ${ }^{1} \mathbf{H}$ NMR $\left(400 \mathrm{MHz}, \mathrm{DMSO}-d_{6}\right) \delta 9.12(\mathrm{dd}, J=2.5,0.8 \mathrm{~Hz}, 1 \mathrm{H}), 8.93(\mathrm{dd}, J=4.8,1.6 \mathrm{~Hz}, 1 \mathrm{H})$, 
$8.41(\mathrm{ddd}, J=8.2,2.4,1.6 \mathrm{~Hz}, 1 \mathrm{H}), 8.10-8.03(\mathrm{~m}, 1 \mathrm{H}), 7.90(\mathrm{dd}, J=8.1,1.2 \mathrm{~Hz}$, 1H), $7.79-7.68(\mathrm{~m}, 4 \mathrm{H}), 7.45-7.39(\mathrm{~m}, 1 \mathrm{H}), 7.38-7.34(\mathrm{~m}, 1 \mathrm{H}), 7.33-7.27(\mathrm{~m}$, 1H), $2.55(\mathrm{~s}, 3 \mathrm{H}) ;{ }^{13} \mathrm{C}$ NMR (101 MHz, DMSO- $\left.d_{6}\right) \delta 198.5,161.5,154.8,149.8$, $148.8,138.6,136.9,134.3,134.1,133.2,132.4,131.8,131.0,130.4,126.5,124.4$, 124.3, 121.7, 121.4, 29.3; HRMS (ESI-TOF) Calcd for $\mathrm{C}_{20} \mathrm{H}_{16} \mathrm{~N}_{3} \mathrm{O}_{3} \mathrm{~S}_{2}[\mathrm{M}+\mathrm{H}]^{+}$: 410.0628; found: 410.0626 .<smiles>CC(=O)c1ccccc1N(c1nc2ccccc2s1)S(=O)(=O)c1cccs1</smiles>

$N$-(2-acetylphenyl)- $N$-(benzo[d] thiazol-2-yl)thiophene-2-sulfonamide (2n):

Yellow oil; Eluent: petroleum ether/ethyl acetate $18: 1 ; 106.8 \mathrm{mg}, 86 \% ;{ }^{1} \mathbf{H}$ NMR (400 MHz, DMSO- $\left.d_{6}\right) \delta 8.16(\mathrm{dd}, J=5.0,1.4 \mathrm{~Hz}, 1 \mathrm{H}), 8.03-7.98(\mathrm{~m}, 1 \mathrm{H}), 7.96(\mathrm{ddd}, J=$ 8.0, 1.3, 0.6 Hz, 1H), $7.88(\mathrm{dd}, J=3.9,1.4 \mathrm{~Hz}, 1 \mathrm{H}), 7.76-7.71(\mathrm{~m}, 1 \mathrm{H}), 7.71-7.65$ (m, 2H), $7.44-7.39(\mathrm{~m}, 1 \mathrm{H}), 7.35-7.29(\mathrm{~m}, 1 \mathrm{H}), 7.27$ (dd, $J=5.0,3.9 \mathrm{~Hz}, 1 \mathrm{H}), 7.22$ $-7.18(\mathrm{~m}, 1 \mathrm{H}), 2.53(\mathrm{~s}, 3 \mathrm{H}) ;{ }^{13} \mathbf{C}$ NMR (101 MHz, DMSO-d $) \delta 198.5,161.2,149.6$, $139.0,136.6,136.5,135.9,134.5,132.8,132.5,131.2,130.6,130.1,128.1,126.5$, 124.4, 121.7, 121.4, 29.4; HRMS (ESI-TOF) Calcd for $\mathrm{C}_{19} \mathrm{H}_{15} \mathrm{~N}_{2} \mathrm{O}_{3} \mathrm{~S}_{3}[\mathrm{M}+\mathrm{H}]^{+}$: 415.0239; found: 415.0263 . 


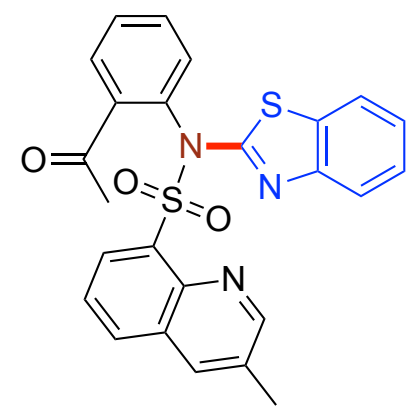

$N$-(2-acetylphenyl)- $N$-(benzo[d] thiazol-2-yl)-3-methylquinoline-8-sulfonamide (20):

Light brown solid; Eluent: petroleum ether/ethyl acetate $16: 1 ; 112.1 \mathrm{mg}, 79 \% ;{ }^{1} \mathbf{H}$ NMR $\left(400 \mathrm{MHz}, \mathrm{DMSO}-d_{6}\right) \delta 8.47(\mathrm{~d}, J=2.2 \mathrm{~Hz}, 1 \mathrm{H}), 8.33-8.28(\mathrm{~m}, 2 \mathrm{H}), 8.24(\mathrm{dd}$, $J=7.5,1.4 \mathrm{~Hz}, 1 \mathrm{H}), 8.02-7.97(\mathrm{~m}, 1 \mathrm{H}), 7.88(\mathrm{dd}, J=7.7,1.6 \mathrm{~Hz}, 1 \mathrm{H}), 7.70(\mathrm{t}, J=$ $7.8 \mathrm{~Hz}, 1 \mathrm{H}), 7.58-7.51(\mathrm{~m}, 2 \mathrm{H}), 7.41-7.28(\mathrm{~m}, 3 \mathrm{H}), 6.78(\mathrm{dd}, J=8.0,1.2 \mathrm{~Hz}, 1 \mathrm{H})$, $2.51(\mathrm{~s}, 3 \mathrm{H}), 2.43$ (s, 3H); ${ }^{13} \mathrm{C}$ NMR (101 MHz, DMSO-d $\left.{ }_{6}\right) \delta$ 198.6, 163.5, 153.1, $148.7,141.1,139.5,135.4,135.0,134.3,134.1,133.1,132.8,132.6,131.81,131.78$, 129.9, 129.4, 128.5, 125.7, 123.8, 121.3, 120.9, 29.5, 18.0; HRMS (ESI-TOF) Calcd for $\mathrm{C}_{25} \mathrm{H}_{20} \mathrm{~N}_{3} \mathrm{O}_{3} \mathrm{~S}_{2}[\mathrm{M}+\mathrm{H}]^{+}$: 474.0941; found: 474.0952.<smiles>CCS(=O)(=O)N(c1nc2ccccc2s1)c1ccccc1C(C)=O</smiles>

\section{$N$-(2-acetylphenyl)- $N$-(benzo[d]thiazol-2-yl)ethanesulfonamide (2p):}

Yellow oil; Eluent: petroleum ether/ethyl acetate 16:1; $93.9 \mathrm{mg}, 87 \% ;{ }^{\mathbf{1}} \mathbf{H}$ NMR (400 MHz, DMSO- $\left.d_{6}\right) \delta 8.00(\mathrm{dd}, J=7.4,1.7 \mathrm{~Hz}, 1 \mathrm{H}), 7.90(\mathrm{dd}, J=8.1,1.2 \mathrm{~Hz}, 1 \mathrm{H}), 7.79$ - $7.66(\mathrm{~m}, 4 \mathrm{H}), 7.46-7.40(\mathrm{~m}, 1 \mathrm{H}), 7.33-7.27$ (m, 1H), 3.91 (q, $J=7.3 \mathrm{~Hz}, 2 \mathrm{H}), 2.53$ 
(s, 3H), 1.37 (t, $J=7.4 \mathrm{~Hz}, 3 \mathrm{H}) ;{ }^{13} \mathrm{C}$ NMR (101 MHz, DMSO-d $)_{6} \delta$ 199.0, 162.8, $150.1,138.8,134.6,133.0,132.23,132.17,130.5,130.0,126.5,124.1,121.6,121.2$ 48.5, 29.4, 7.5; HRMS (ESI-TOF) Calcd for $\mathrm{C}_{17} \mathrm{H}_{17} \mathrm{~N}_{2} \mathrm{O}_{3} \mathrm{~S}_{2}[\mathrm{M}+\mathrm{H}]^{+}:$: 361.0675; found: 361.0697.<smiles>CC(=O)c1ccccc1N(c1nc2ccccc2s1)S(=O)(=O)C1CC1</smiles>

$N$-(2-acetylphenyl)- $N$-(benzo[ $d]$ thiazol-2-yl)cyclopropanesulfonamide (2q):

White solid; Eluent: petroleum ether/ethyl acetate $16: 1 ; 94.8 \mathrm{mg}, 85 \% ;{ }^{1} \mathbf{H}$ NMR (400 MHz, DMSO- $\left.d_{6}\right) \delta 7.99-7.90(\mathrm{~m}, 2 \mathrm{H}), 7.79-7.62(\mathrm{~m}, 4 \mathrm{H}), 7.46-7.40(\mathrm{~m}, 1 \mathrm{H}), 7.34$ - $7.28(\mathrm{~m}, 1 \mathrm{H}), 3.52-3.42(\mathrm{~m}, 1 \mathrm{H}), 2.52(\mathrm{~s}, 3 \mathrm{H}), 1.29-1.03(\mathrm{~m}, 4 \mathrm{H}) ;{ }^{13} \mathbf{C}$ NMR $(101$ MHz, DMSO- $\left.d_{6}\right) \delta 198.8,162.4,149.9,138.9,135.0,132.8,132.4,132.1,130.4$, 129.9, 126.5, 124.2, 121.6, 121.2, 31.1, 29.5, 6.1; HRMS (ESI-TOF) Calcd for $\mathrm{C}_{18} \mathrm{H}_{17} \mathrm{~N}_{2} \mathrm{O}_{3} \mathrm{~S}_{2}[\mathrm{M}+\mathrm{H}]^{+}:$373.0675; found: 373.0699 .<smiles>COc1ccc2nc(N(c3ccccc3C(C)=O)S(=O)(=O)c3ccccc3)sc2c1</smiles>

$N$-(2-acetylphenyl)- $N$-(6-methoxybenzo[ $d]$ thiazol-2-yl)benzenesulfonamide (2r):

Yellow oil; Eluent: petroleum ether/ethyl acetate 18:1; $120.9 \mathrm{mg}, 92 \% ;{ }^{1} \mathbf{H}$ NMR (400 MHz, DMSO- $\left.d_{6}\right) \delta 7.96(\mathrm{dd}, J=7.5,1.8 \mathrm{~Hz}, 1 \mathrm{H}), 7.92-7.85(\mathrm{~m}, 2 \mathrm{H}), 7.81-7.74(\mathrm{~m}$, 
1H), $7.69-7.58(\mathrm{~m}, 5 \mathrm{H}), 7.55(\mathrm{~d}, J=2.6 \mathrm{~Hz}, 1 \mathrm{H}), 7.09(\mathrm{dd}, J=7.6,1.5 \mathrm{~Hz}, 1 \mathrm{H}), 7.00$ $(\mathrm{dd}, J=8.9,2.6 \mathrm{~Hz}, 1 \mathrm{H}), 3.77(\mathrm{~s}, 3 \mathrm{H}), 2.54(\mathrm{~s}, 3 \mathrm{H}) ;{ }^{13} \mathbf{C}$ NMR (101 MHz, DMSO-d $)$ $\delta 198.7,159.2,156.7,143.6,139.4,137.4,134.7,134.6,134.2,132.6,131.4,130.4$ 129.9, 129.6, 128.2, 122.2, 115.1, 104.8, 55.7, 29.5; HRMS (ESI-TOF) Calcd for $\mathrm{C}_{22} \mathrm{H}_{19} \mathrm{~N}_{2} \mathrm{O}_{4} \mathrm{~S}_{2}[\mathrm{M}+\mathrm{H}]^{+}:$439.0781; found: 439.0806 .

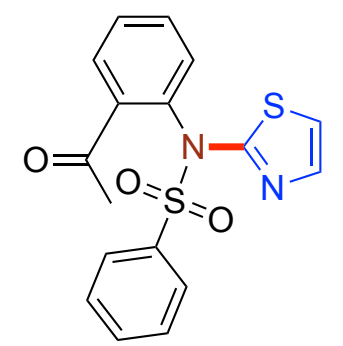

$N$-(2-acetylphenyl)- $N$-(thiazol-2-yl)benzenesulfonamide (2s):

Yellow oil; Eluent: petroleum ether/ethyl acetate $18: 1 ; 97.7 \mathrm{mg}, 91 \% ;{ }^{1} \mathbf{H}$ NMR (400 MHz, DMSO-d $\left.d_{6}\right) \delta .89(\mathrm{dd}, J=7.6,1.7 \mathrm{~Hz}, 1 \mathrm{H}), 7.80-7.71(\mathrm{~m}, 3 \mathrm{H}), 7.67-7.53(\mathrm{~m}$, 4H), $7.53-7.46(\mathrm{~m}, 2 \mathrm{H}), 7.01(\mathrm{dd}, J=7.8,1.3 \mathrm{~Hz}, 1 \mathrm{H}), 2.55(\mathrm{~s}, 3 \mathrm{H}) ;{ }^{13} \mathbf{C}$ NMR $(101$ MHz, DMSO-d 6 ) $\delta 199.0,161.6,139.7,139.2,137.4,135.1,134.3,132.2,130.9$, 129.9, 129.43, 129.39, 128.0, 118.8, 29.5; HRMS (ESI-TOF) Calcd for $\mathrm{C}_{17} \mathrm{H}_{15} \mathrm{~N}_{2} \mathrm{O}_{3} \mathrm{~S}_{2}[\mathrm{M}+\mathrm{H}]^{+}:$359.0519; found: 359.0521 .

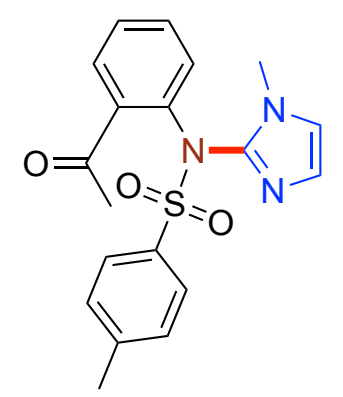


$N$-(2-acetylphenyl)-4-methyl- $N$-(1-methyl-1 $H$-imidazol-2-yl)benzenesulfonamide (2t):

Yellow solid; Eluent: petroleum ether/ethyl acetate 15:1; $100.8 \mathrm{mg}, 91 \%$; ${ }^{1} \mathbf{H}$ NMR $\left(400 \mathrm{MHz}, \mathrm{DMSO}-d_{6}\right) \delta 7.75(\mathrm{t}, \mathrm{J}=7.9 \mathrm{~Hz}, 1 \mathrm{H}), 7.55-7.10(\mathrm{~m}, 8 \mathrm{H}), 6.92-6.72(\mathrm{~m}$, 1H), 3.85 (s, 3H), 2.67 (s, 3H), 2.39 (s, 3H); ${ }^{13}$ C NMR (101 MHz, DMSO-d 6 ) $\delta 201.6$, $144.9,141.3,139.5,134.8,133.2,131.1,130.6,129.7,129.1,128.0,126.7,122.4$, 33.0, 30.7, 21.4; HRMS (ESI-TOF) Calcd for $\mathrm{C}_{19} \mathrm{H}_{20} \mathrm{~N}_{3} \mathrm{O}_{3} \mathrm{~S}[\mathrm{M}+\mathrm{H}]^{+}$: 370.1220; found: 370.1238 .

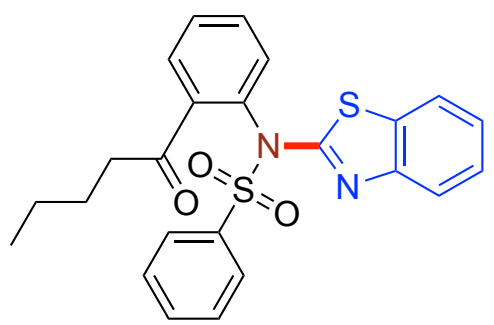

$N$-(benzo $[d]$ thiazol-2-yl)- $N$-(2-pentanoylphenyl)benzenesulfonamide (2u):

White solid; Eluent: petroleum ether/ethyl acetate 18:1; $120.2 \mathrm{mg}, 89 \%$; ${ }^{1} \mathbf{H}$ NMR $\left(400 \mathrm{MHz}, \mathrm{DMSO}-d_{6}\right) \delta 7.98-7.90(\mathrm{~m}, 4 \mathrm{H}), 7.78(\mathrm{tt}, J=7.0,1.2 \mathrm{~Hz}, 1 \mathrm{H}), 7.71-$ $7.61(\mathrm{~m}, 5 \mathrm{H}), 7.39(\mathrm{td}, J=8.2,7.8,1.3 \mathrm{~Hz}, 1 \mathrm{H}), 7.30(\mathrm{td}, J=7.8,1.2 \mathrm{~Hz}, 1 \mathrm{H}), 7.14$ $(\mathrm{dd}, J=7.6,1.4 \mathrm{~Hz}, 1 \mathrm{H}), 2.91(\mathrm{brs}, 2 \mathrm{H}), 1.52-1.40(\mathrm{~m}, 2 \mathrm{H}), 1.28-1.17(\mathrm{~m}, 2 \mathrm{H})$, $0.79(\mathrm{t}, J=7.4 \mathrm{~Hz}, 3 \mathrm{H}) ;{ }^{13} \mathrm{C}$ NMR (101 MHz, DMSO- $\left.d_{6}\right) \delta 201.1,161.6,149.5$, $139.5,137.5,134.7,134.4,132.5,132.3,131.4,130.5,129.6,129.5,128.2,126.3$, 124.3, 121.6, 121.3, 40.5, 25.4, 21.5, 13.7; HRMS (ESI-TOF) Calcd for $\mathrm{C}_{24} \mathrm{H}_{23} \mathrm{~N}_{2} \mathrm{O}_{3} \mathrm{~S}_{2}[\mathrm{M}+\mathrm{H}]^{+}:$451.1145; found: 451.1173 . 


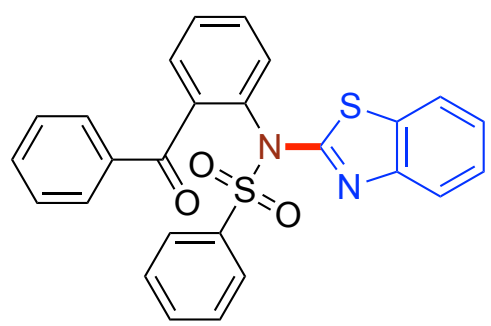

$N$-(benzo[d]thiazol-2-yl)- $N$-(2-benzoylphenyl)benzenesulfonamide (2v):

White solid; Eluent: petroleum ether/ethyl acetate $18: 1 ; 124.1 \mathrm{mg}, 88 \%$; ${ }^{1} \mathbf{H}$ NMR $\left(400 \mathrm{MHz}, \mathrm{DMSO}-d_{6}\right) \delta 7.95-7.85(\mathrm{~m}, 3 \mathrm{H}), 7.78-7.66(\mathrm{~m}, 6 \mathrm{H}), 7.65-7.56(\mathrm{~m}, 4 \mathrm{H})$, $7.47-7.36(\mathrm{~m}, 3 \mathrm{H}), 7.31(\mathrm{td}, J=7.7,1.2 \mathrm{~Hz}, 1 \mathrm{H}), 7.27-7.22(\mathrm{~m}, 1 \mathrm{H}) ;{ }^{13} \mathbf{C} \mathbf{N M R}$ $\left(101 \mathrm{MHz}, \mathrm{DMSO}-d_{6}\right) \delta 194.4,162.0,149.8,139.5,137.8,136.9,136.1,135.2,133.9$, $133.2,132.6,132.0,131.0,130.4,130.3,130.0,128.8,128.7,126.8,124.9,122.0$, 121.9; HRMS (ESI-TOF) Calcd for $\mathrm{C}_{26} \mathrm{H}_{19} \mathrm{~N}_{2} \mathrm{O}_{3} \mathrm{~S}_{2}$ [M+H] $]^{+}$: 471.0832; found: 471.0838.

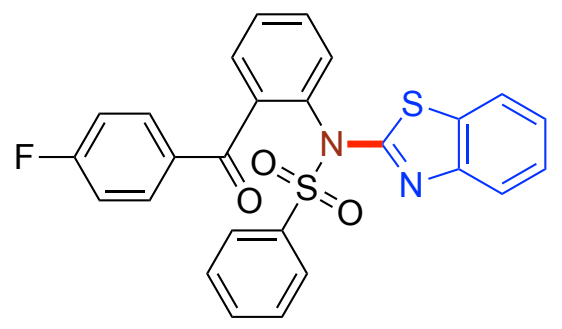

$N$-(benzo[d]thiazol-2-yl)- $N$-(2-(4-fluorobenzoyl)phenyl)benzenesulfonamide (2w): White solid; Eluent: petroleum ether/ethyl acetate 18:1; $121.5 \mathrm{mg}, 83 \%$; ${ }^{1} \mathbf{H}$ NMR $\left(400 \mathrm{MHz}, \mathrm{DMSO}-d_{6}\right) \delta 7.95-7.85(\mathrm{~m}, 3 \mathrm{H}), 7.78-7.58(\mathrm{~m}, 9 \mathrm{H}), 7.43-7.37(\mathrm{~m}$, 1H), $7.32(\mathrm{td}, J=8.0,1.2 \mathrm{~Hz}, 1 \mathrm{H}), 7.29-7.21(\mathrm{~m}, 3 \mathrm{H}) ;{ }^{13} \mathbf{C}$ NMR (101 MHz, DMSO- $\left.d_{6}\right) \delta 192.6,166.3,163.8,161.4,149.3,138.8,137.3,135.5,134.8,133.2(\mathrm{~d}, J$ $=2.9 \mathrm{~Hz}), 132.9,132.8,132.7,132.2,131.5,130.3,130.0,129.6,128.2,126.4,124.5$, 
121.6, 121.4, 115.6, 115.4; ${ }^{19}$ F NMR (376 MHz, DMSO-d $d_{6} \delta-105.3$; HRMS

(ESI-TOF) Calcd for $\mathrm{C}_{26} \mathrm{H}_{18} \mathrm{~N}_{2} \mathrm{O}_{3} \mathrm{~S}_{2} \mathrm{~F}[\mathrm{M}+\mathrm{H}]^{+}$: 489.0737 ; found: 489.0766 .

\section{References}

[1] Youn, S. W.; Ko, T. Y.; Jang, Y. H. Palladium-catalyzed regioselective synthesis of 3-arylindoles from N-Ts-anilines and styrenes. Angew. Chem., Int. Ed. 2017, 56, 6636-6640.

[2] Shah, M.; Wells, G.; Bradshaw, T. D.; Laughton, C. A.; Stevens, M. F. G.; Westwell, A. D. Antitubercular properties of substituted hydroxycyclohexadienones. Lett. Drug Des. Discov. 2006, 3, 419-423.

[3] Zhu, Y.-P.; Lian, M.; Jia, F.-C.; Liu, M.-C.; Yuan, J.-J.; Gao, Q.-H.; Wu, A.-X. I2 promoted domino oxidative cyclization for one-pot synthesis of 2-acylbenzothiazoles via metal-free sp3 C-H functionalization. Chem. Commun. 2012, 48, 9086-9088.

[4] Sumrit, P.; Chuawong, P.; Nanok, T.; Duangthongyou, T.; Hormnirun, P. Aluminum complexes containing salicylbenzoxazole ligands and their application in the ring-opening polymerization of rac-lactide and $\varepsilon$-caprolactone. Dalton Trans. 2016, 45, 9250-9266.

[5] Zhou, J.; Hong, C.; Pan, C. The photo-controlled polymerization-induced self-assembly and reorganization process for fabrication of polymeric nanomaterials. Mater. Chem. Front. 2017, 1, 1200-1206. 
9 NMR-Spectra of 1, Catalysts and 2

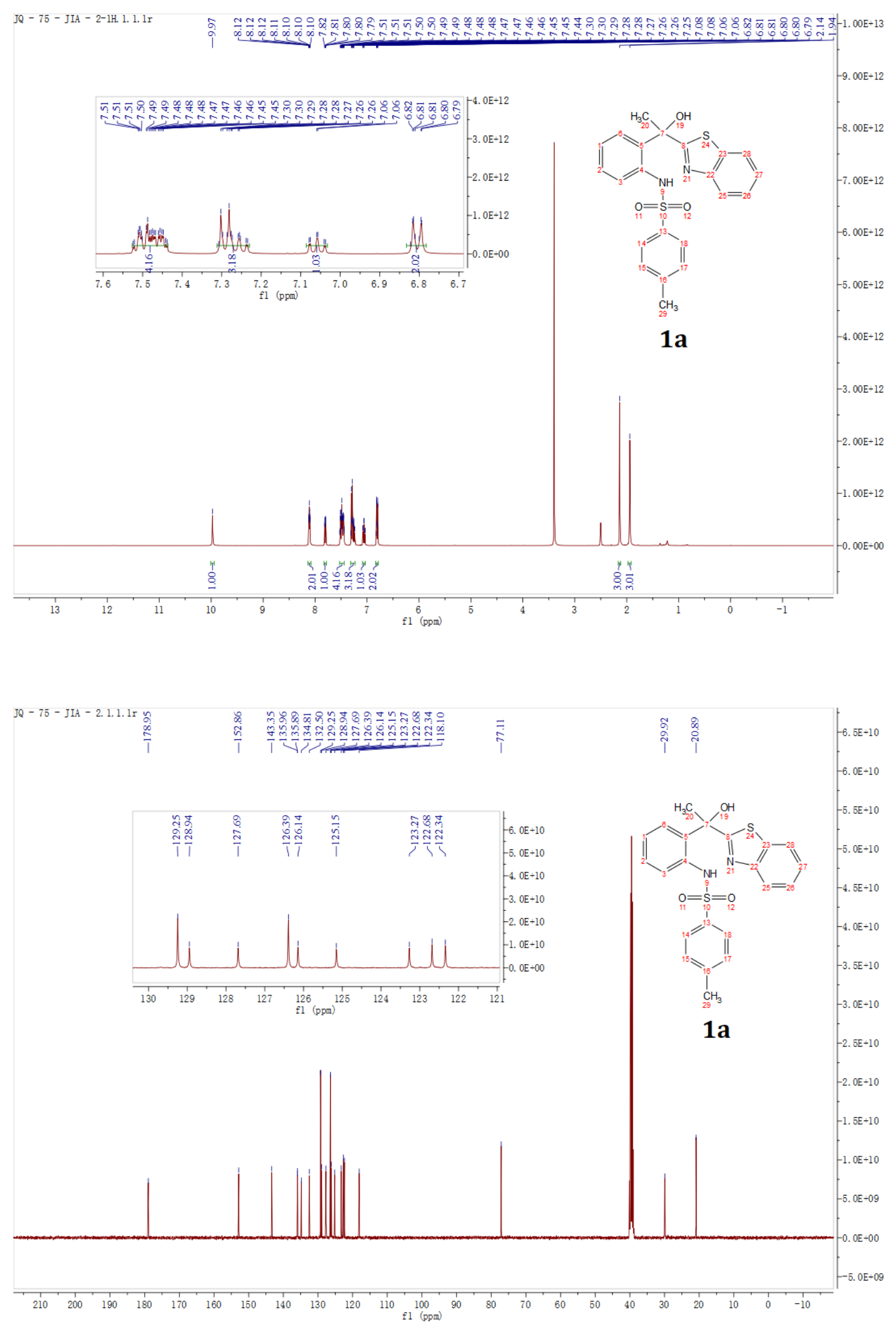



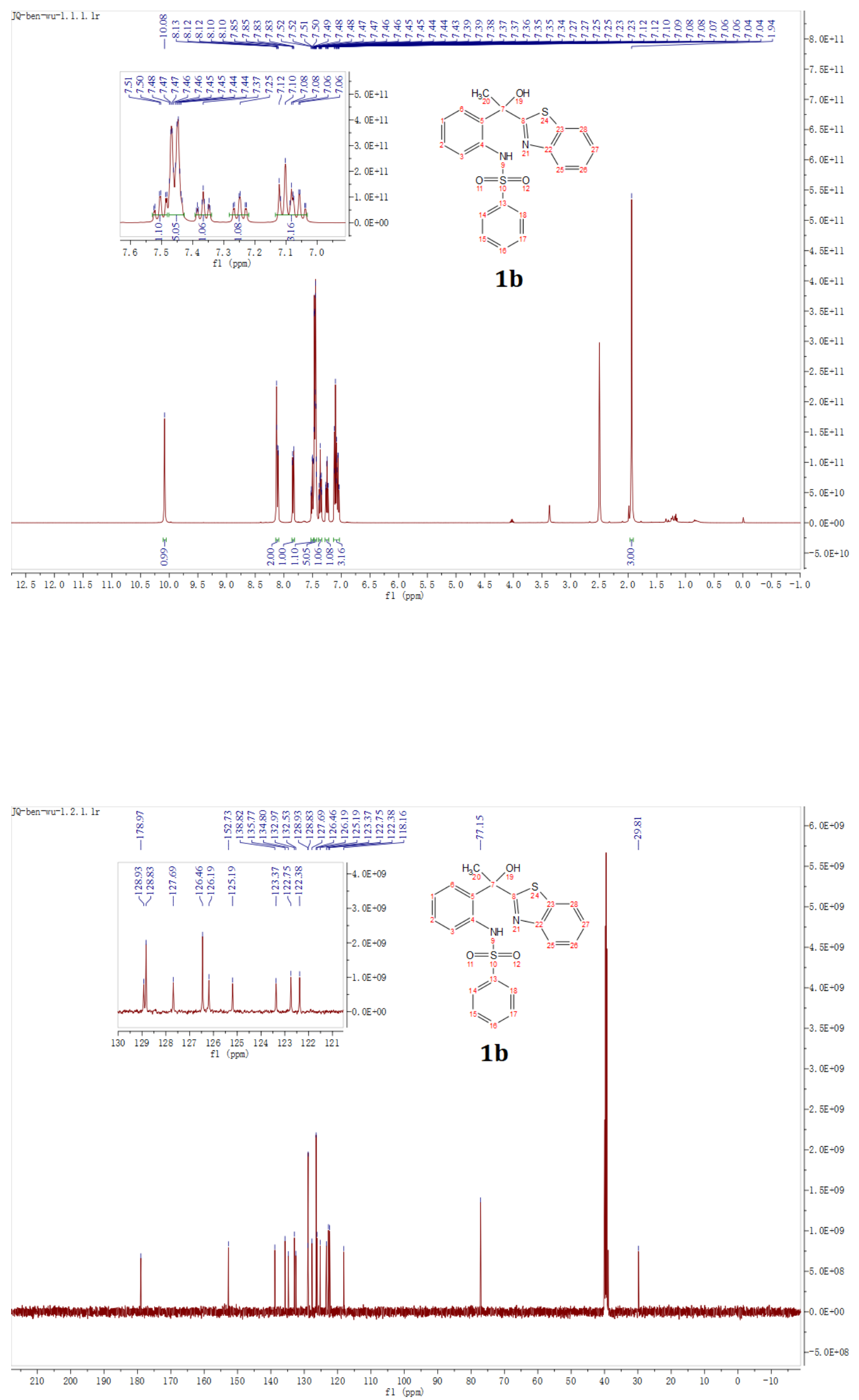

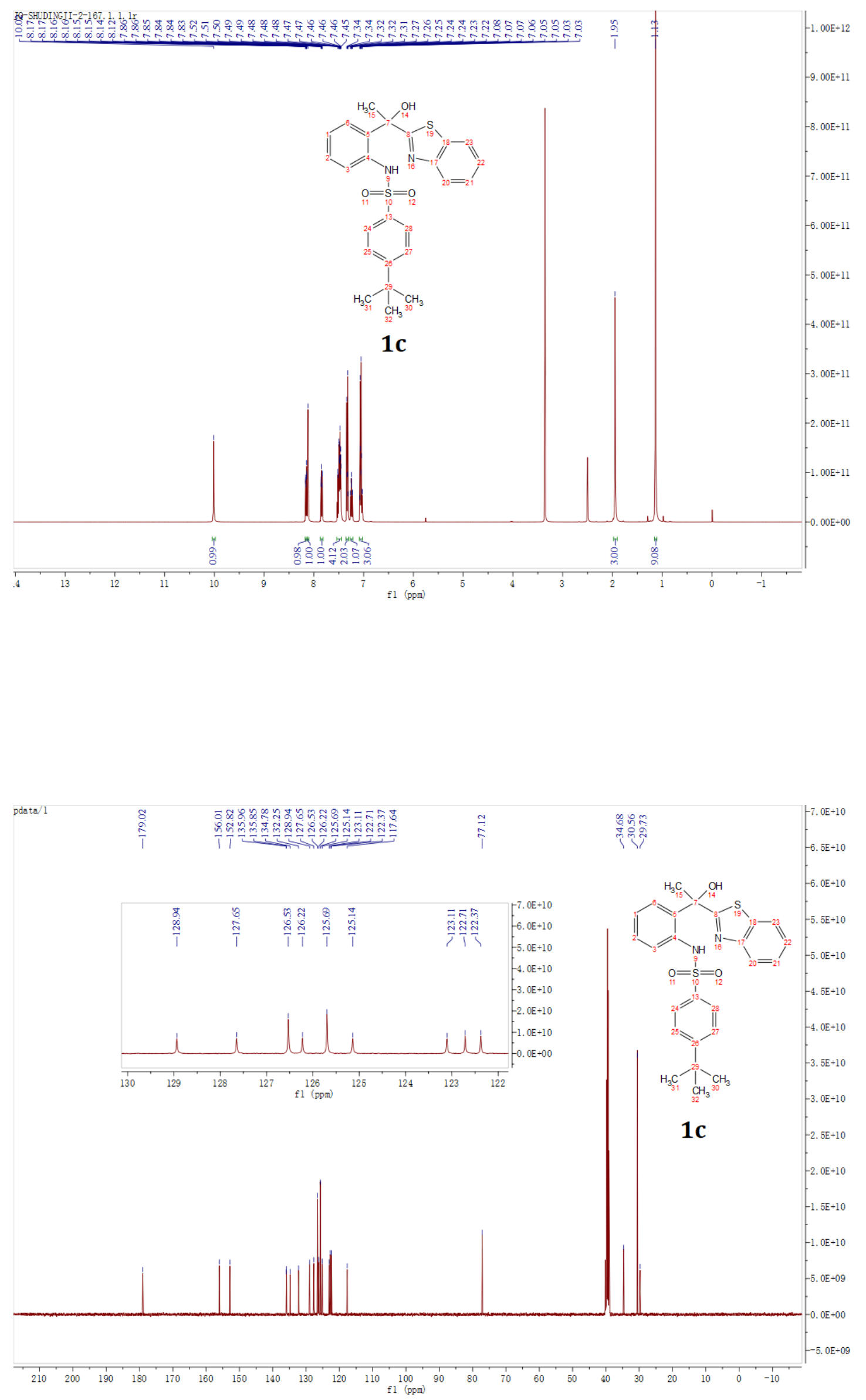

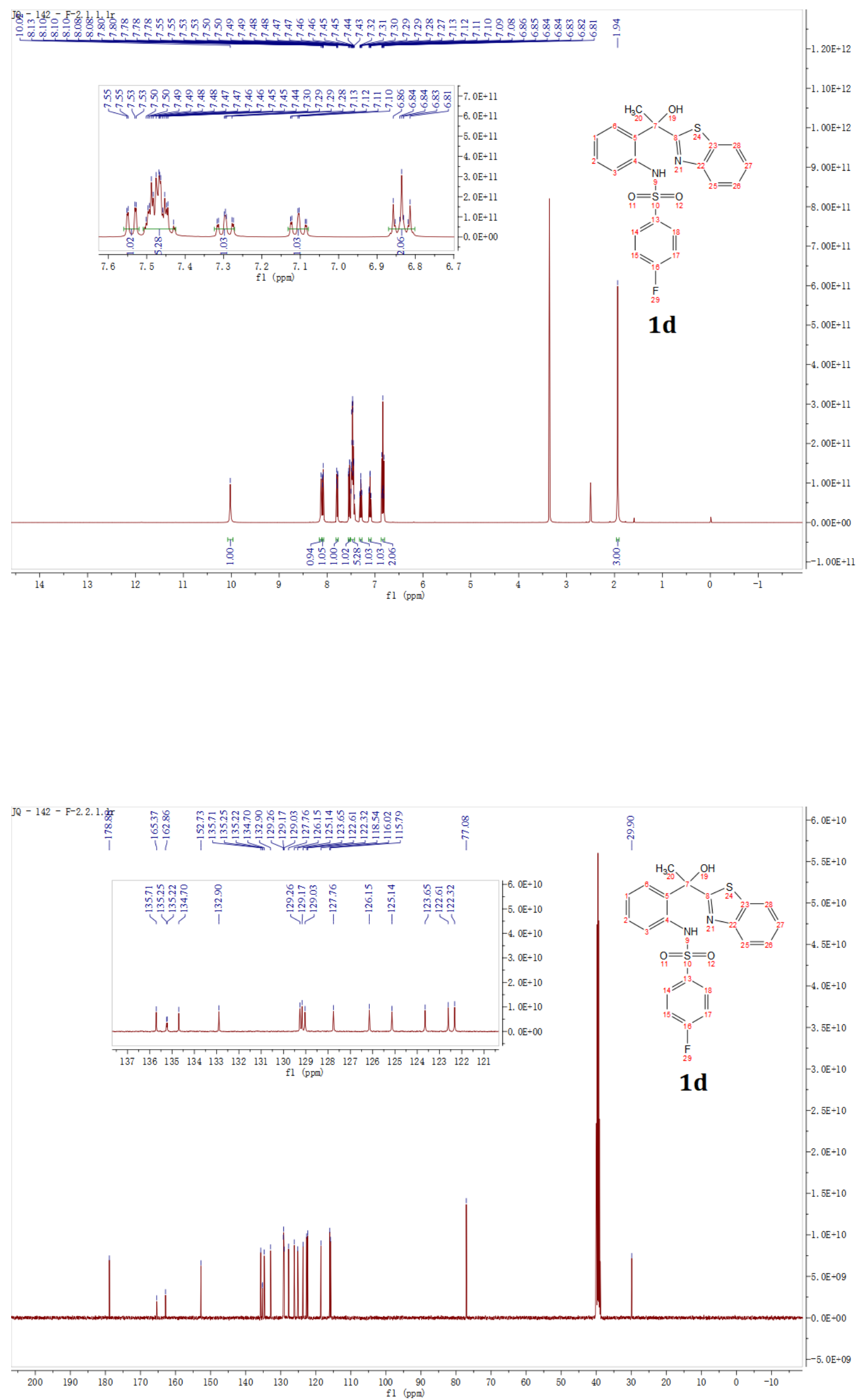

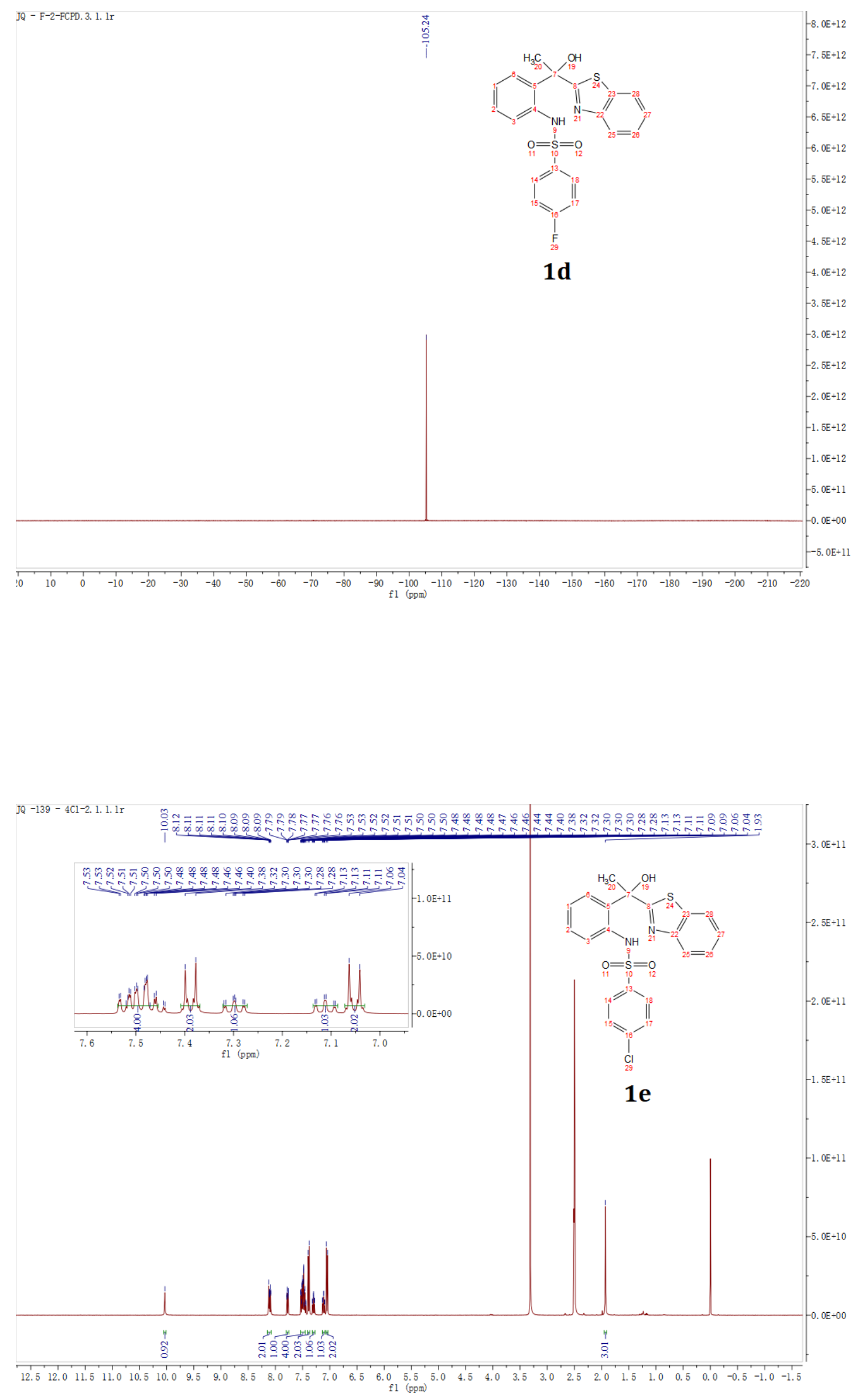

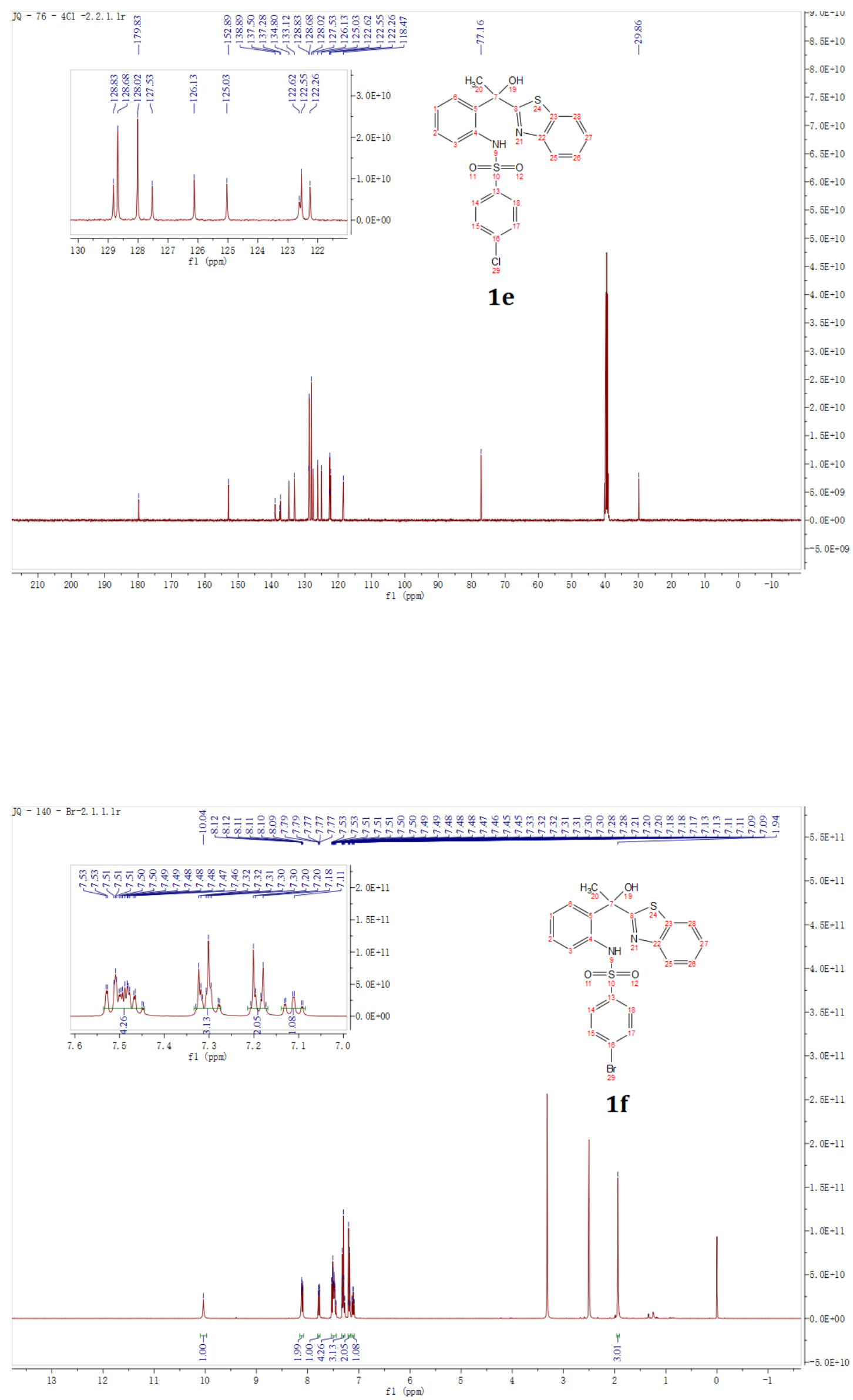

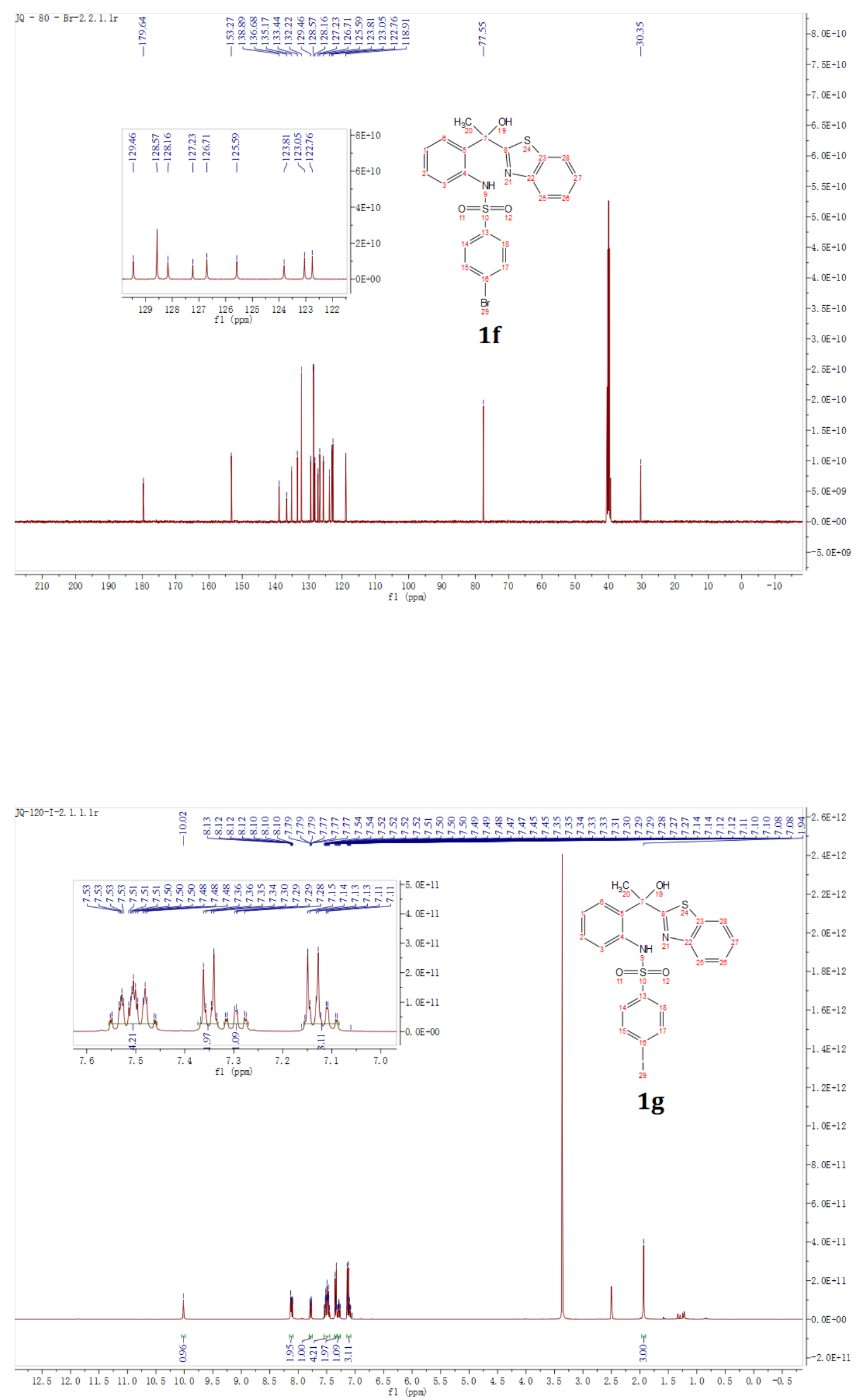

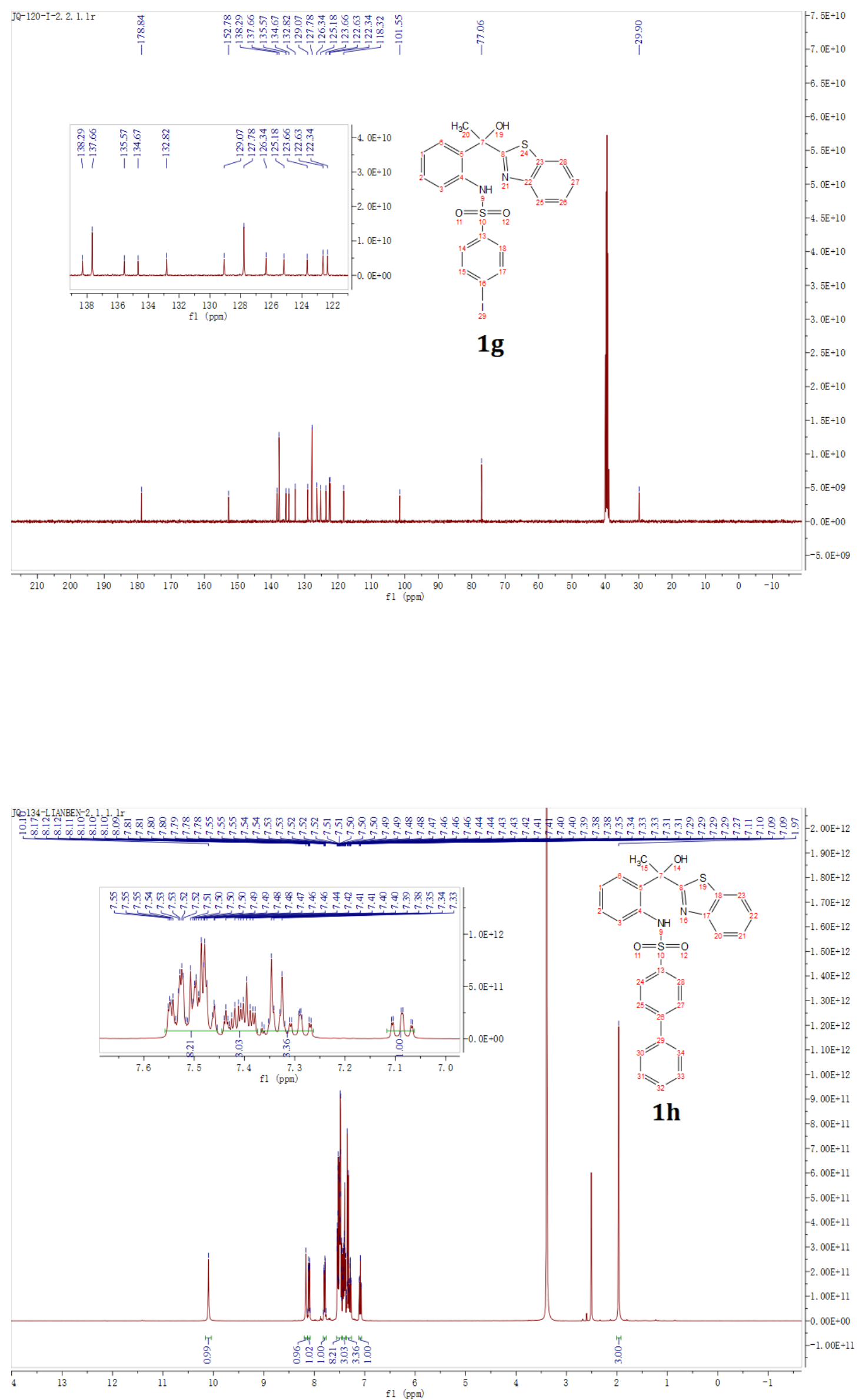

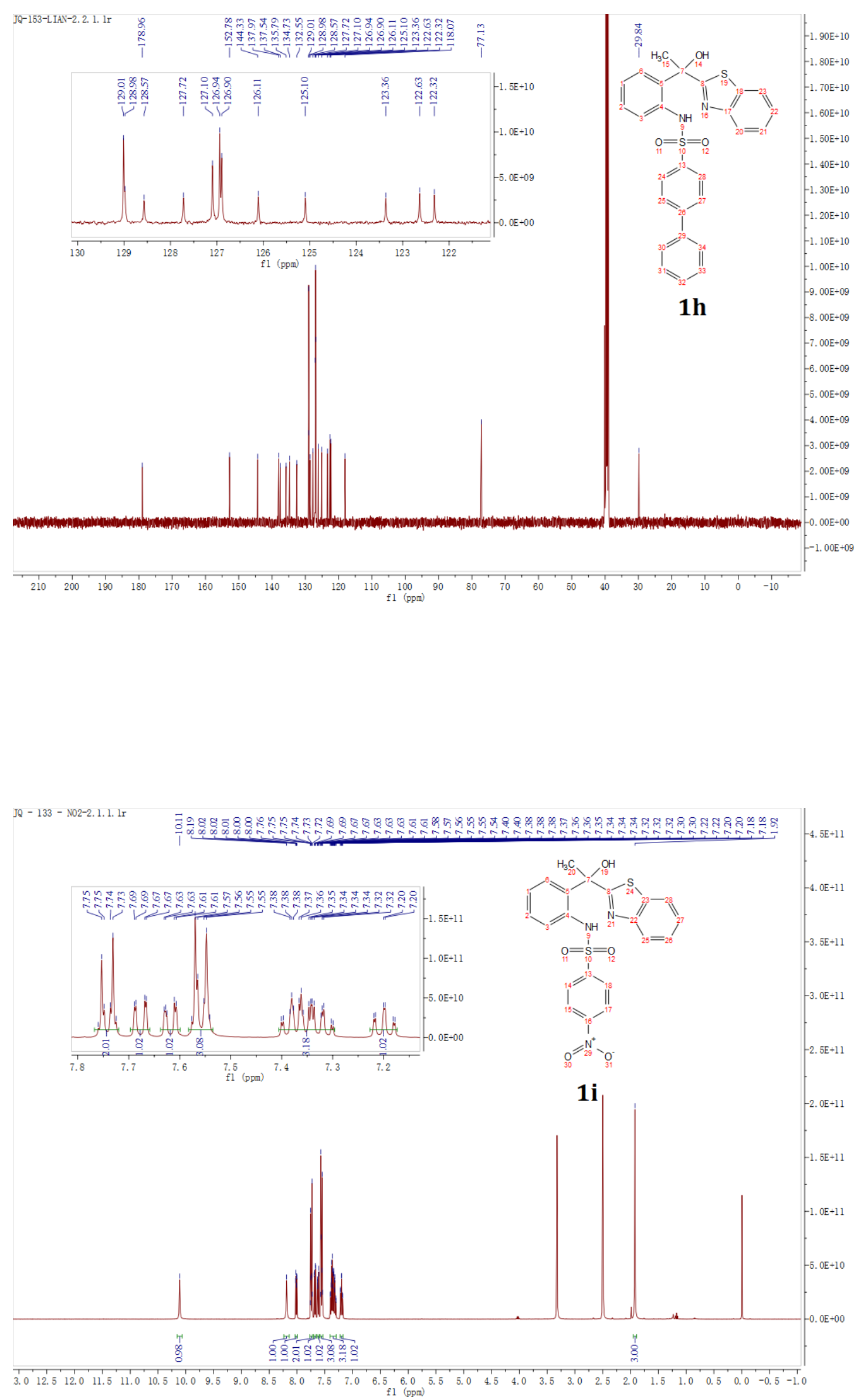

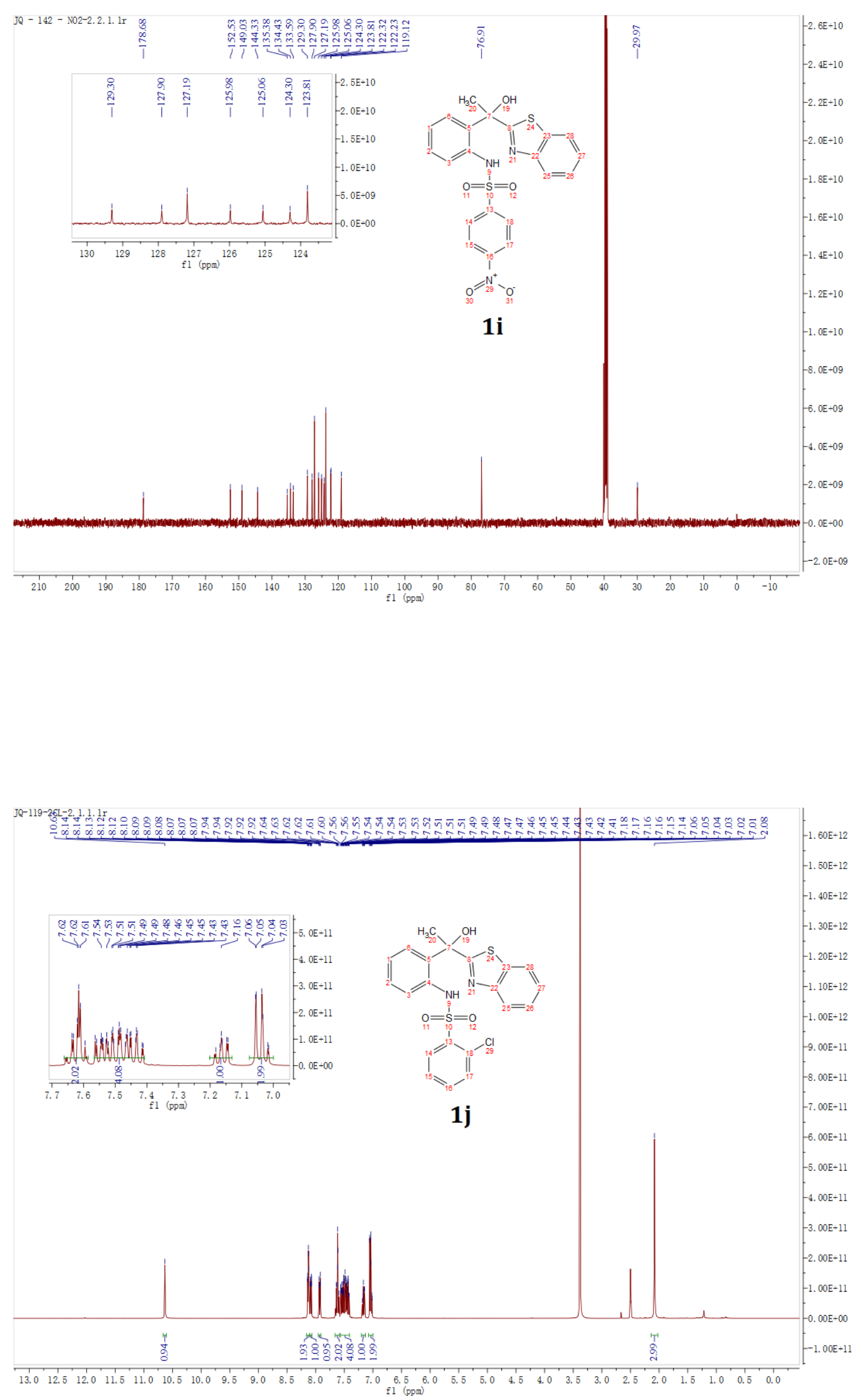

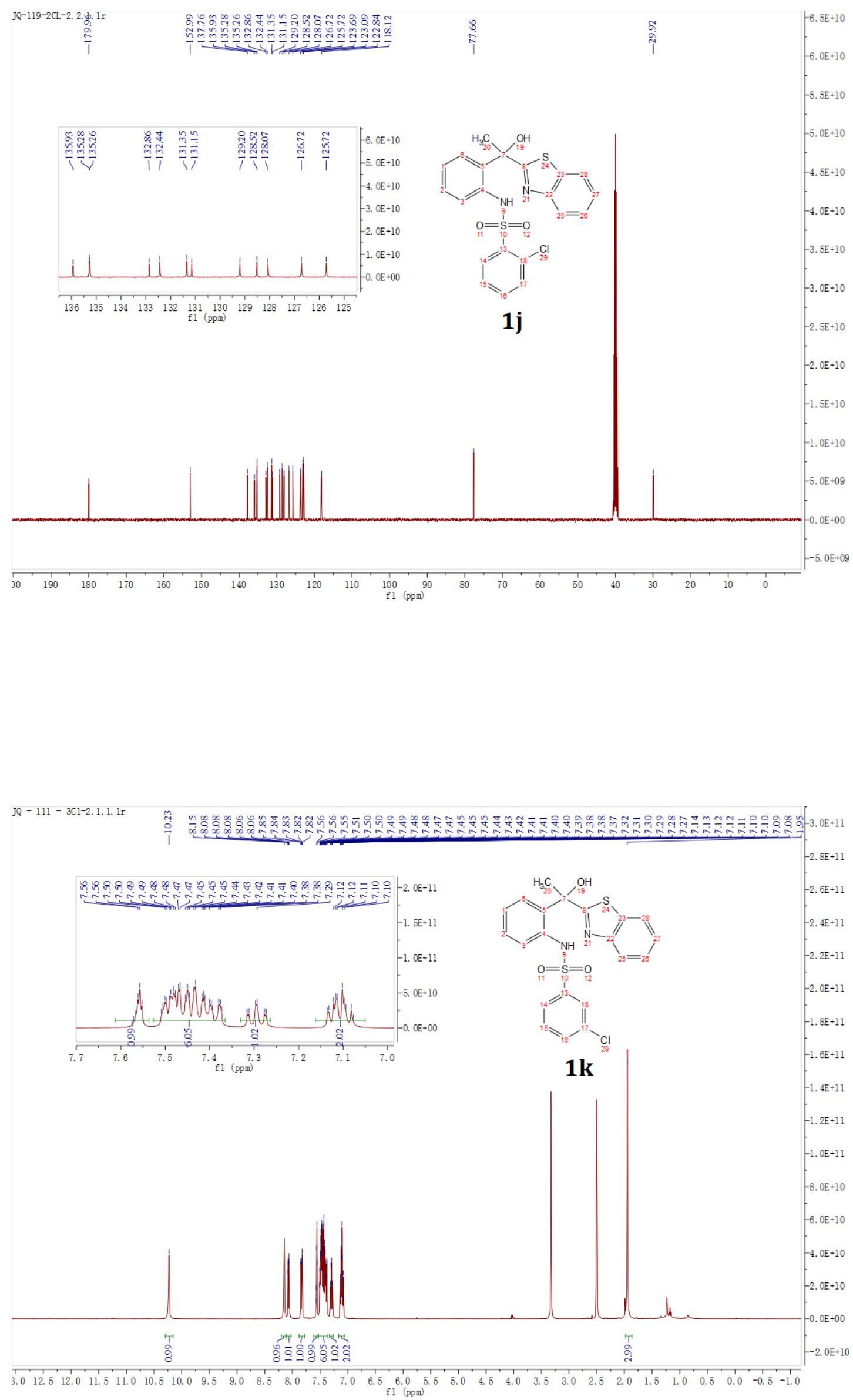

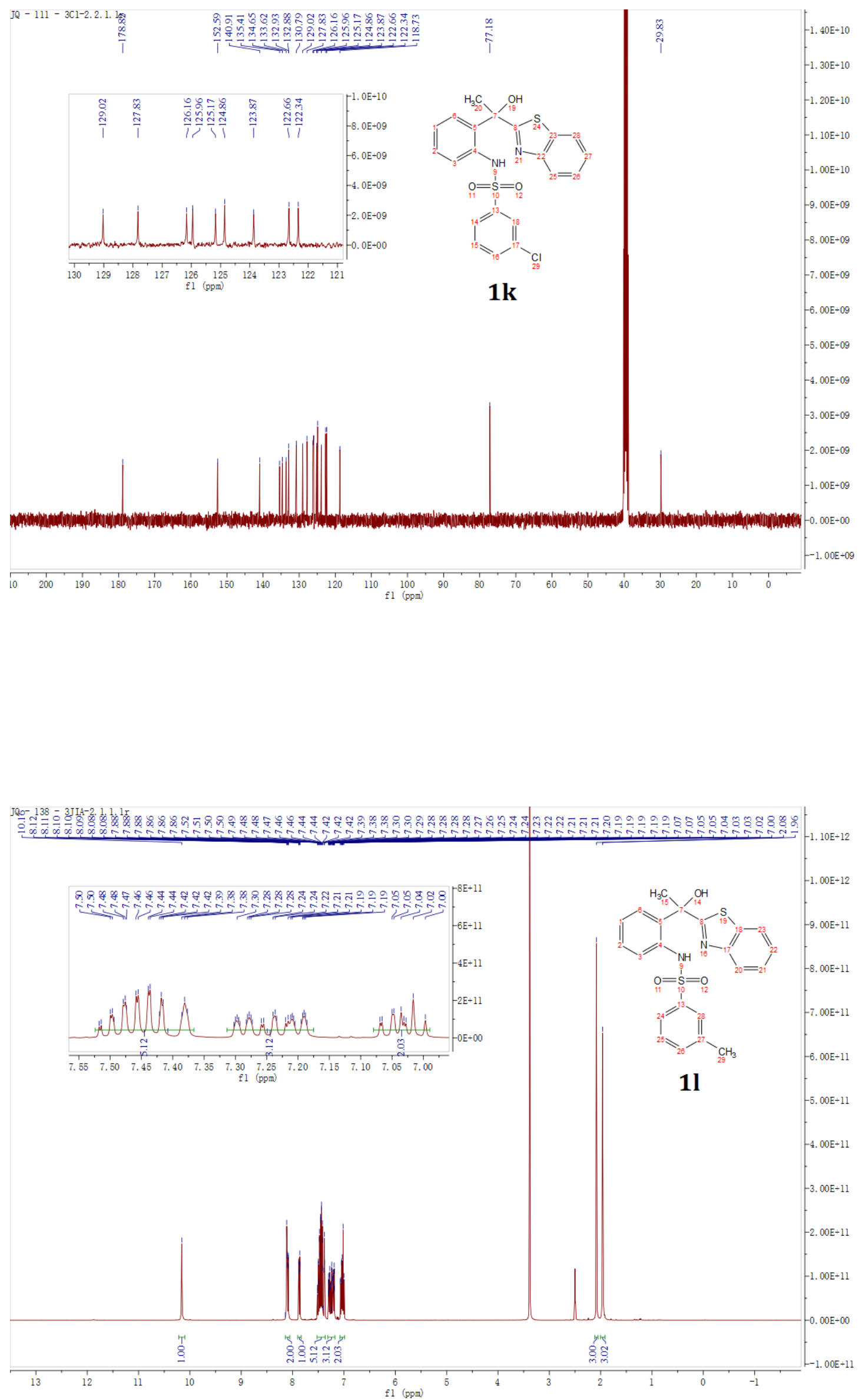


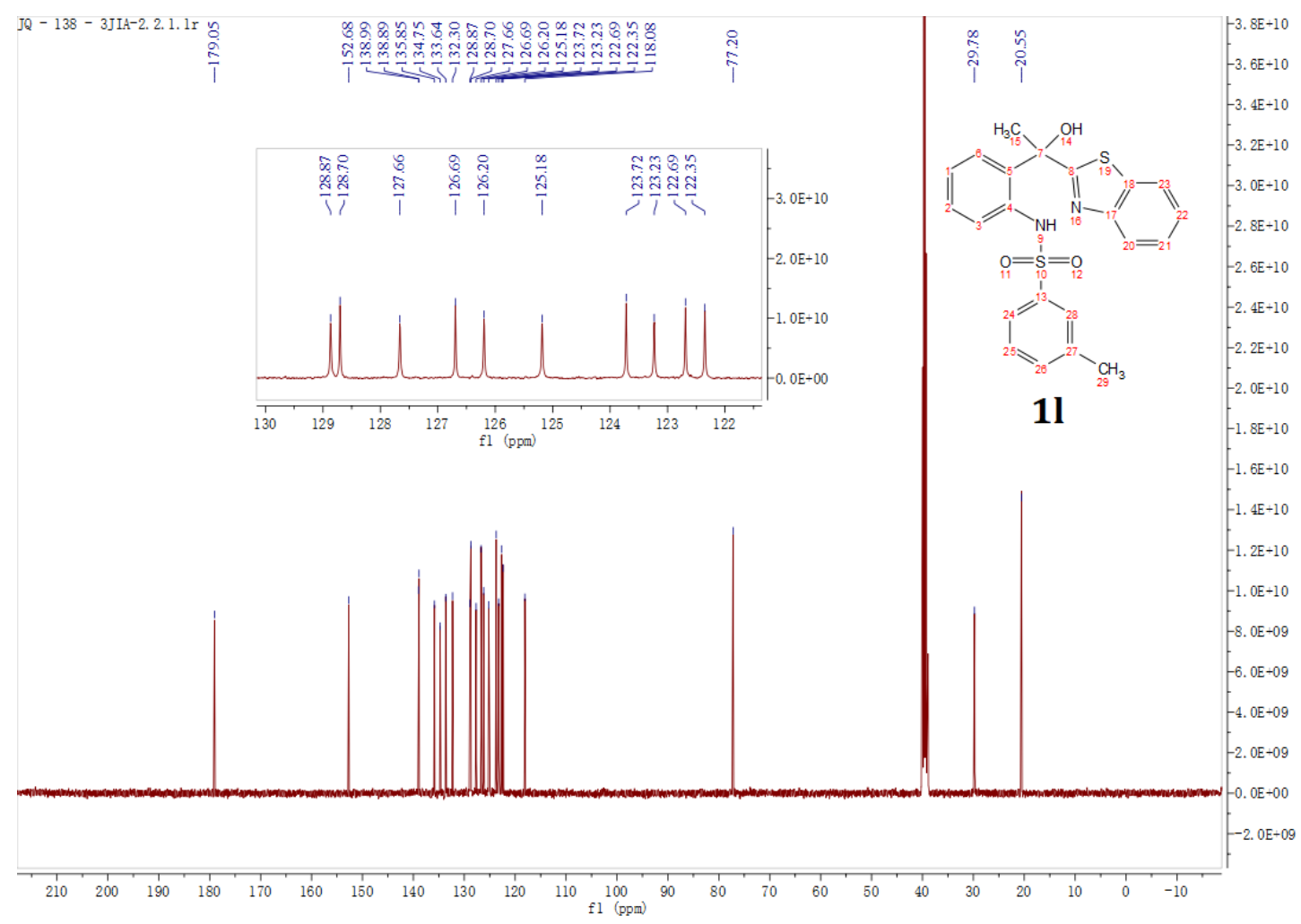

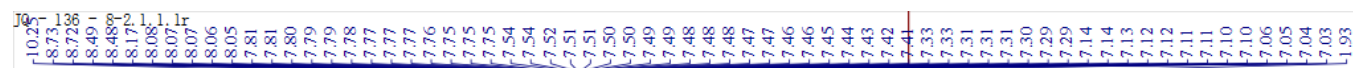
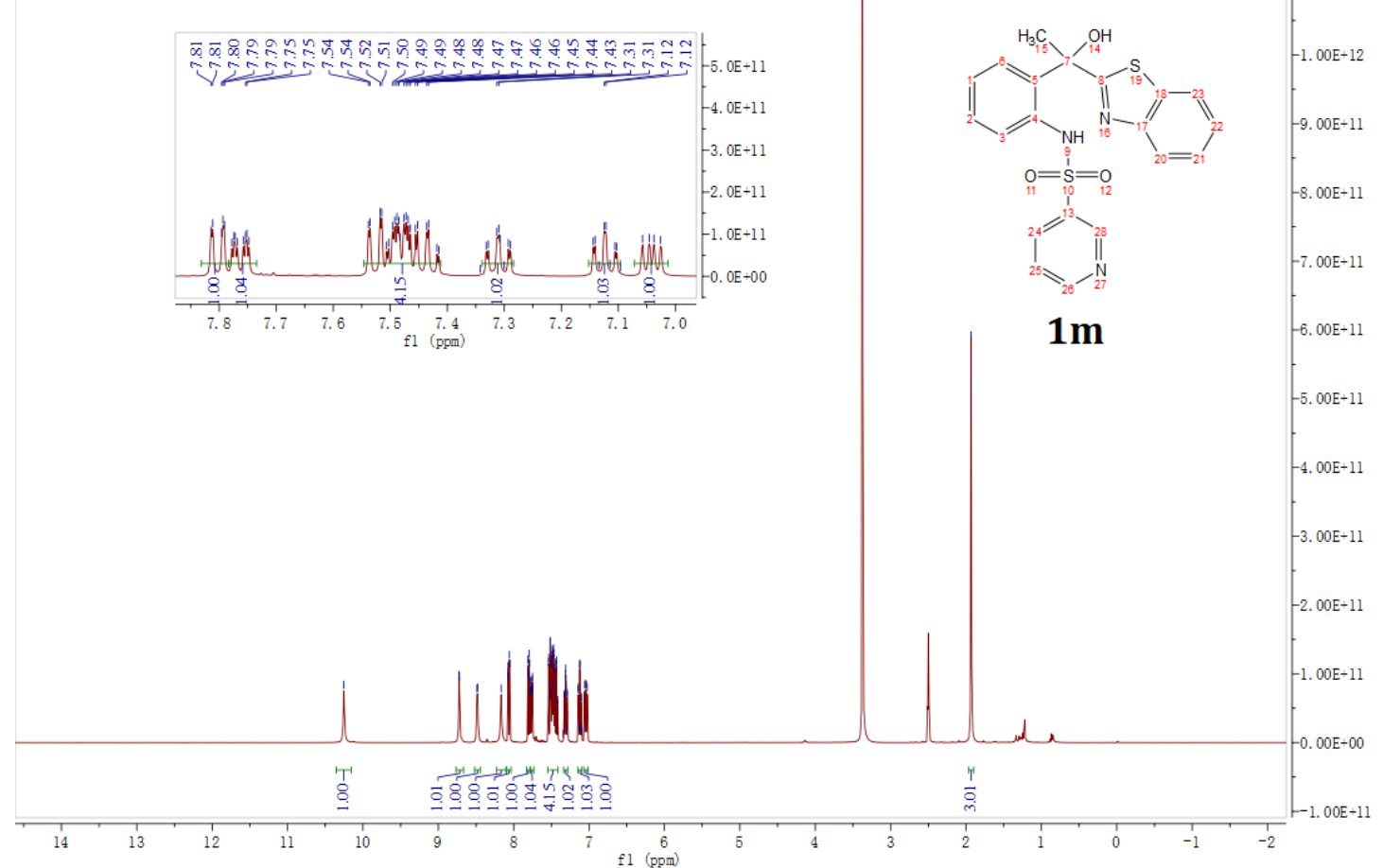

1. $10 \mathrm{E}+12$ 

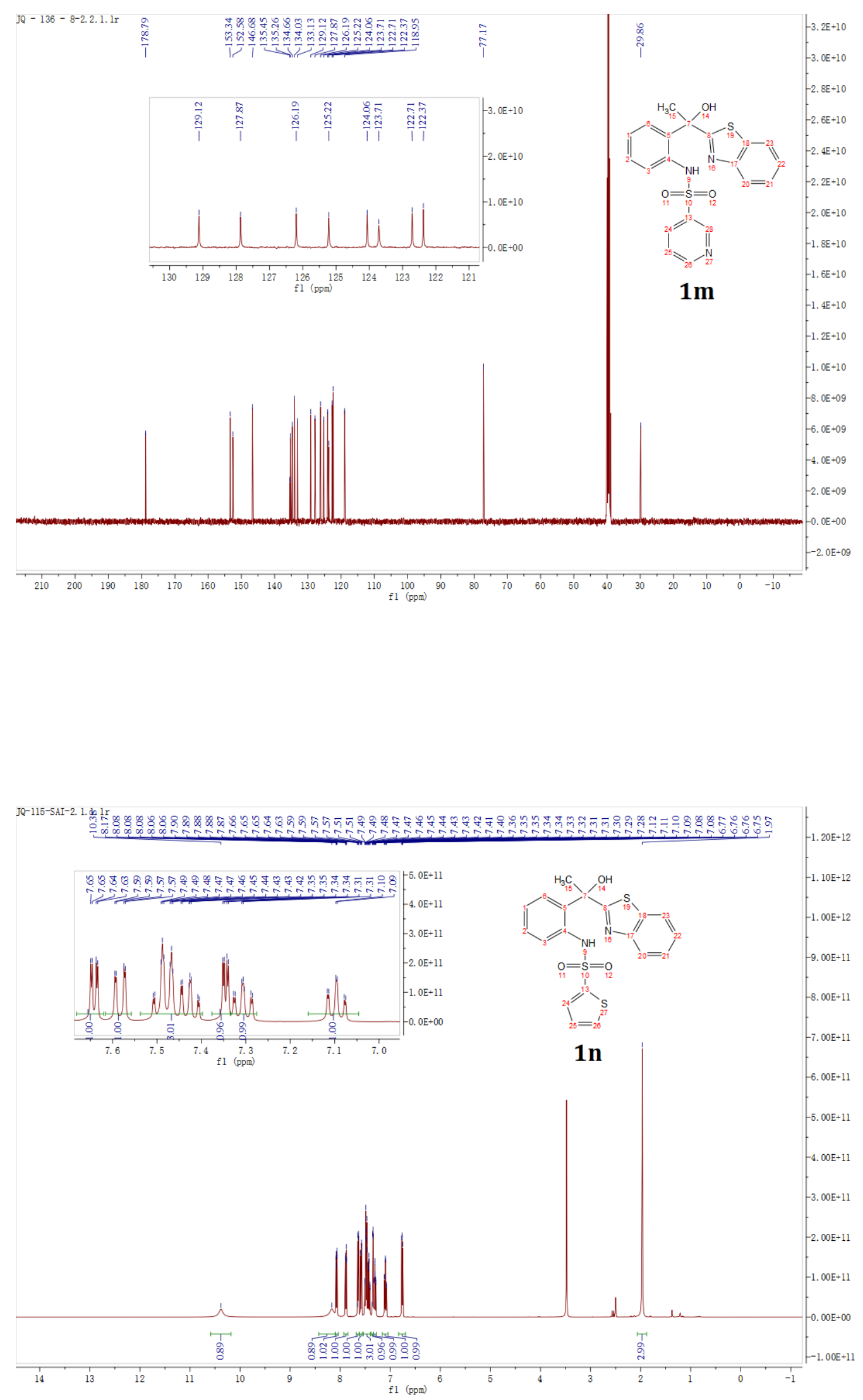

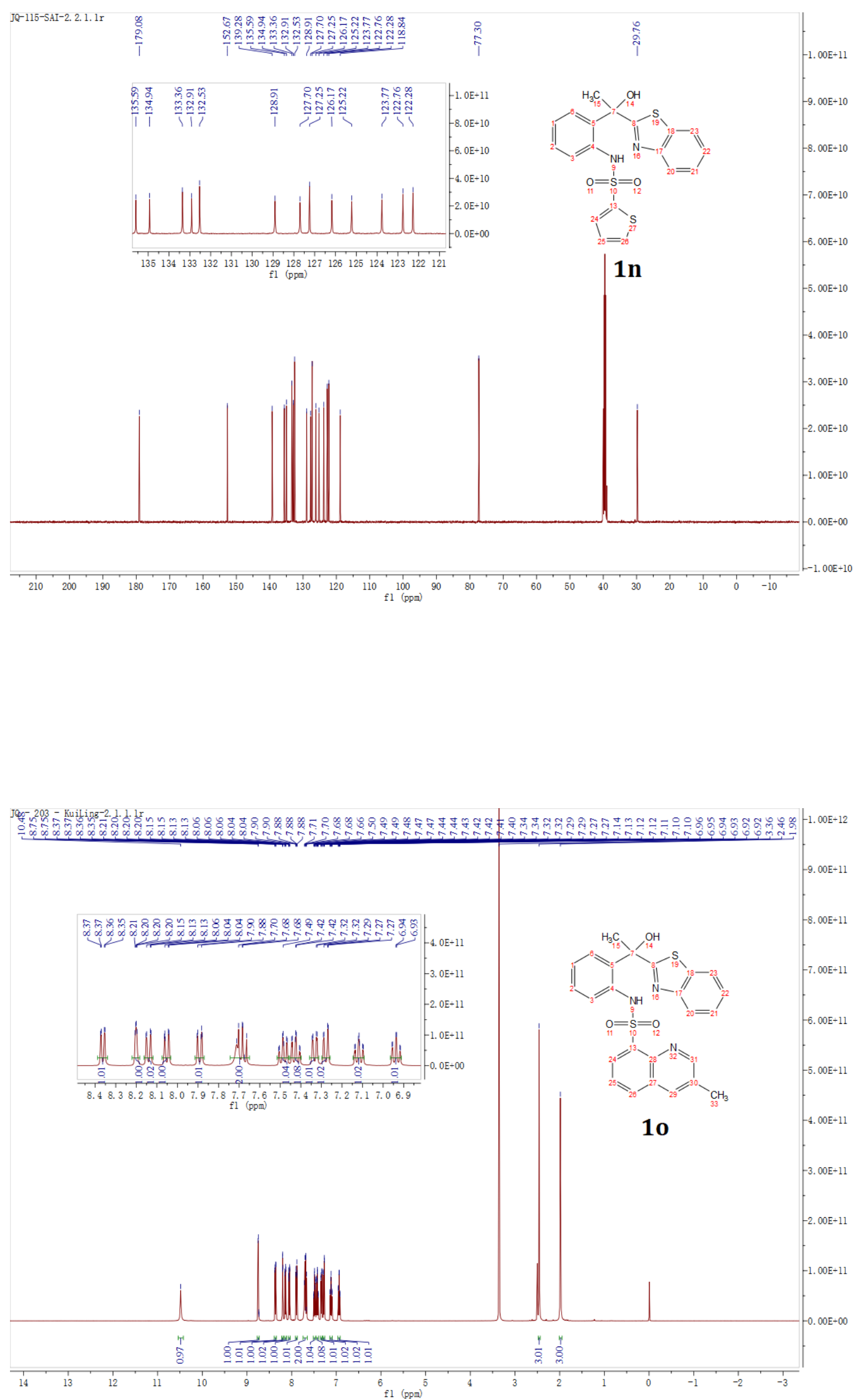

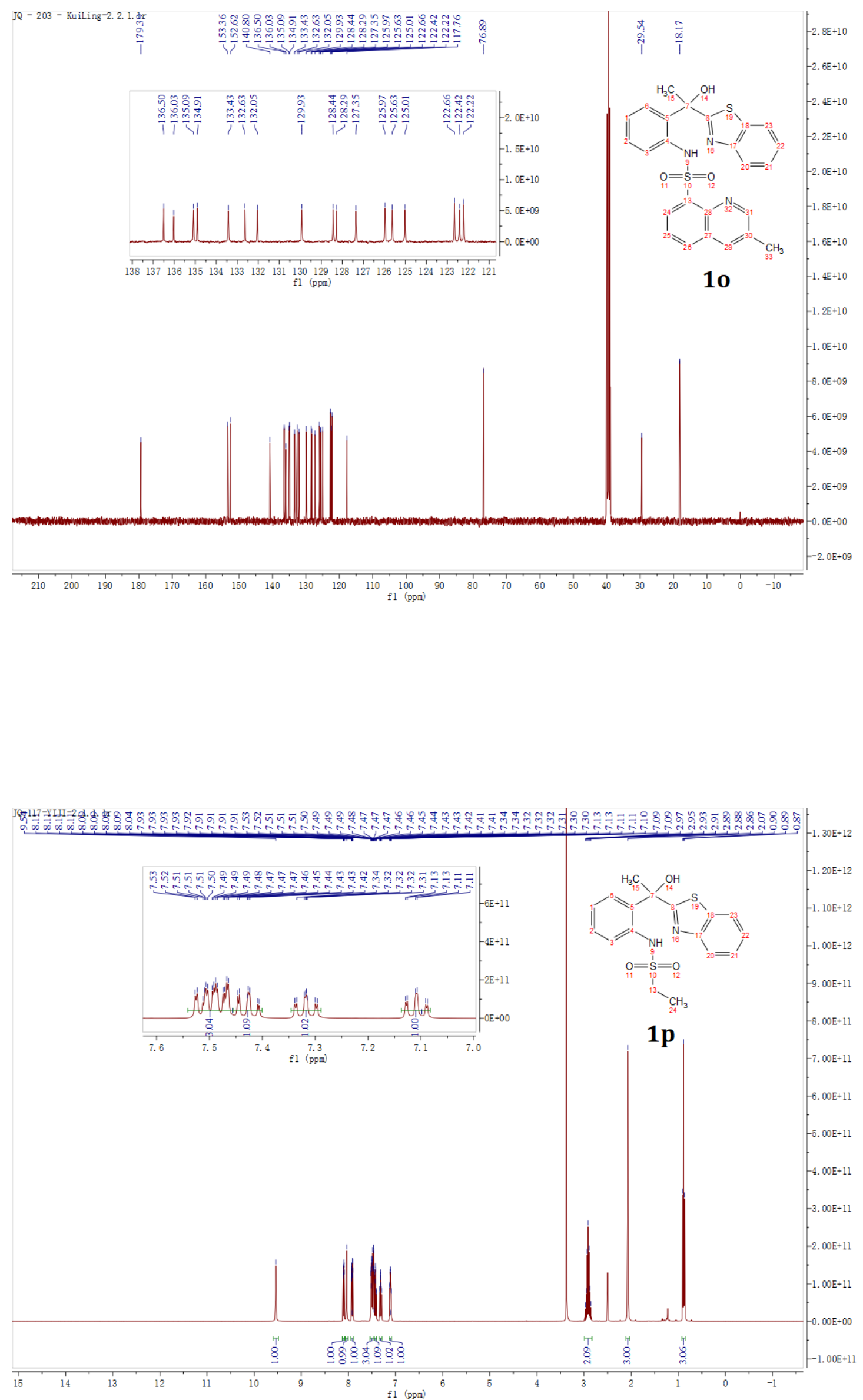


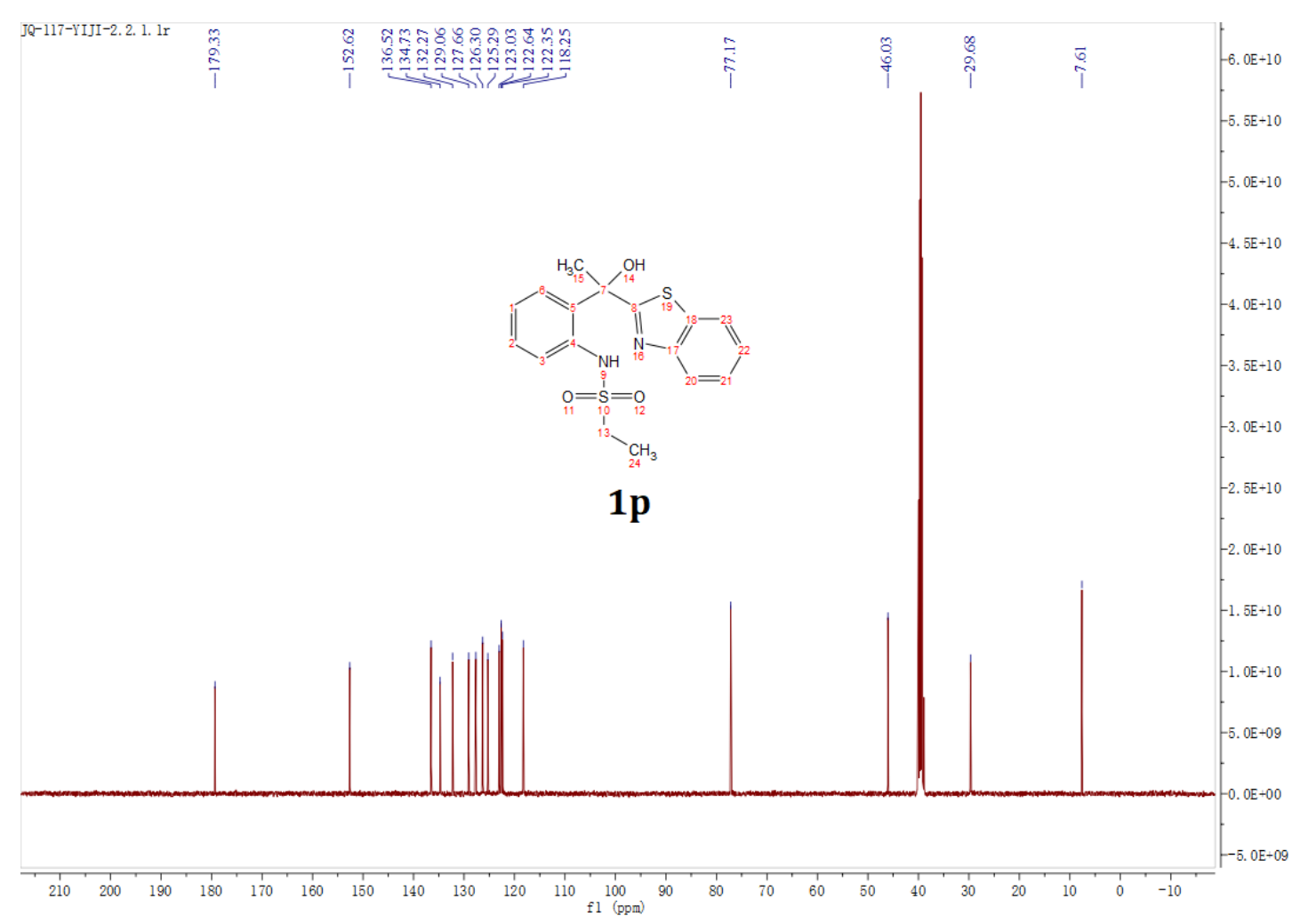

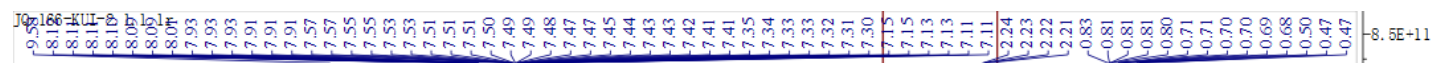
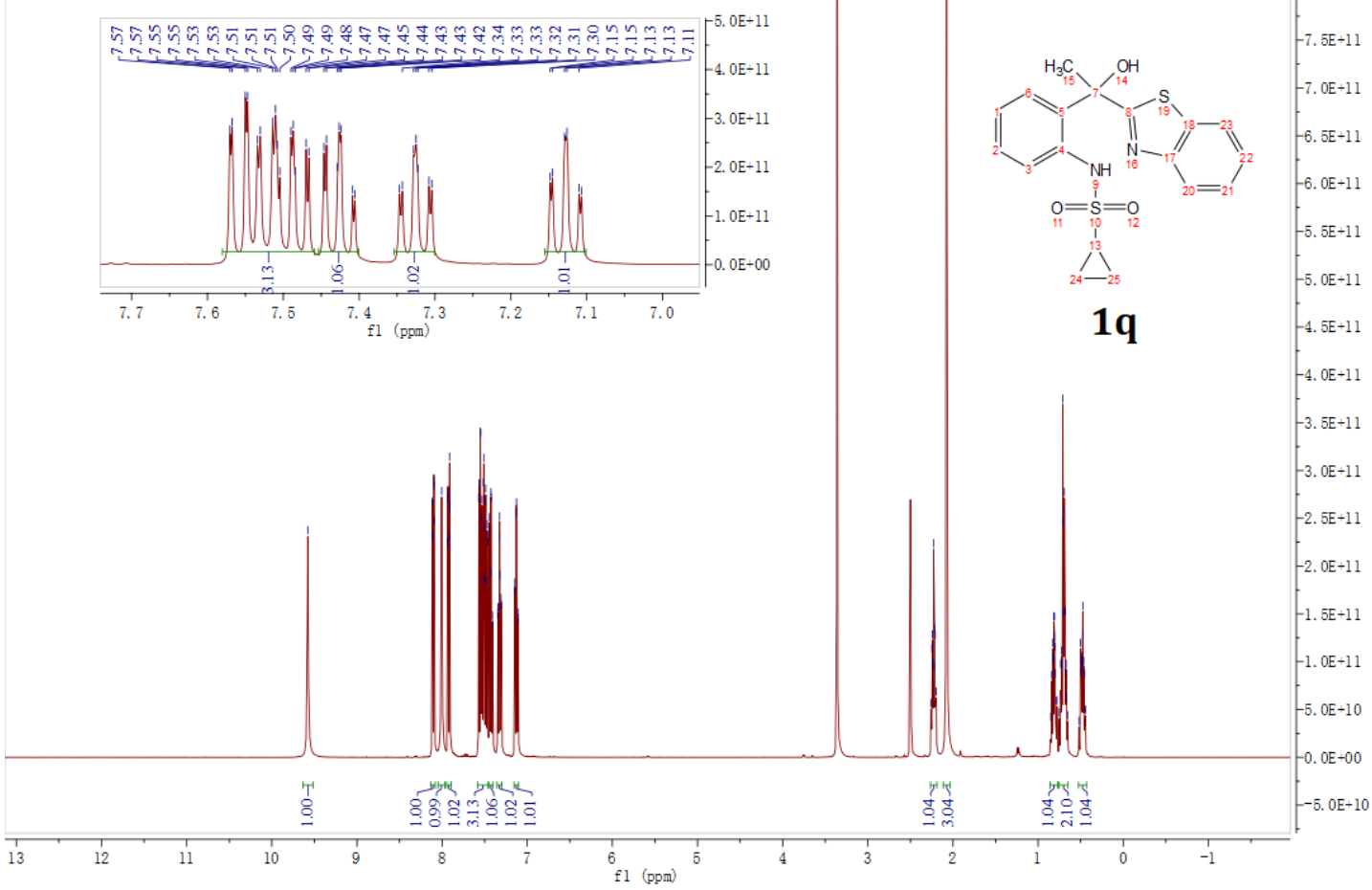

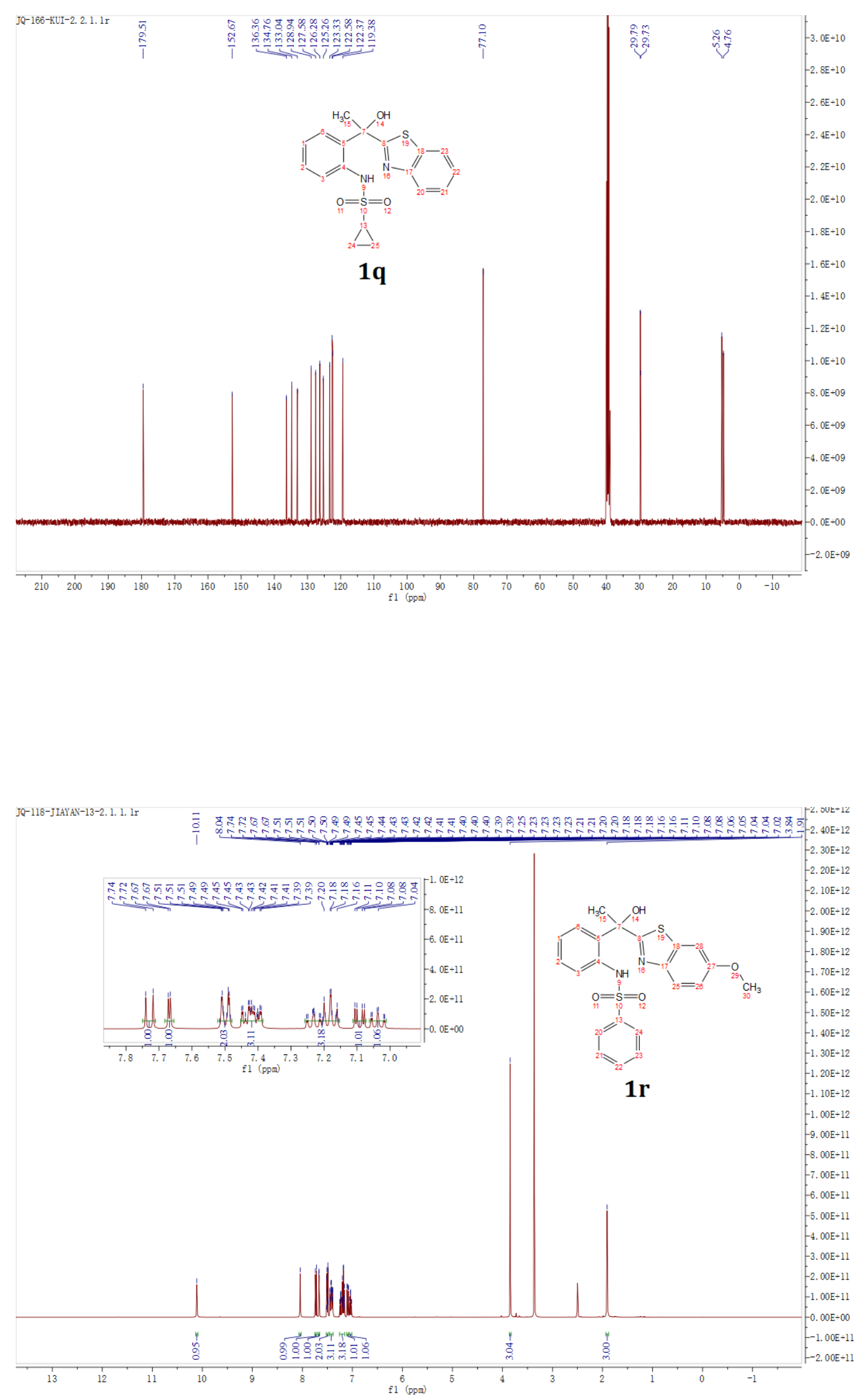

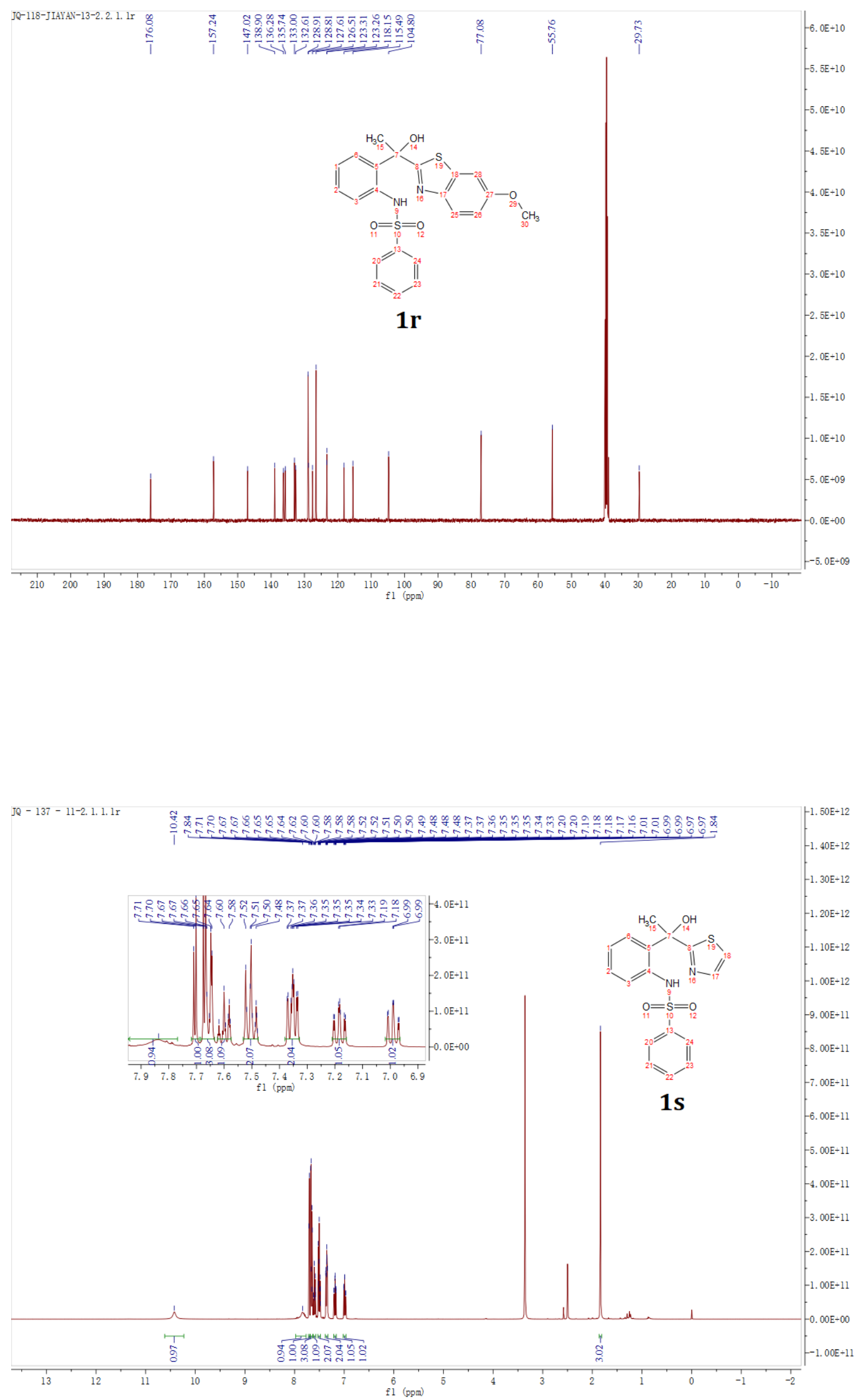

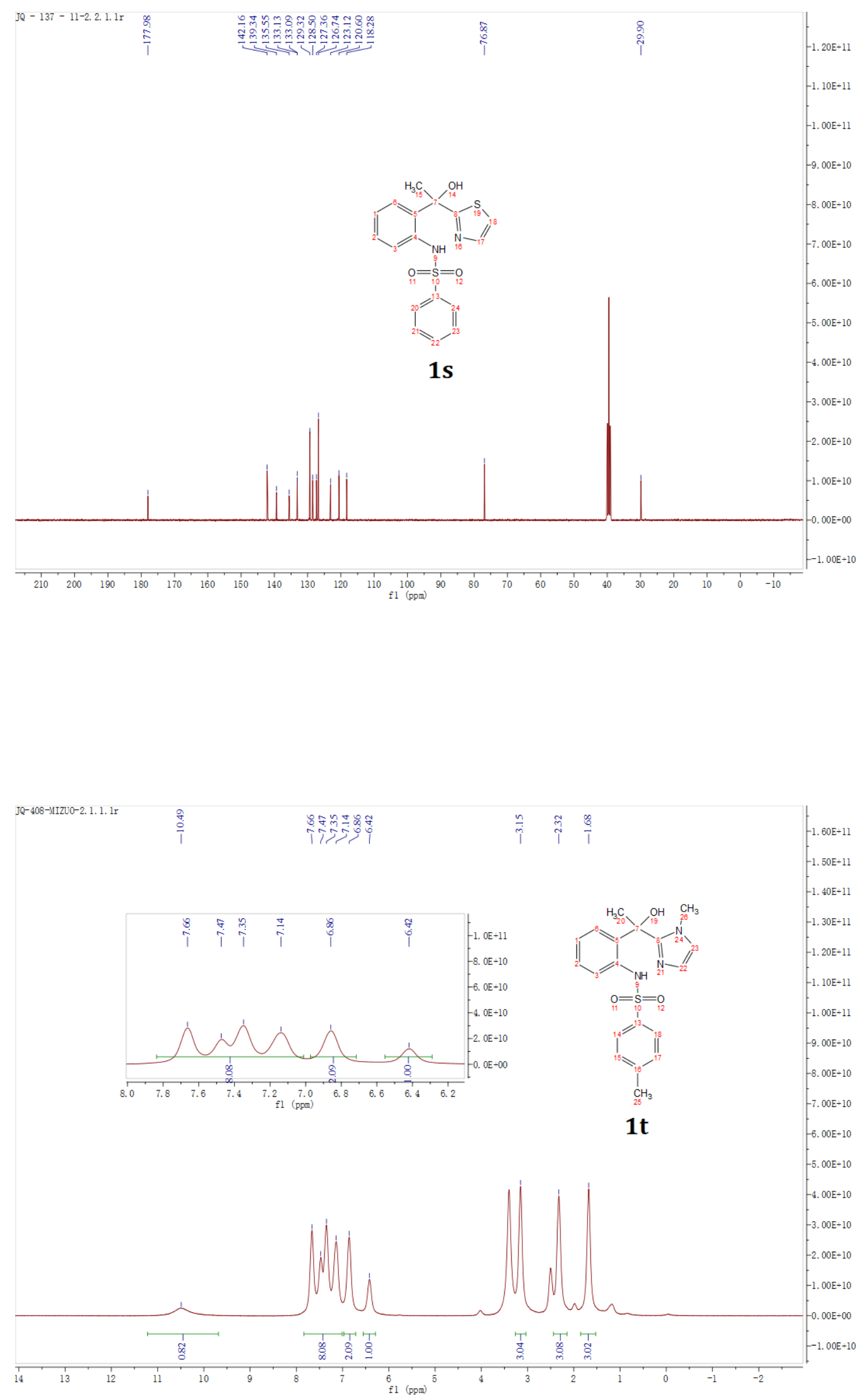

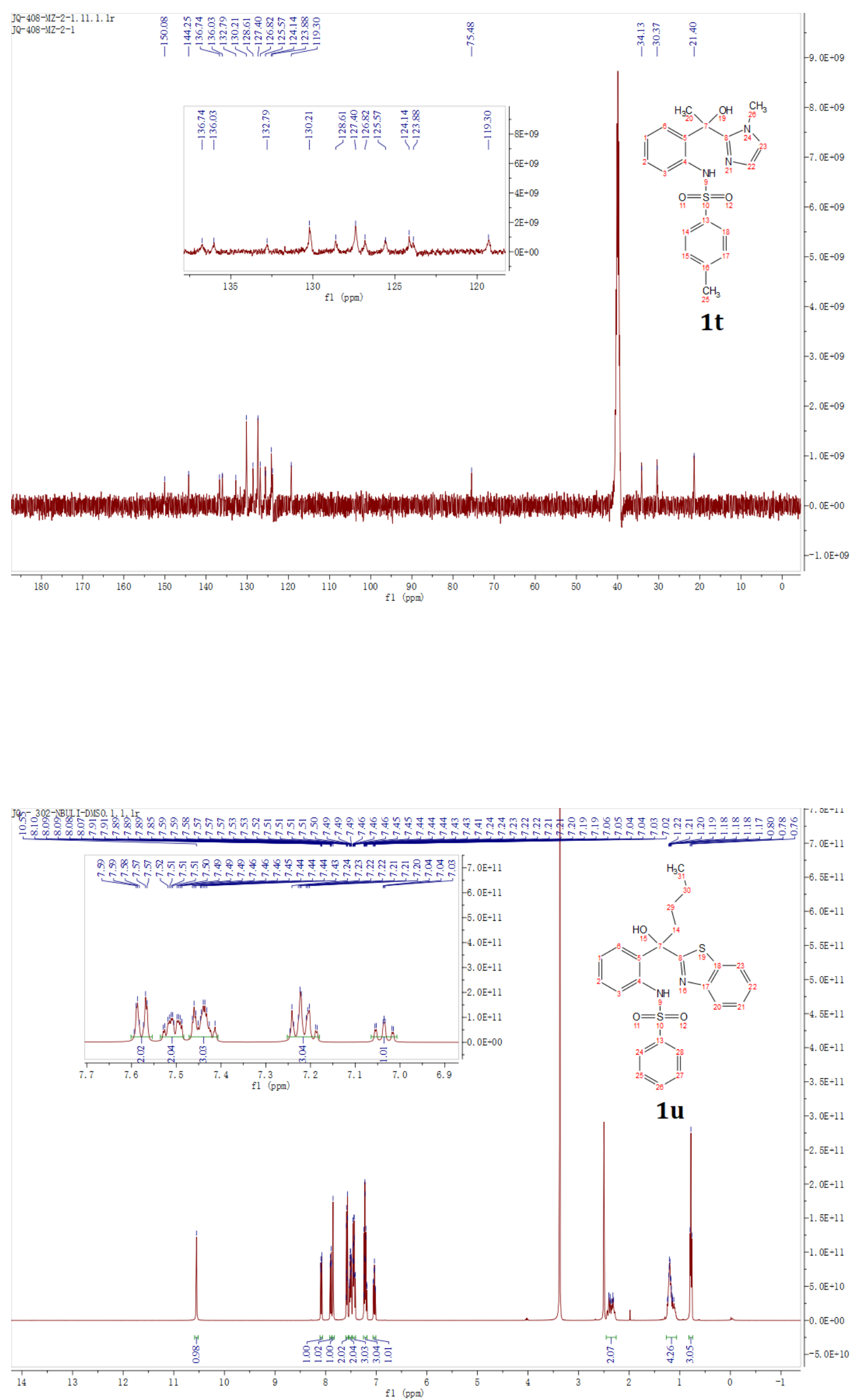

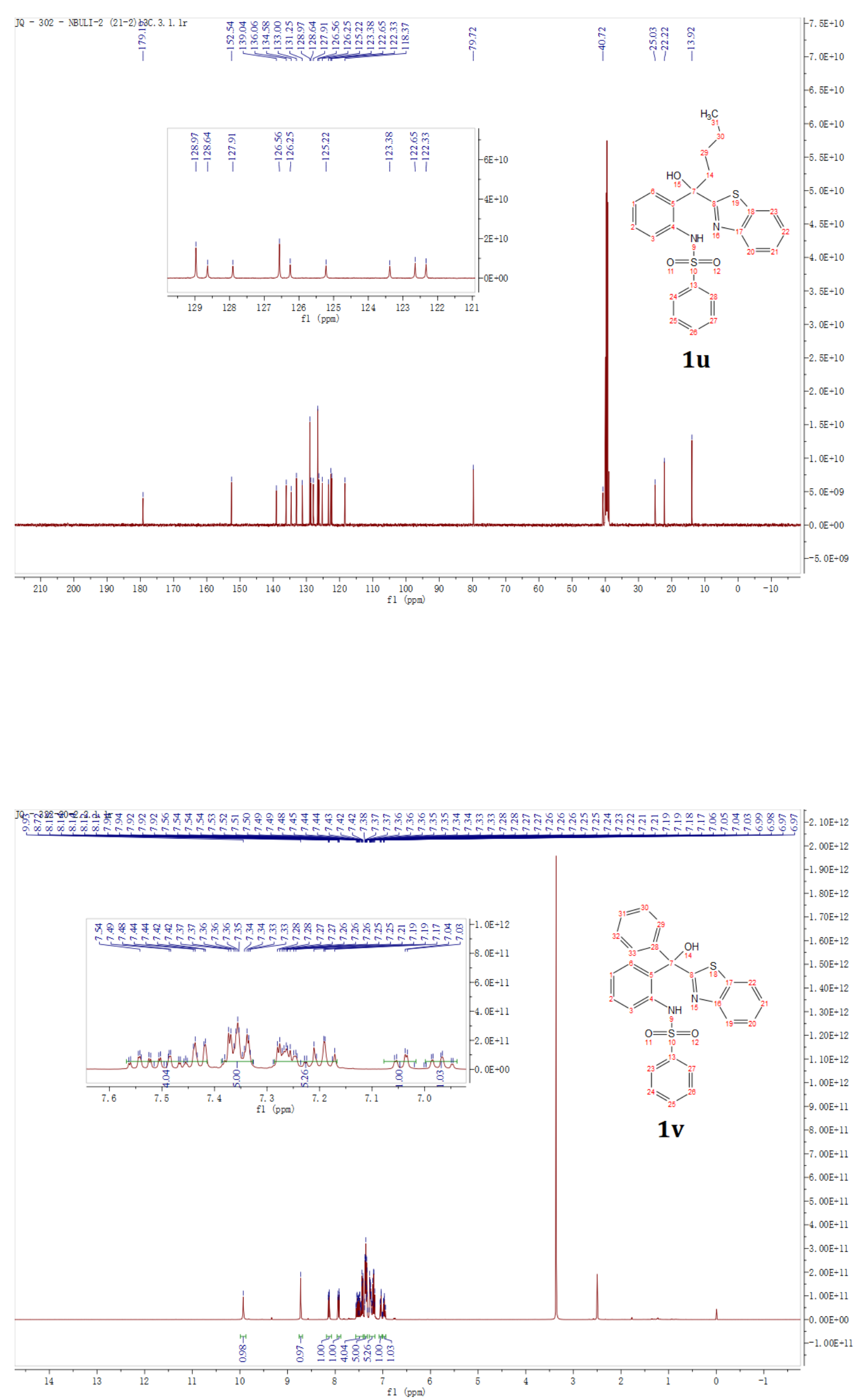

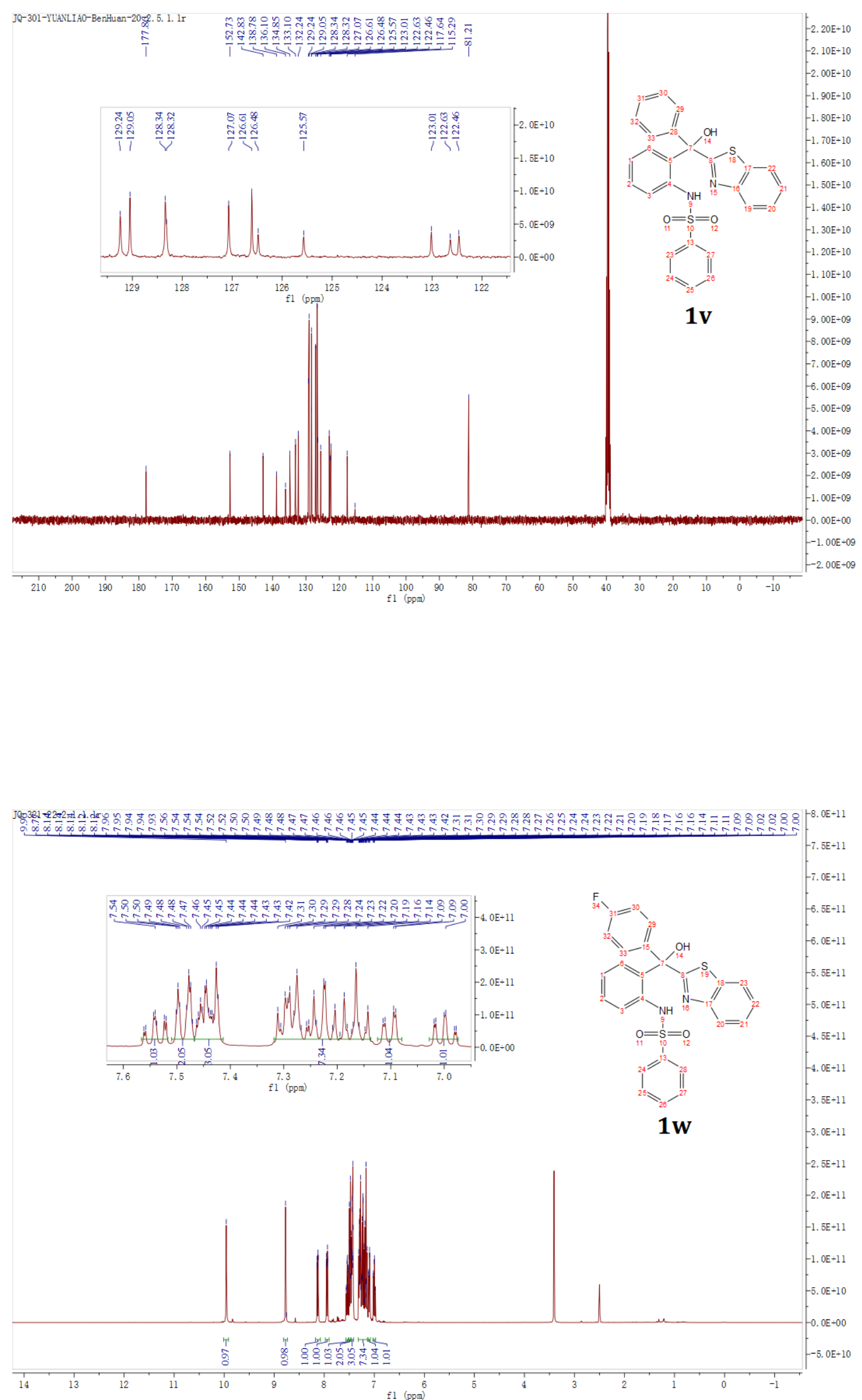

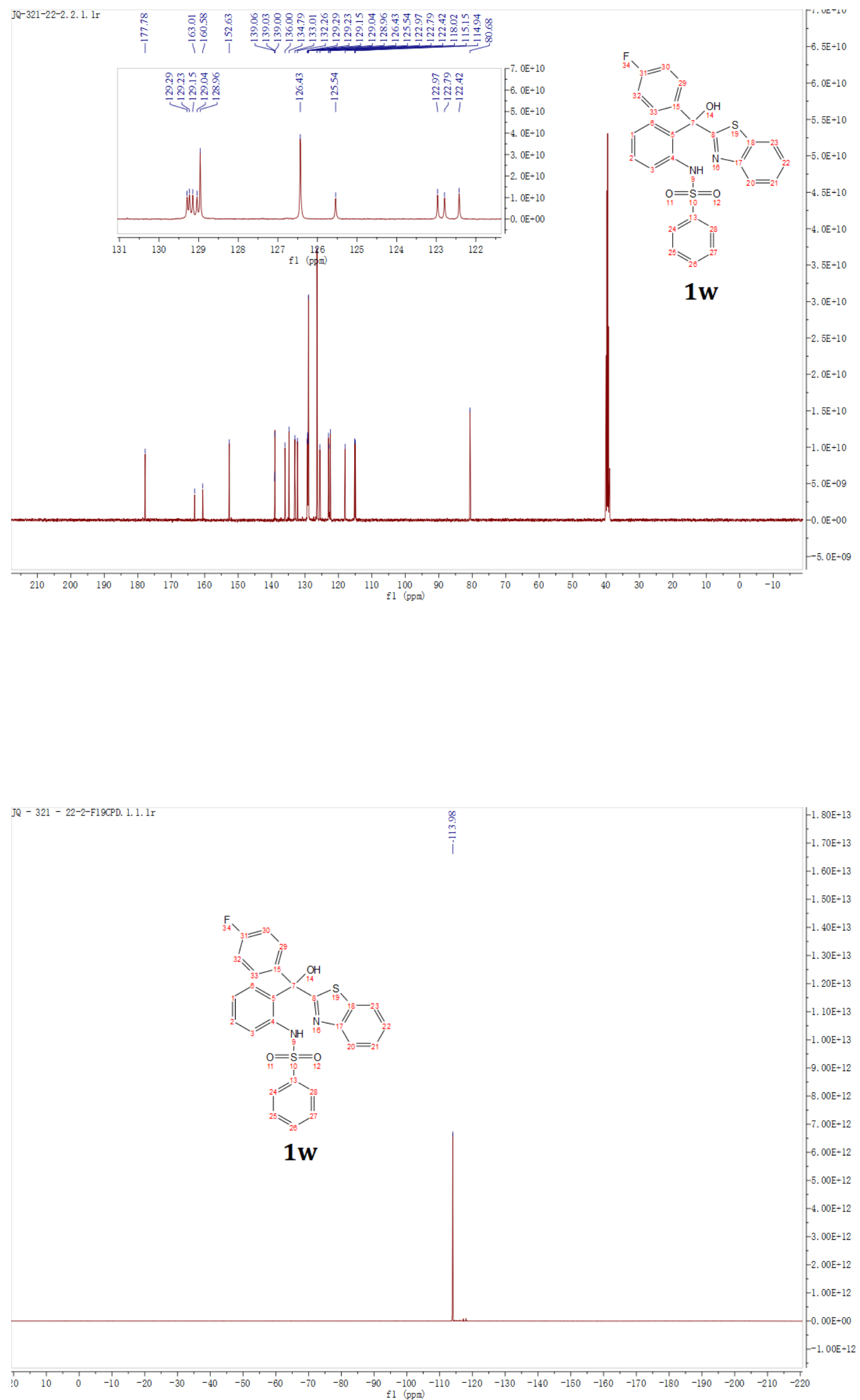

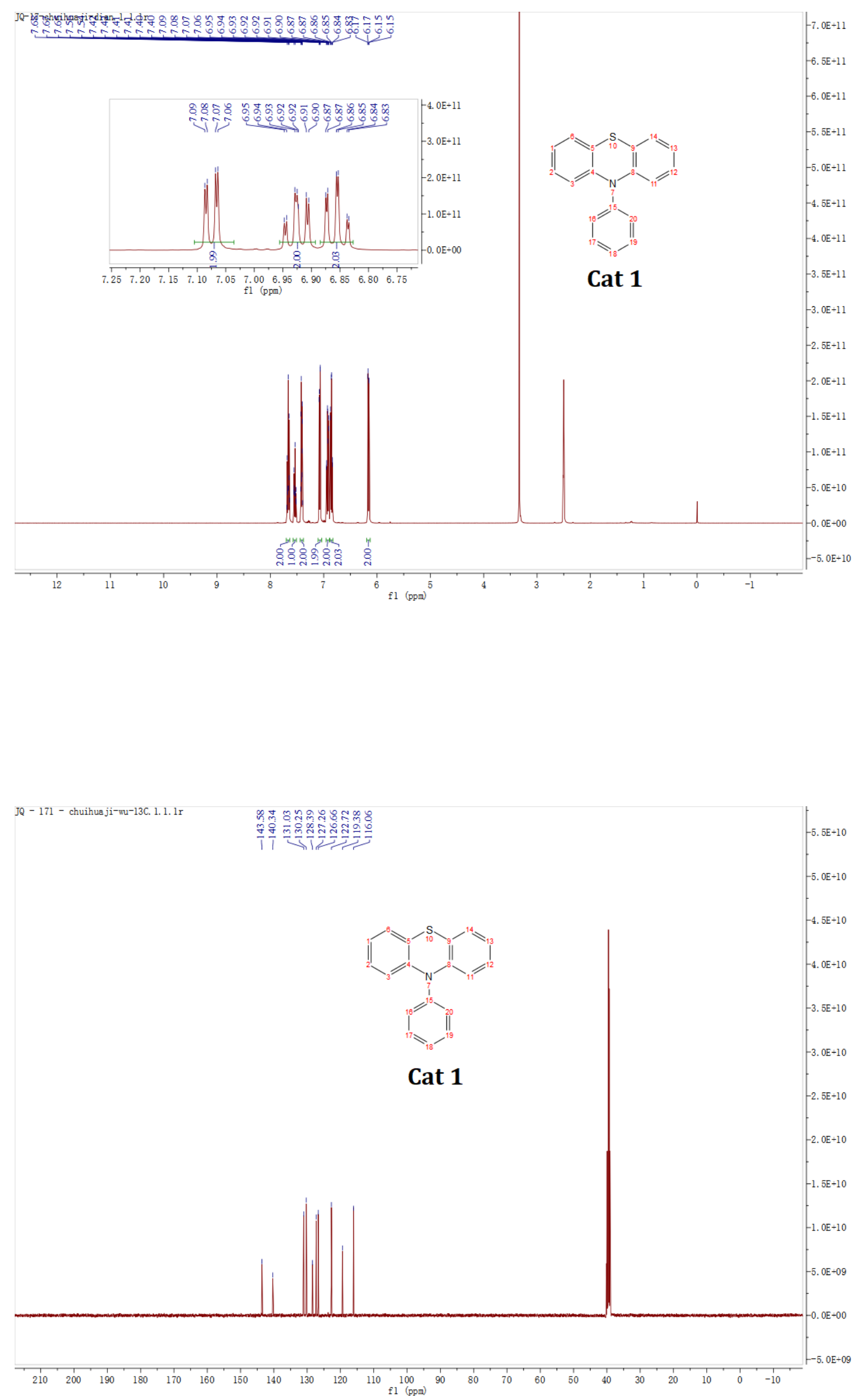

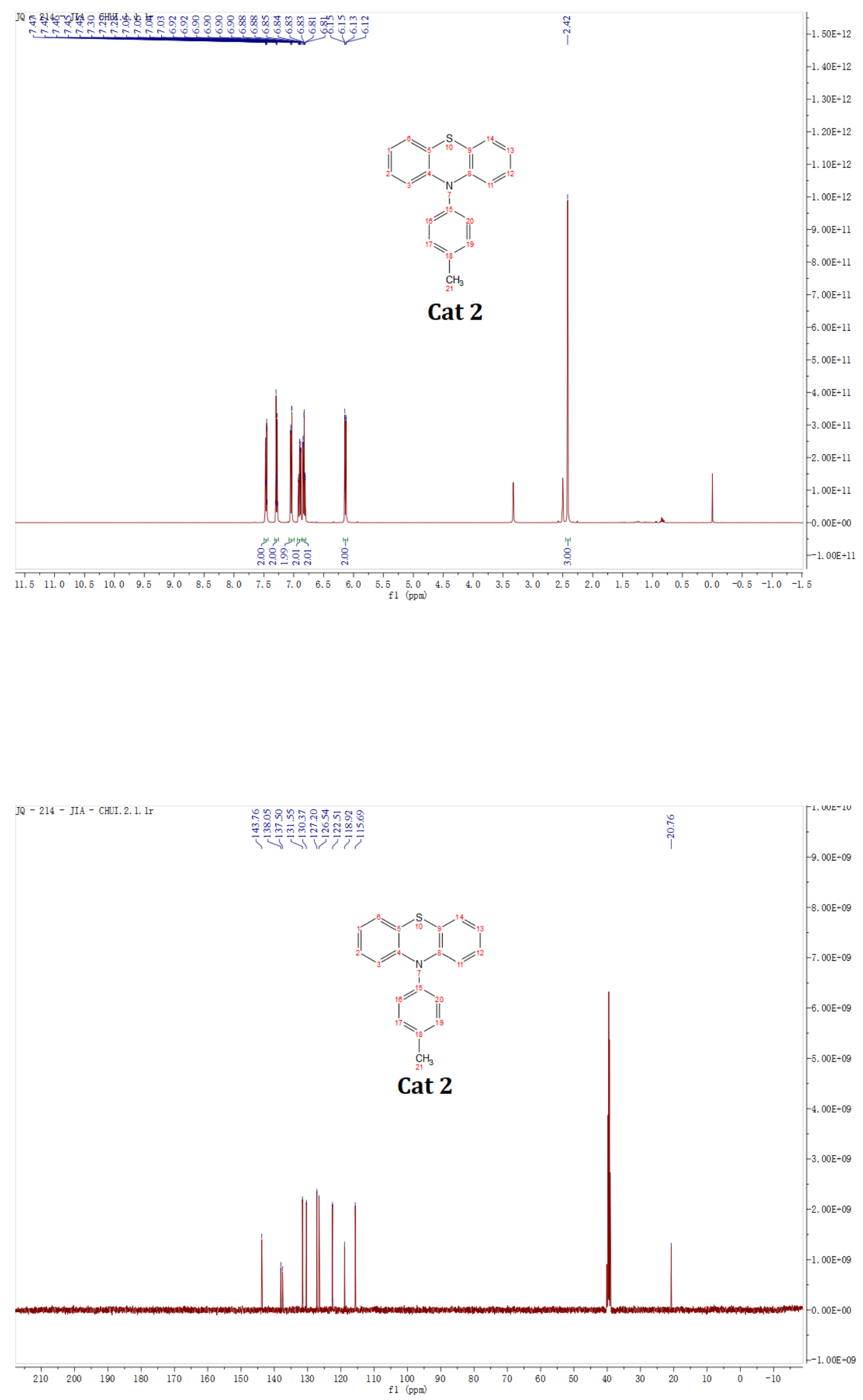

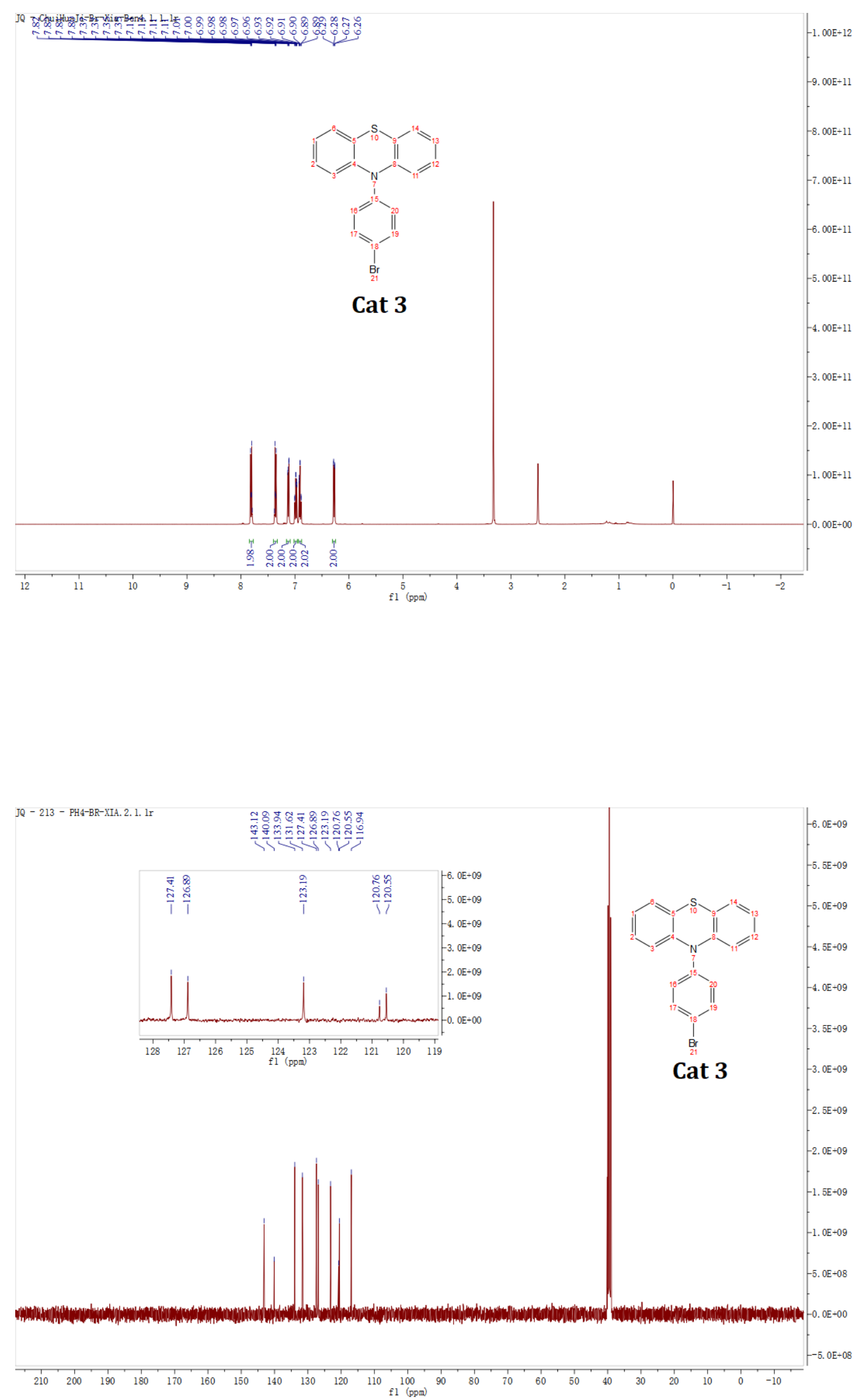

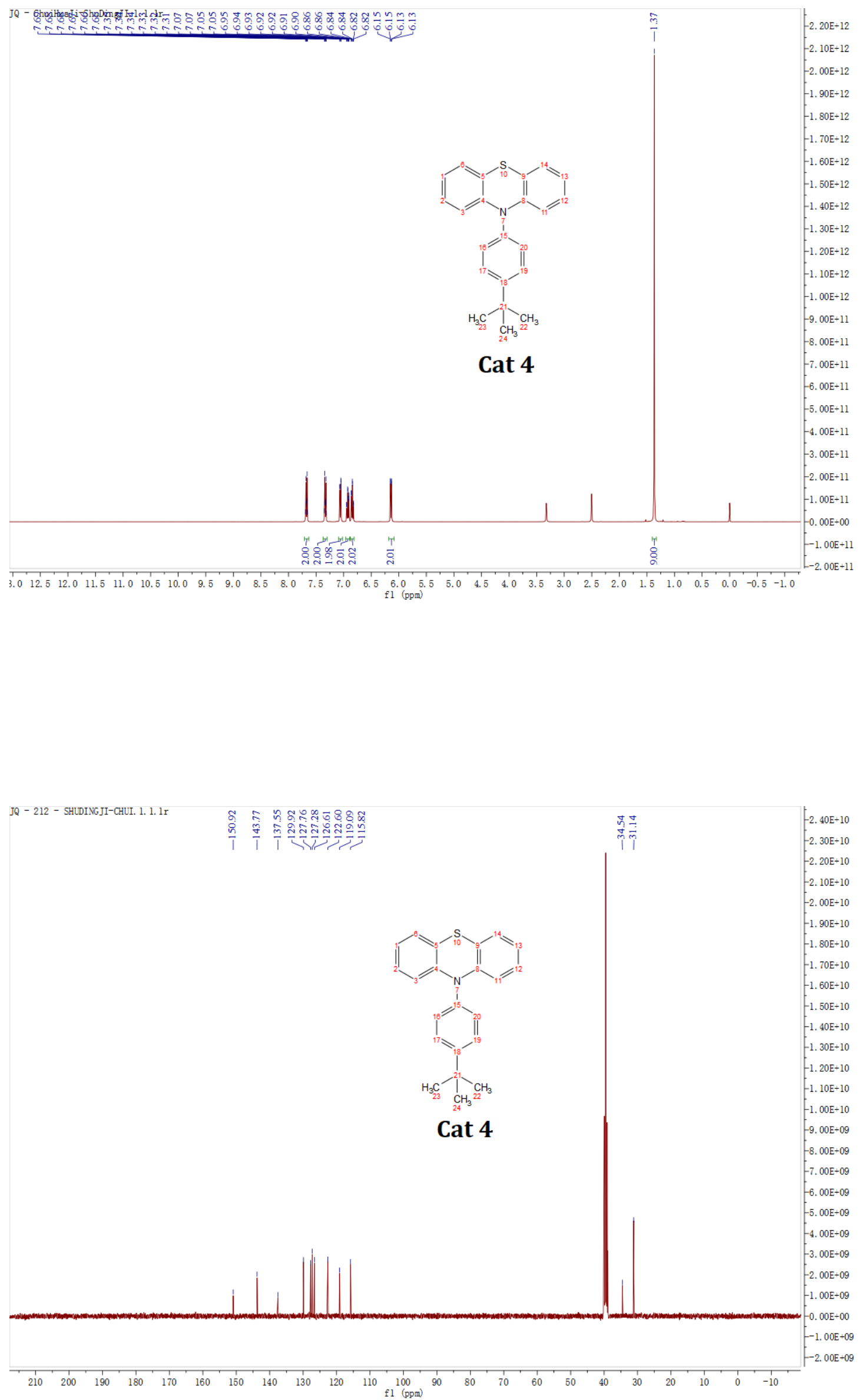

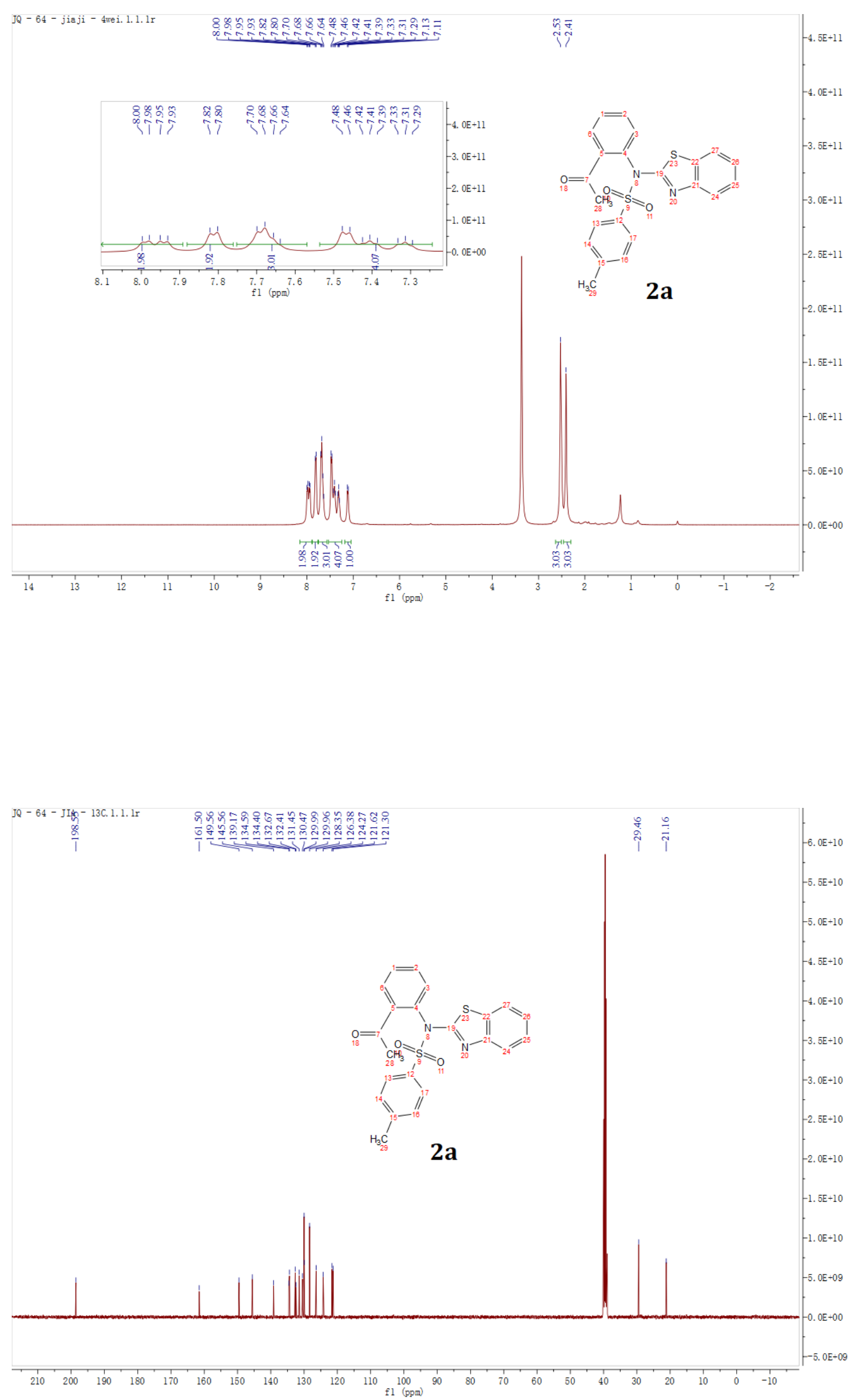

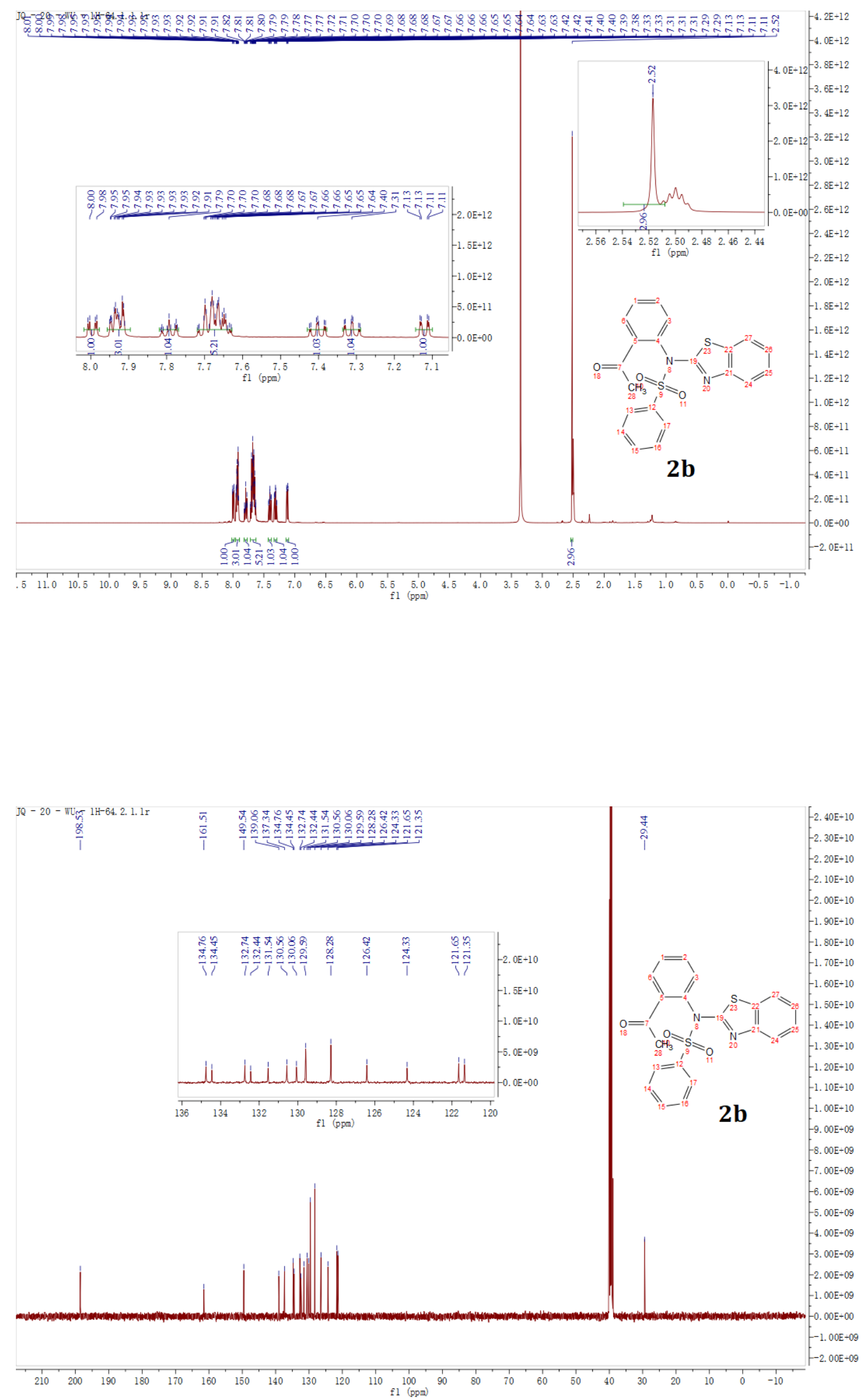

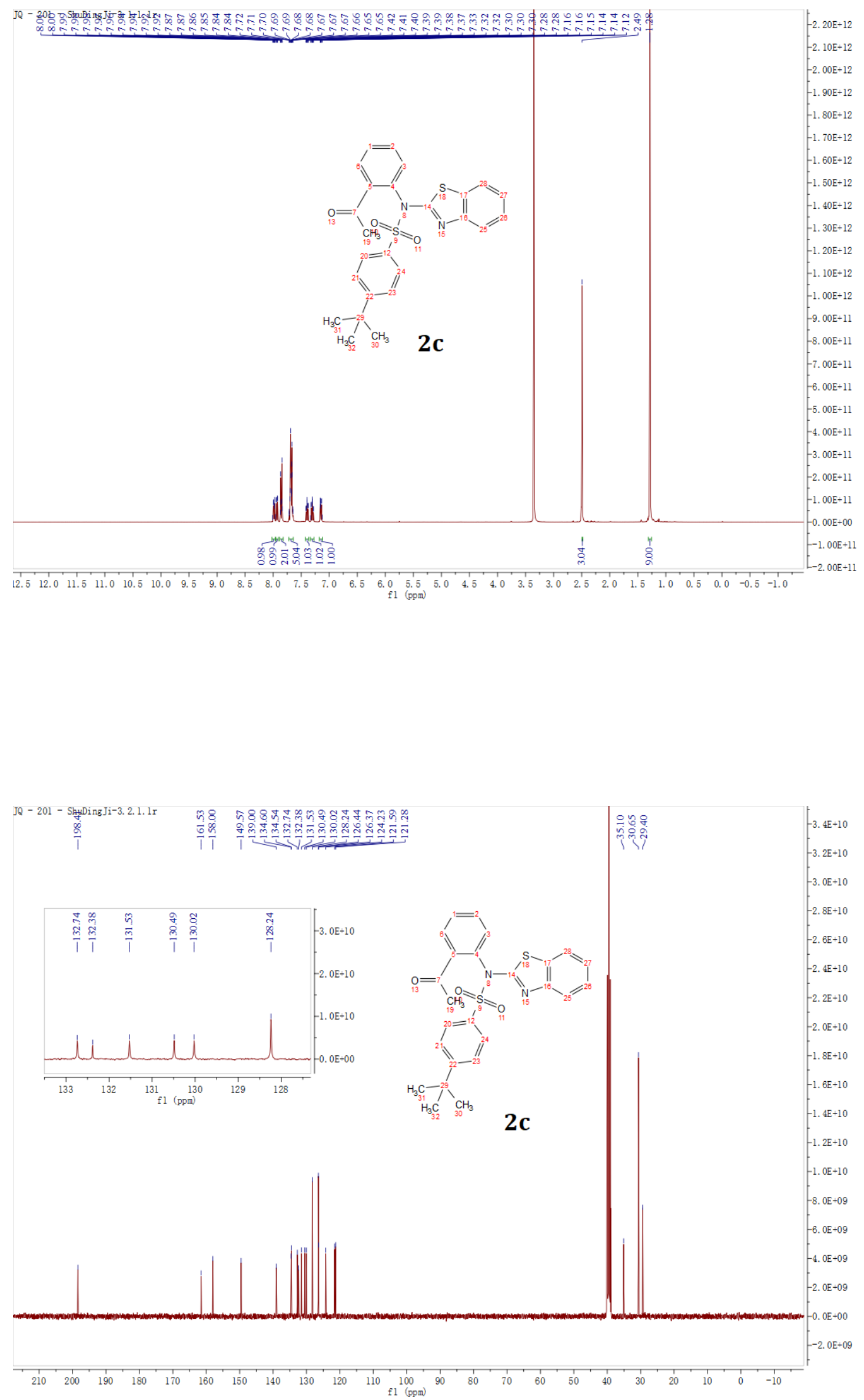


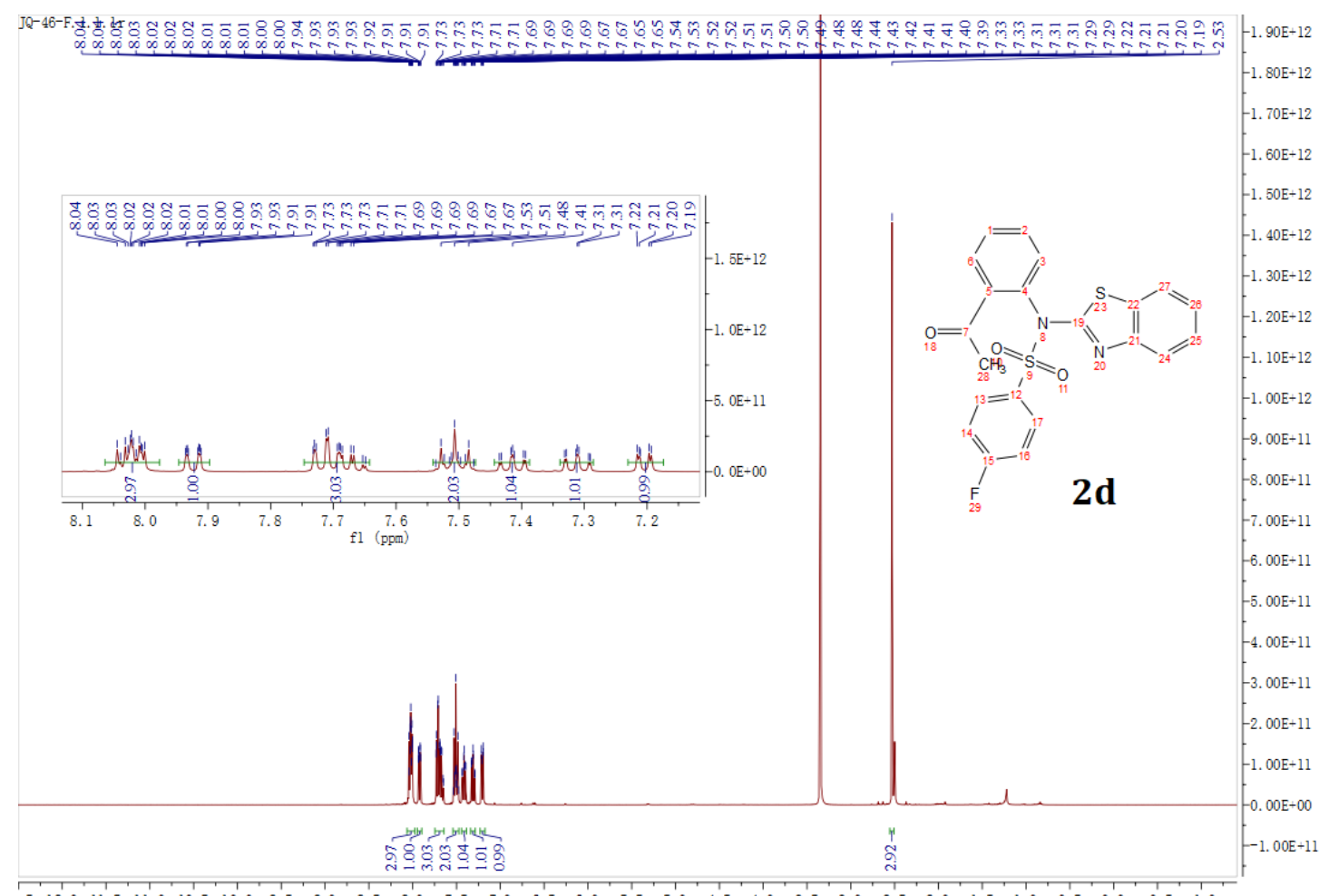

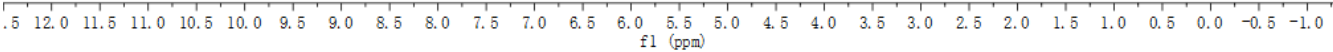

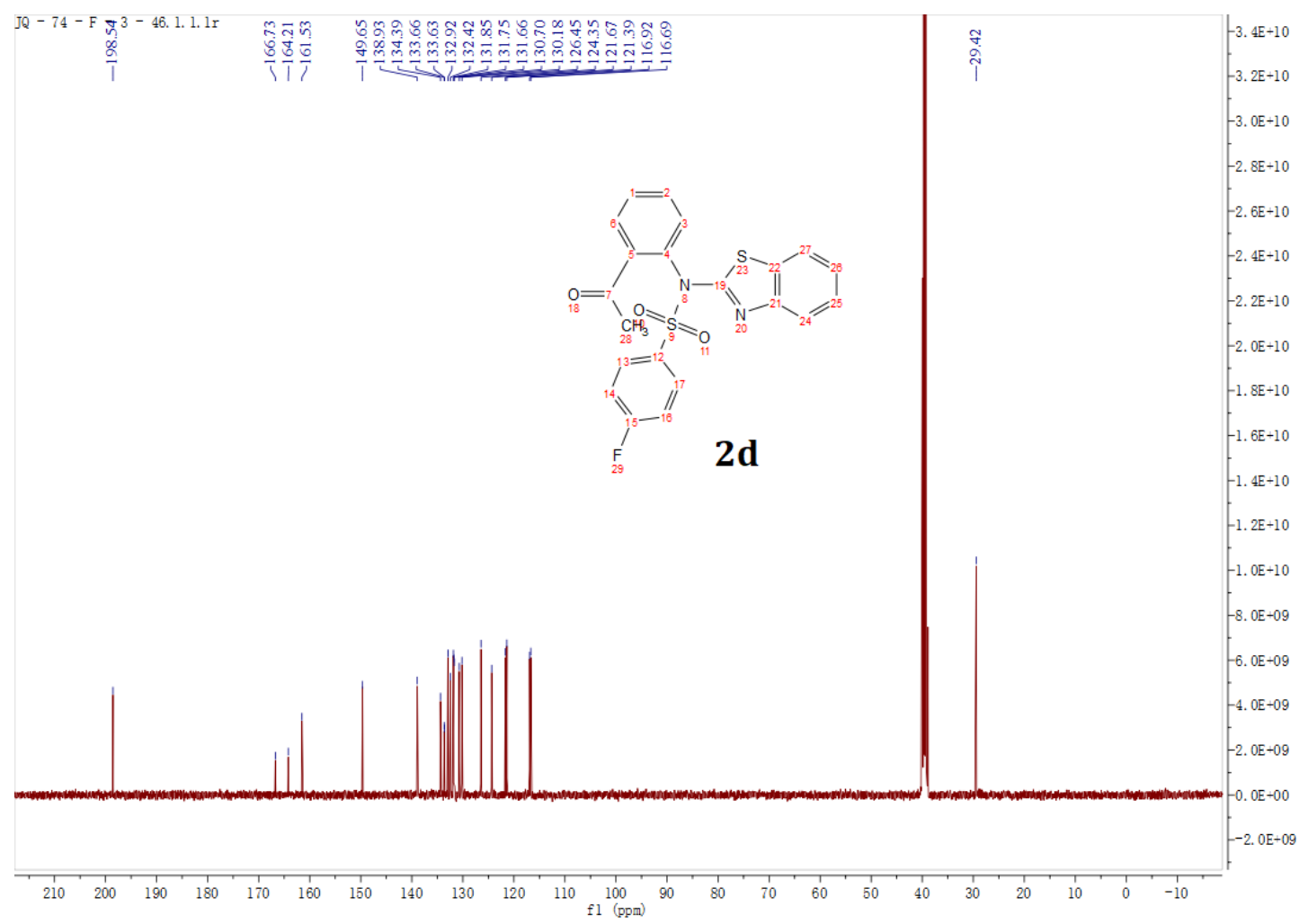



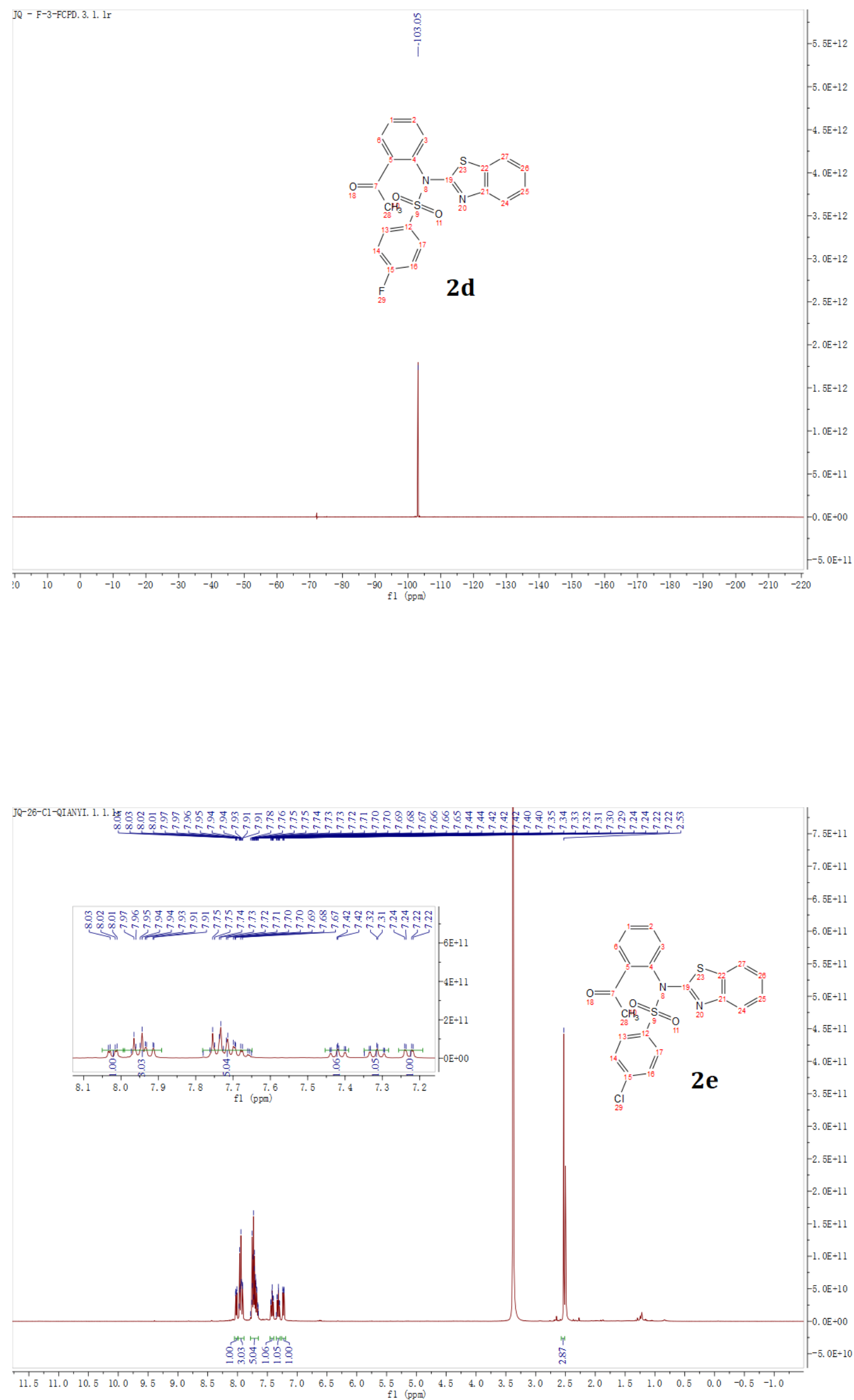

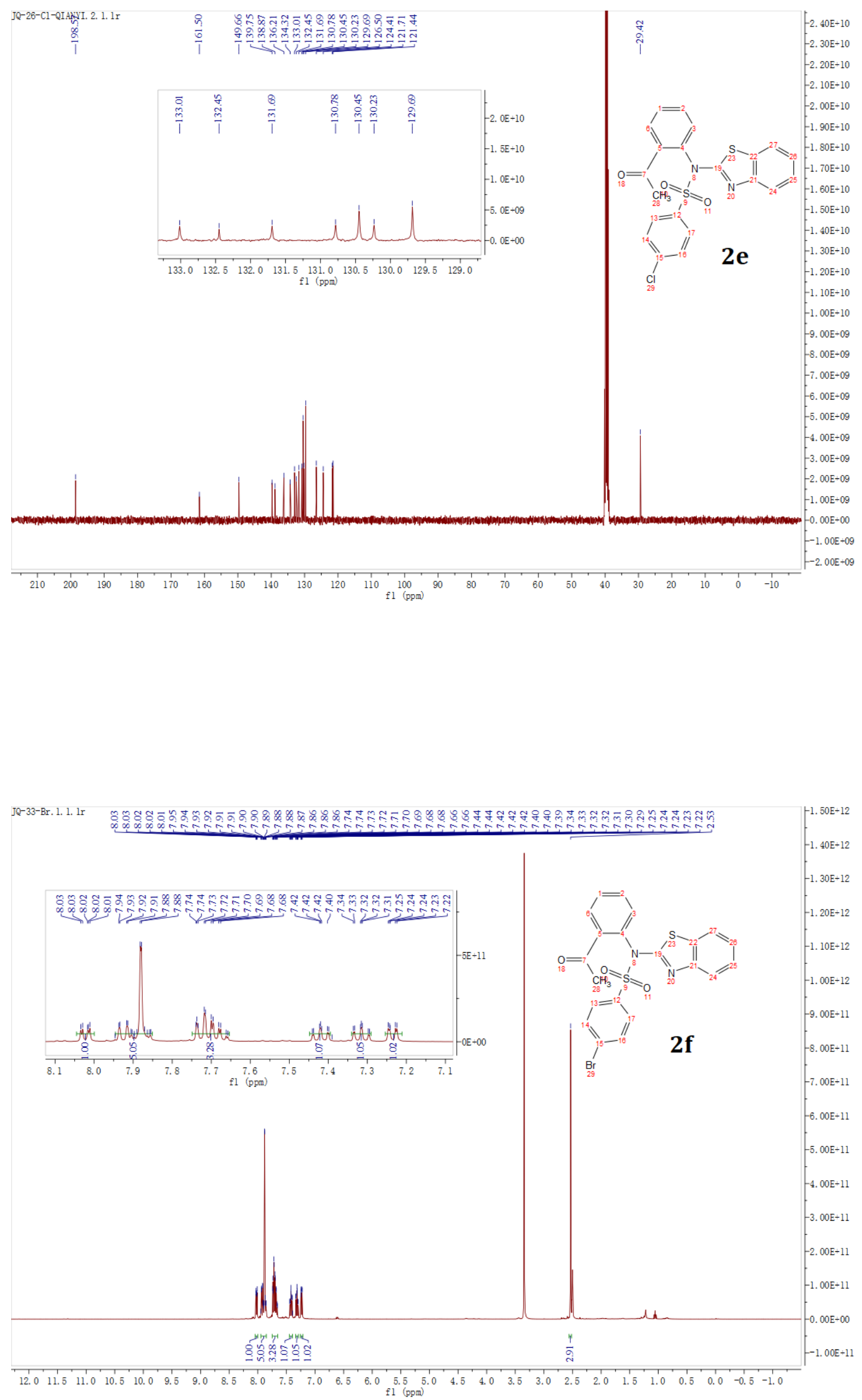

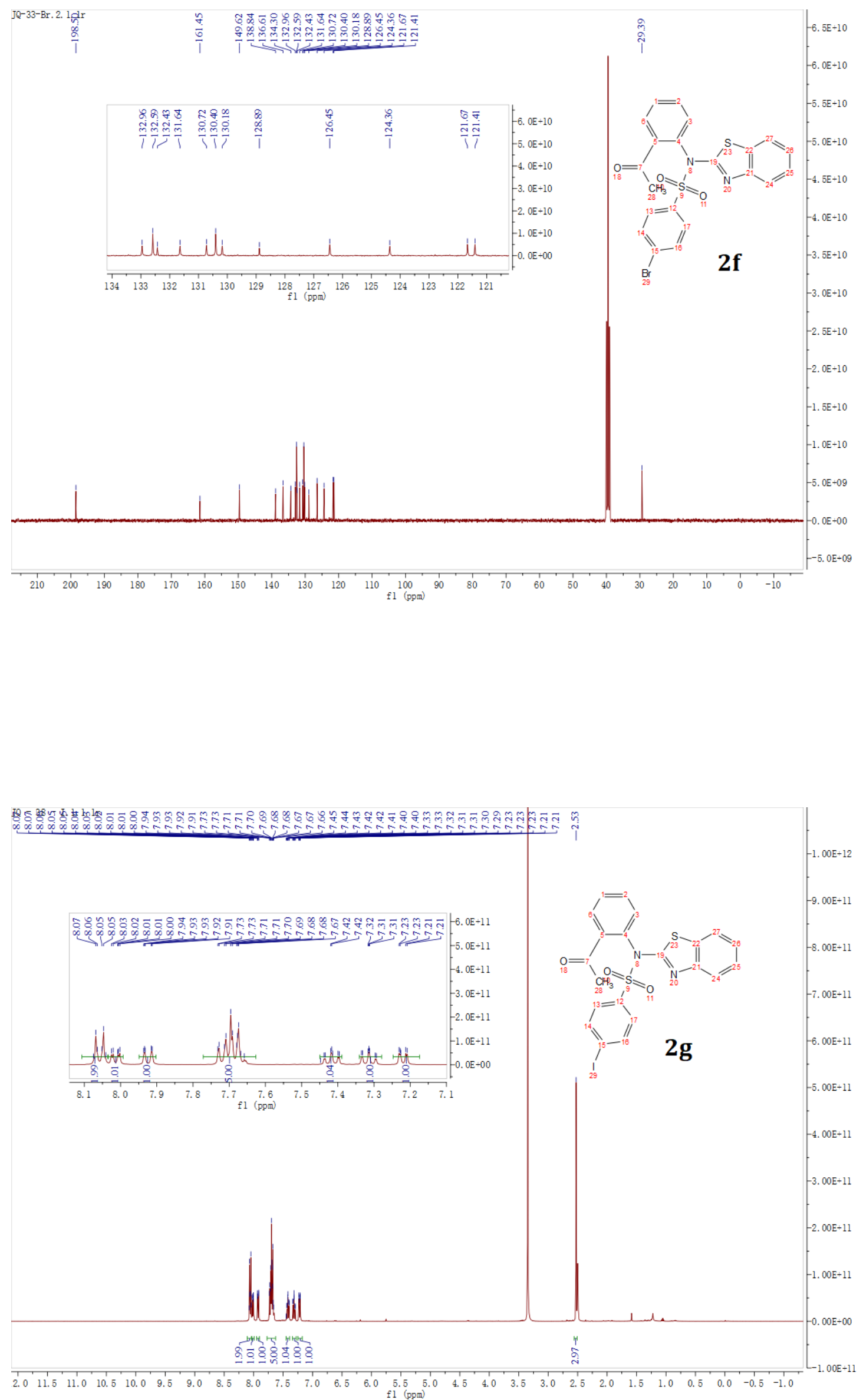

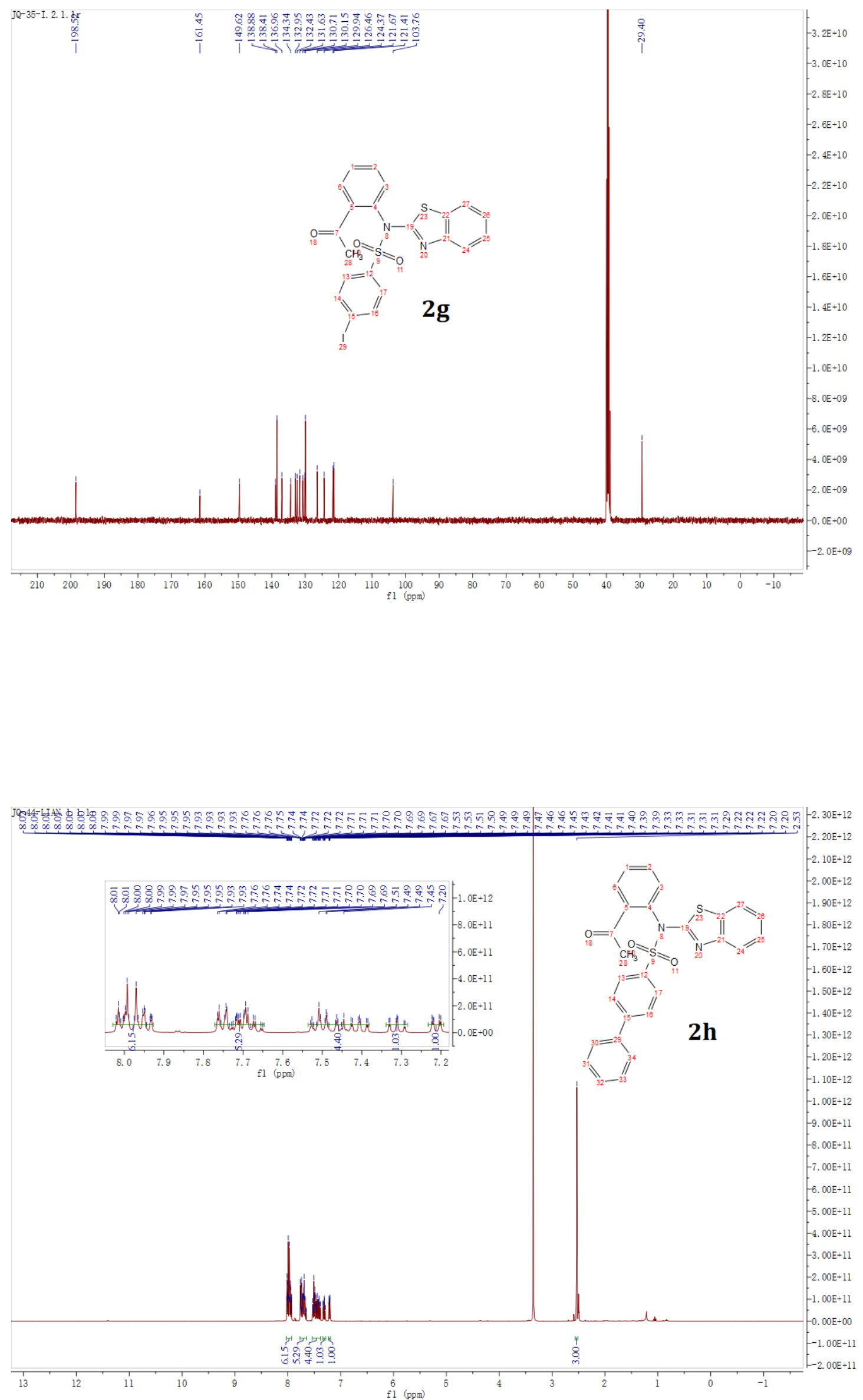

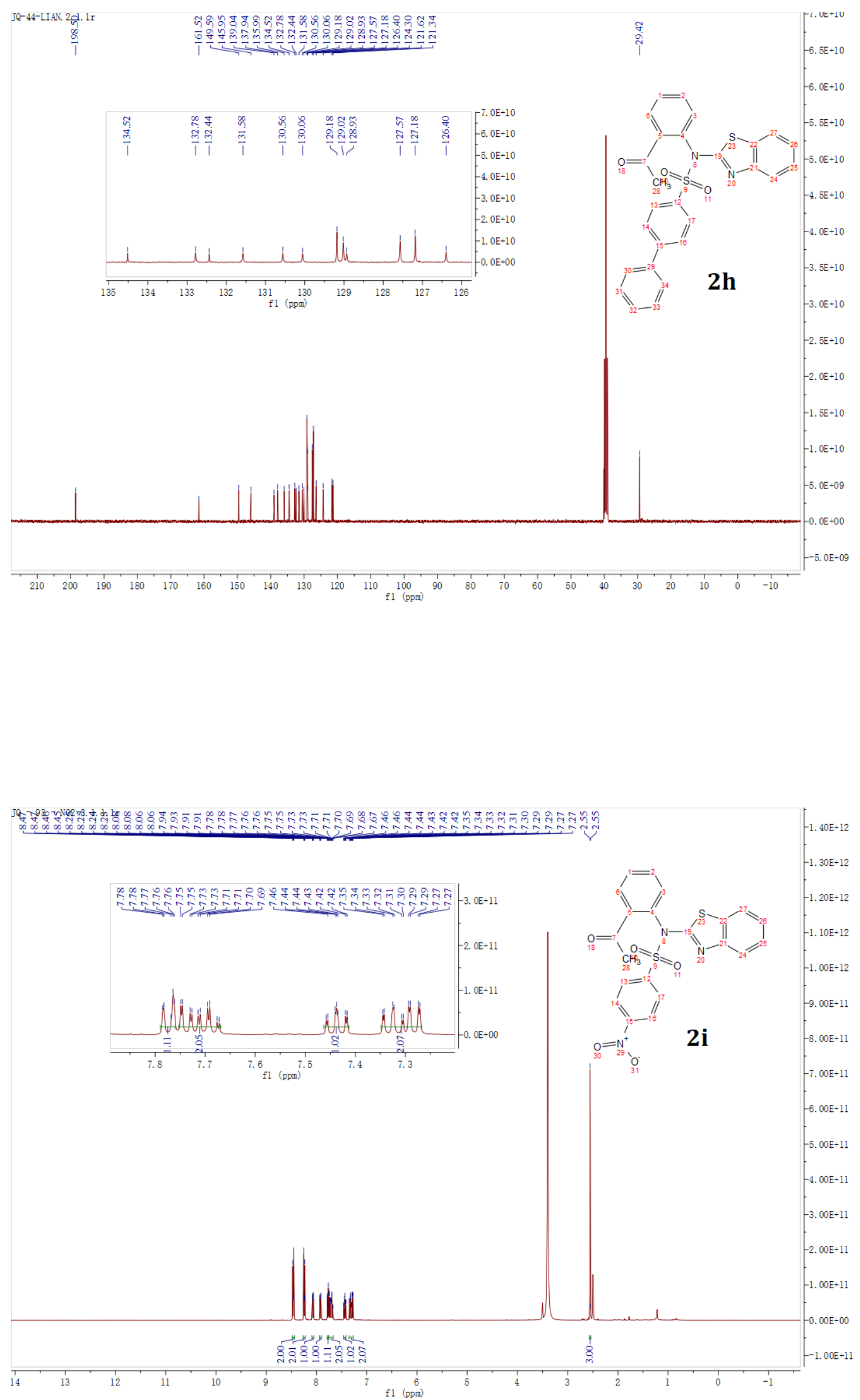

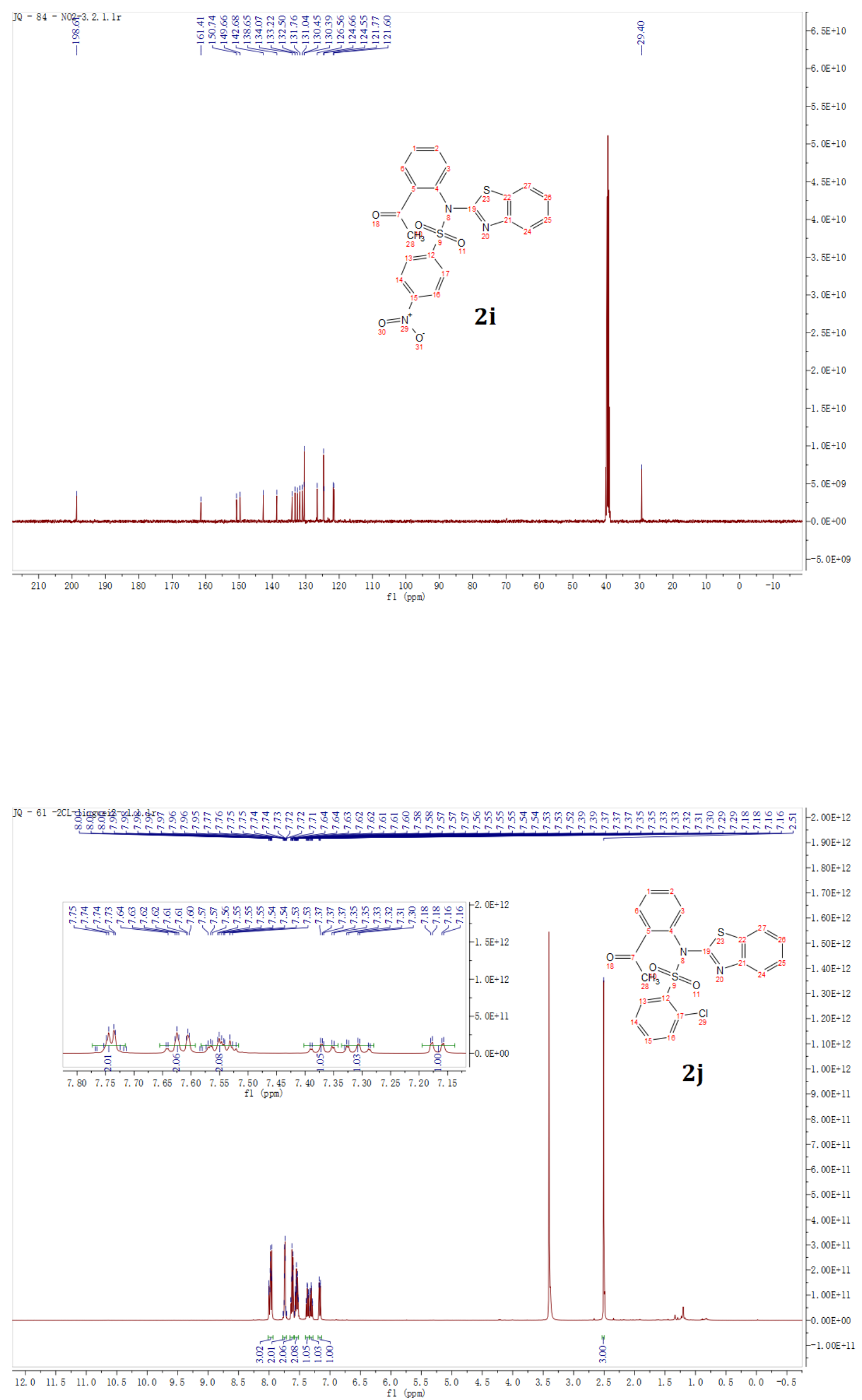

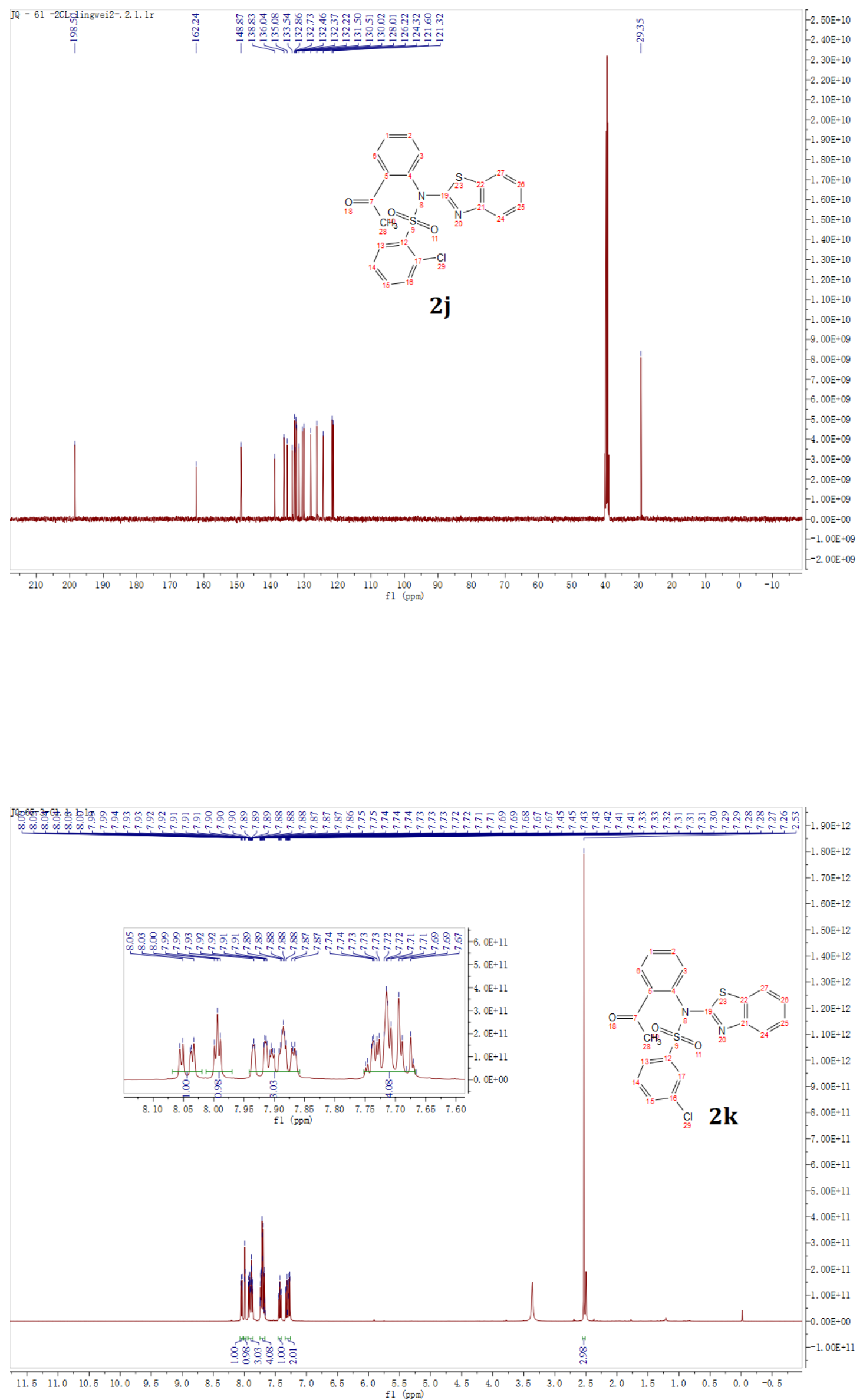

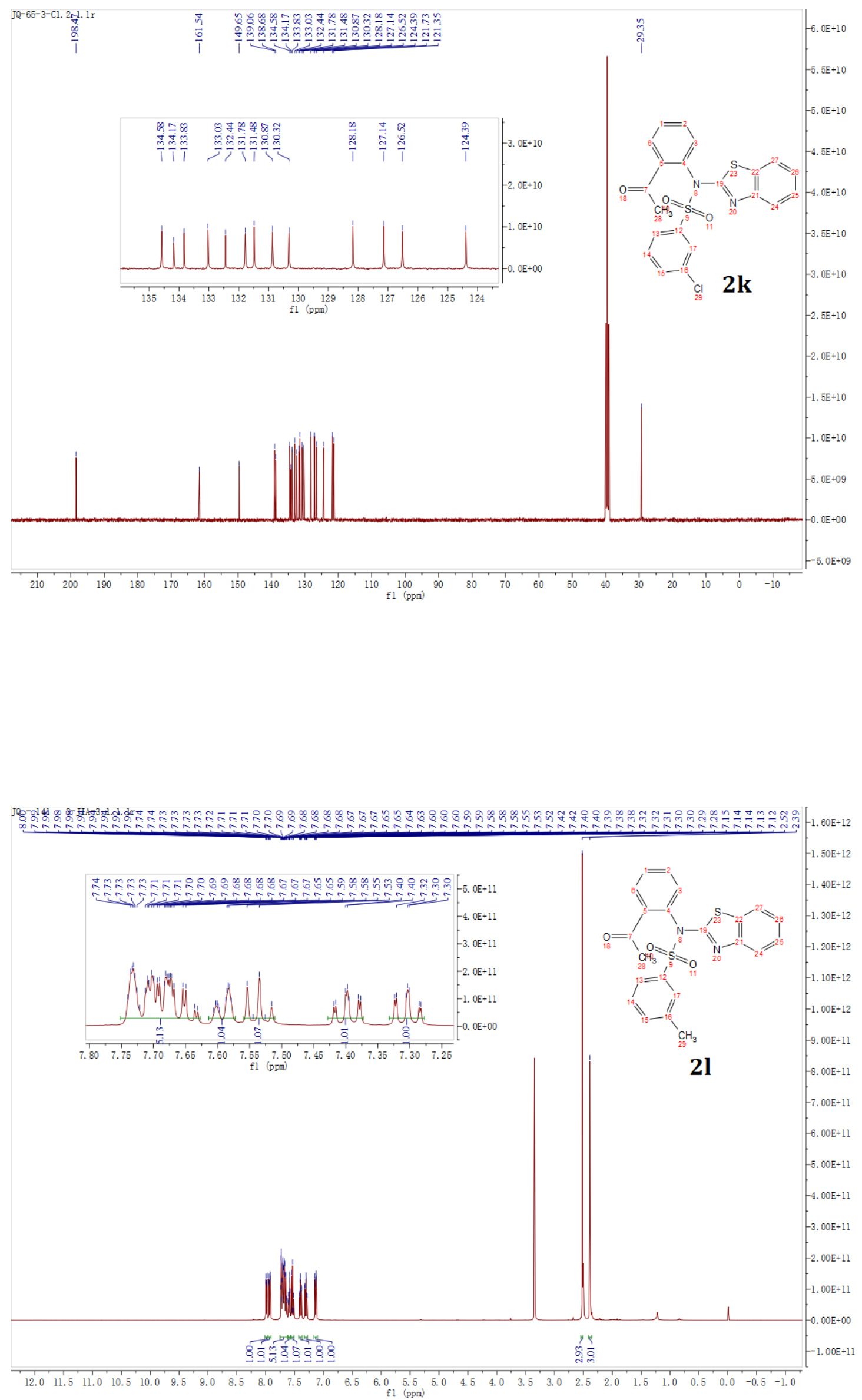

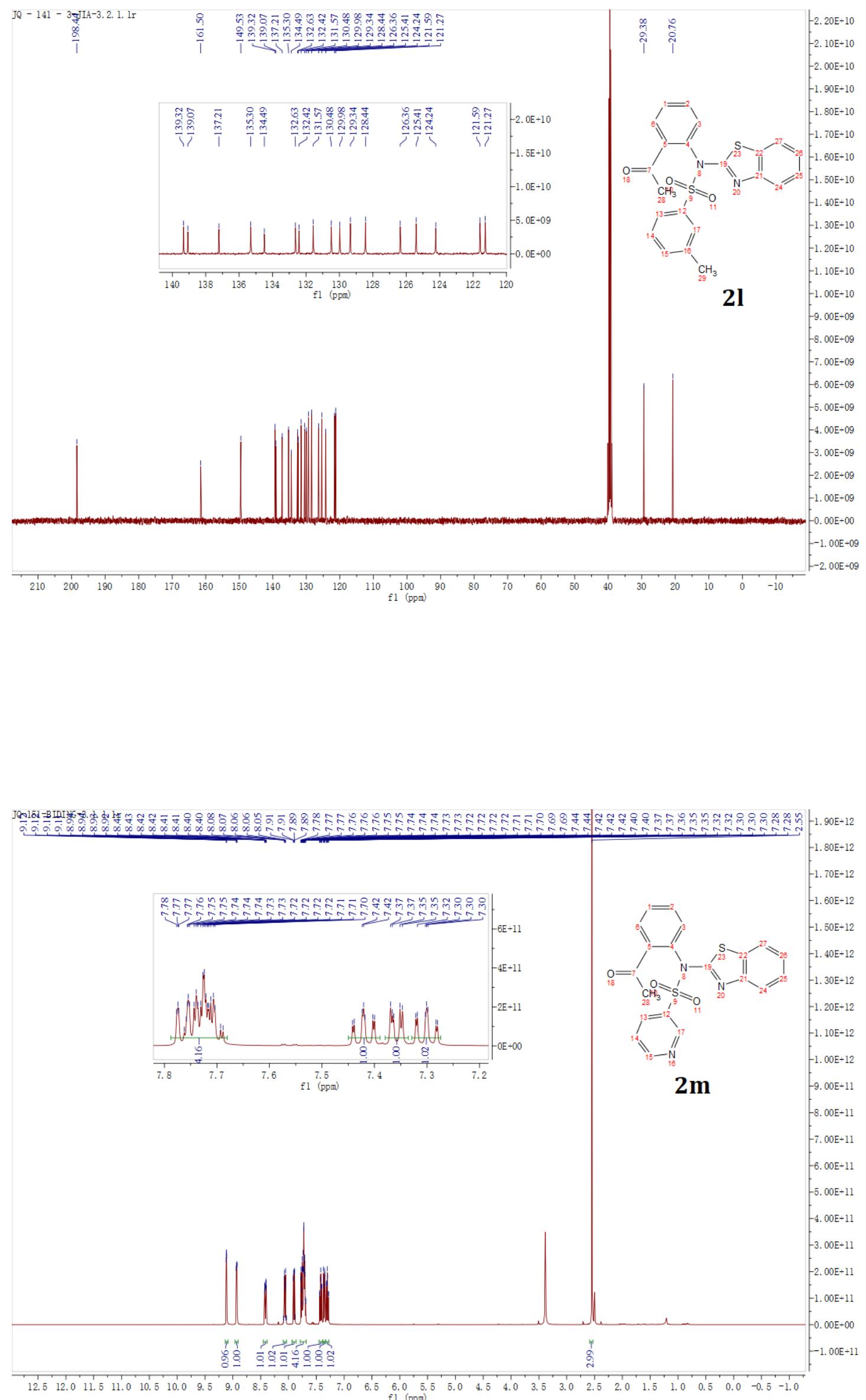

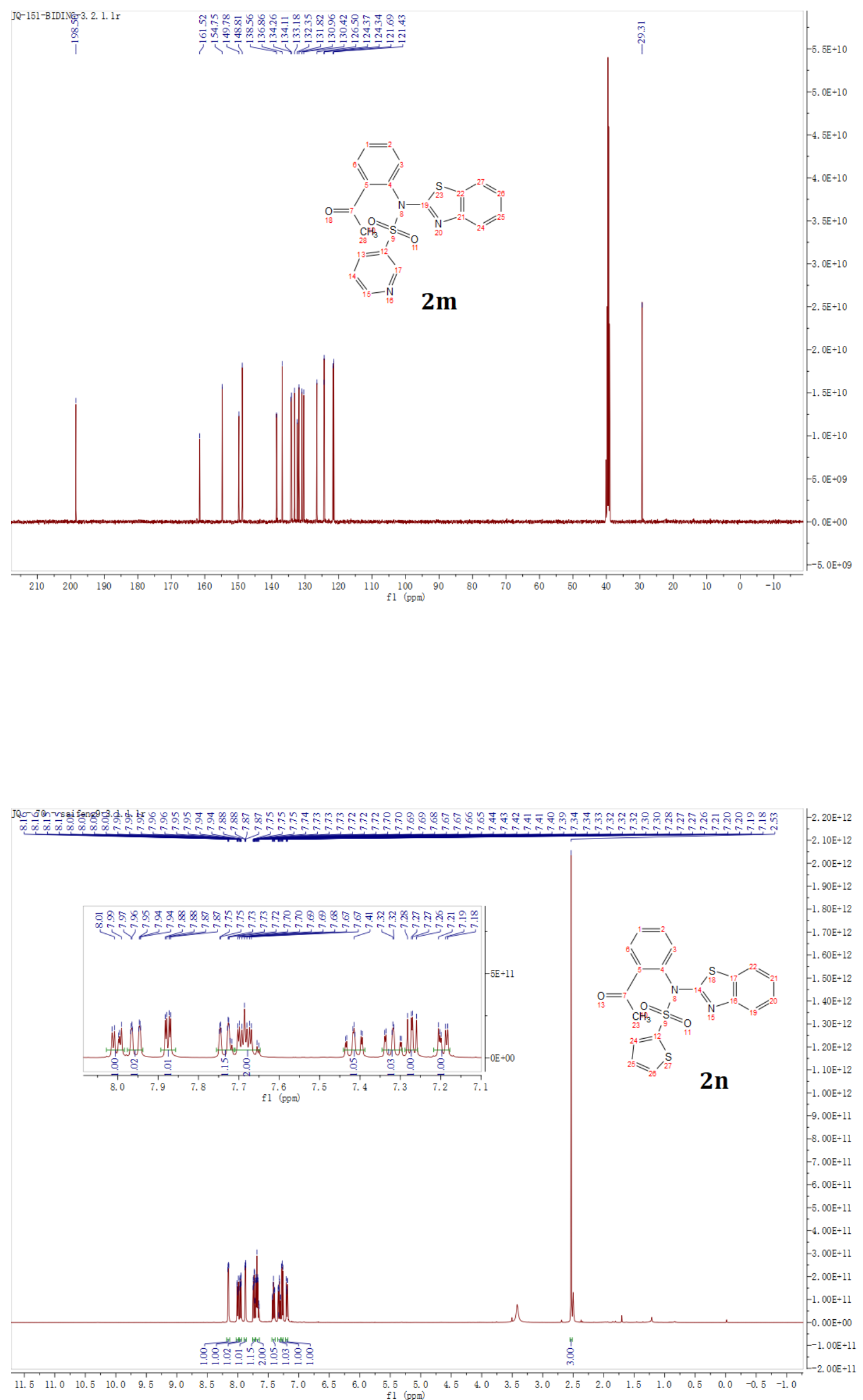

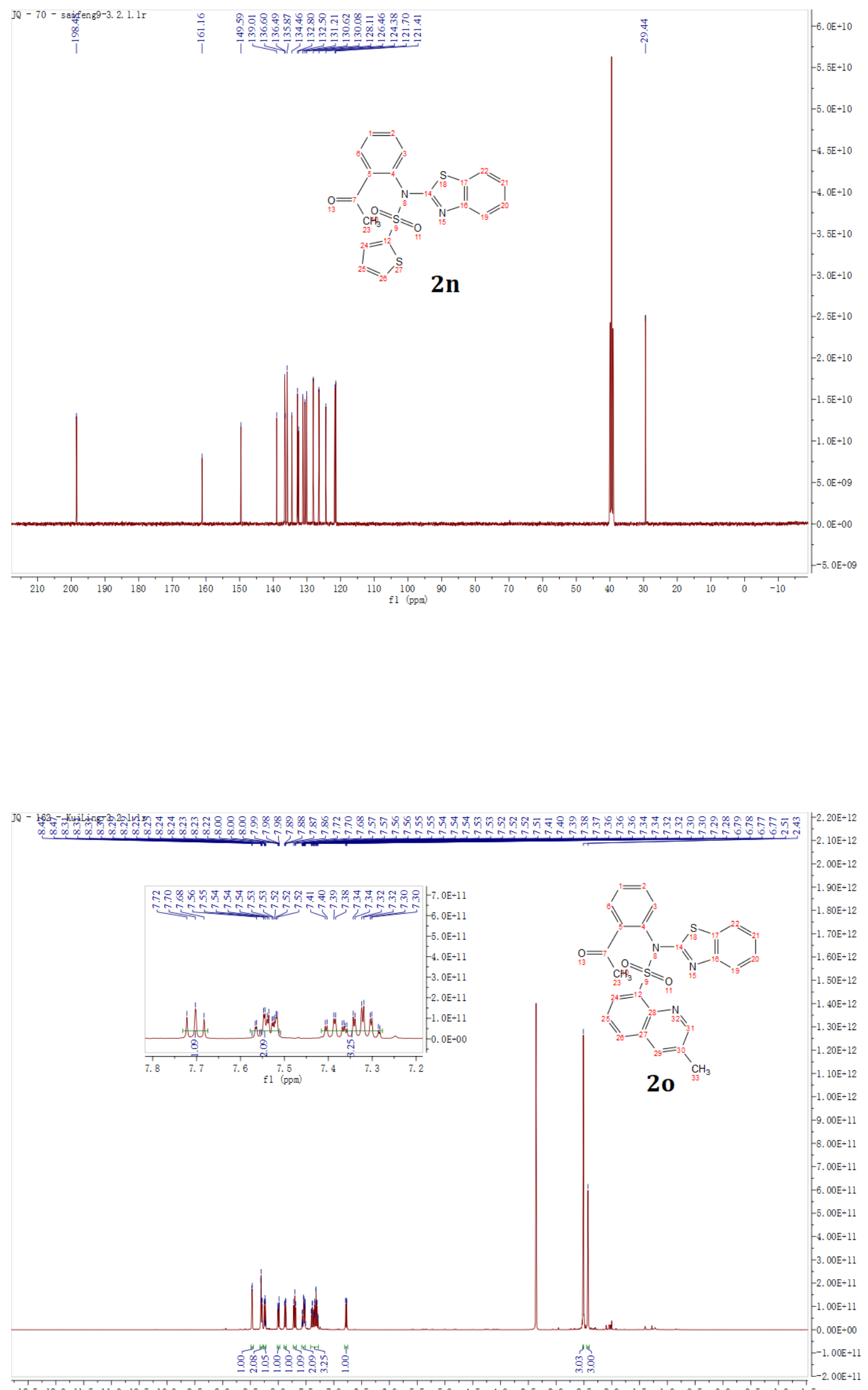

$\begin{array}{lllllllllllllllllllllllllllllllllllllllll}12.5 & 12.0 & 11.5 & 11.0 & 10.5 & 10.0 & 9.5 & 9.0 & 8.5 & 8.0 & 7.5 & 7.0 & 6.5 & 6.0 & 5.5 & 5.0 & 4.5 & 4.0 & 3.5 & 3.0 & 2.5 & 2.0 & 1.5 & 1.0 & 0.5 & 0.0 & -0.5 & -1.0 & -1.5\end{array}$ 

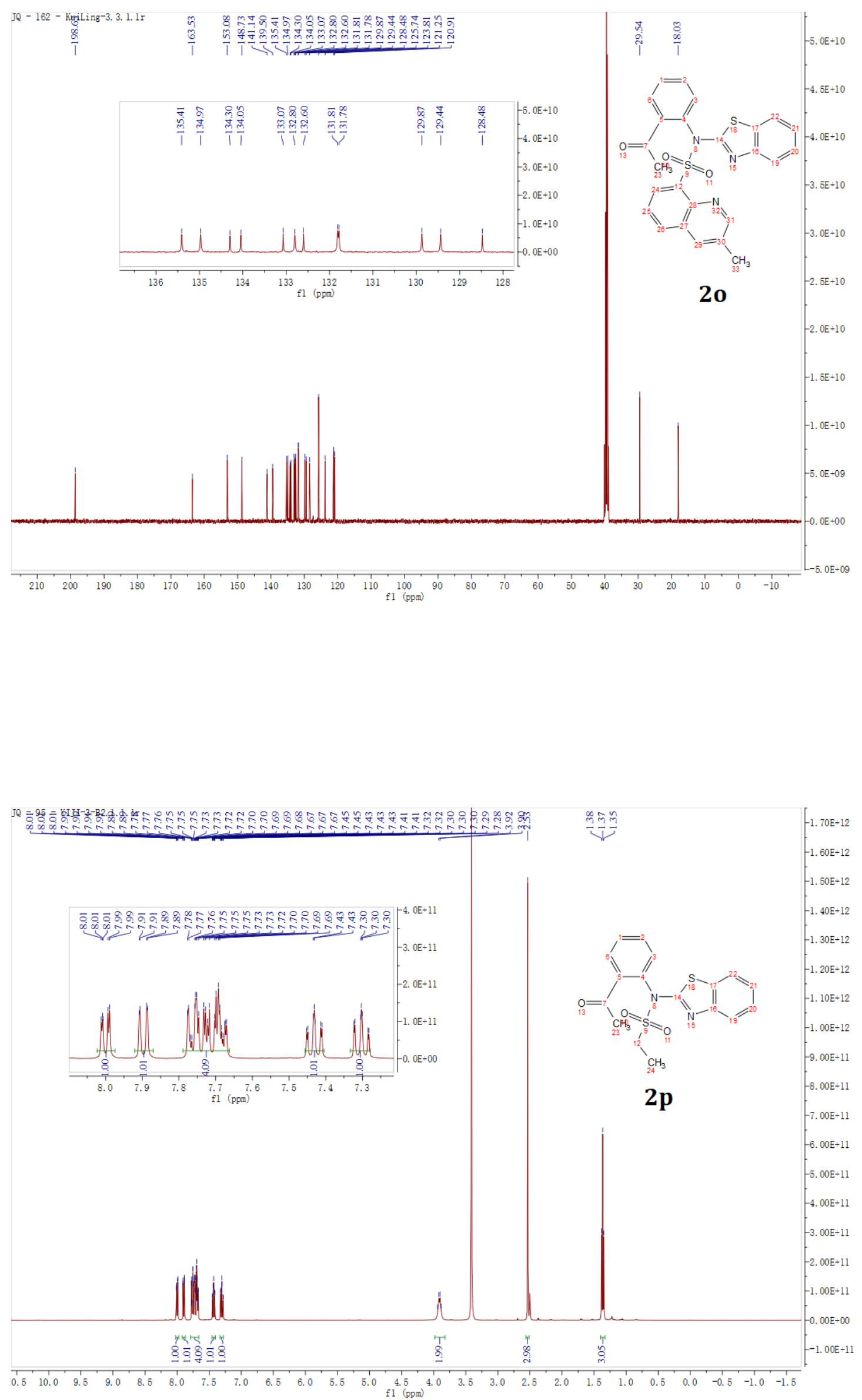

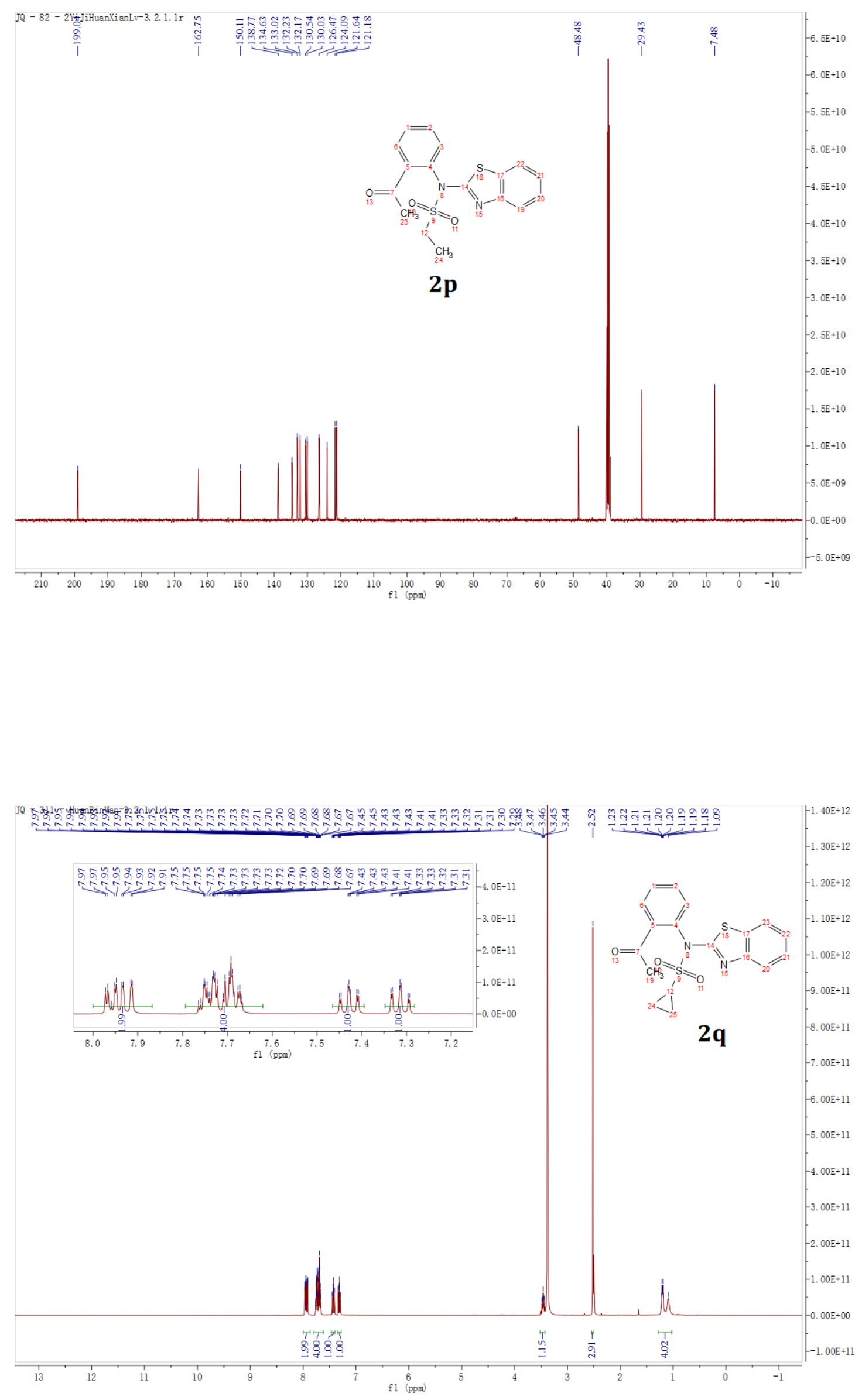

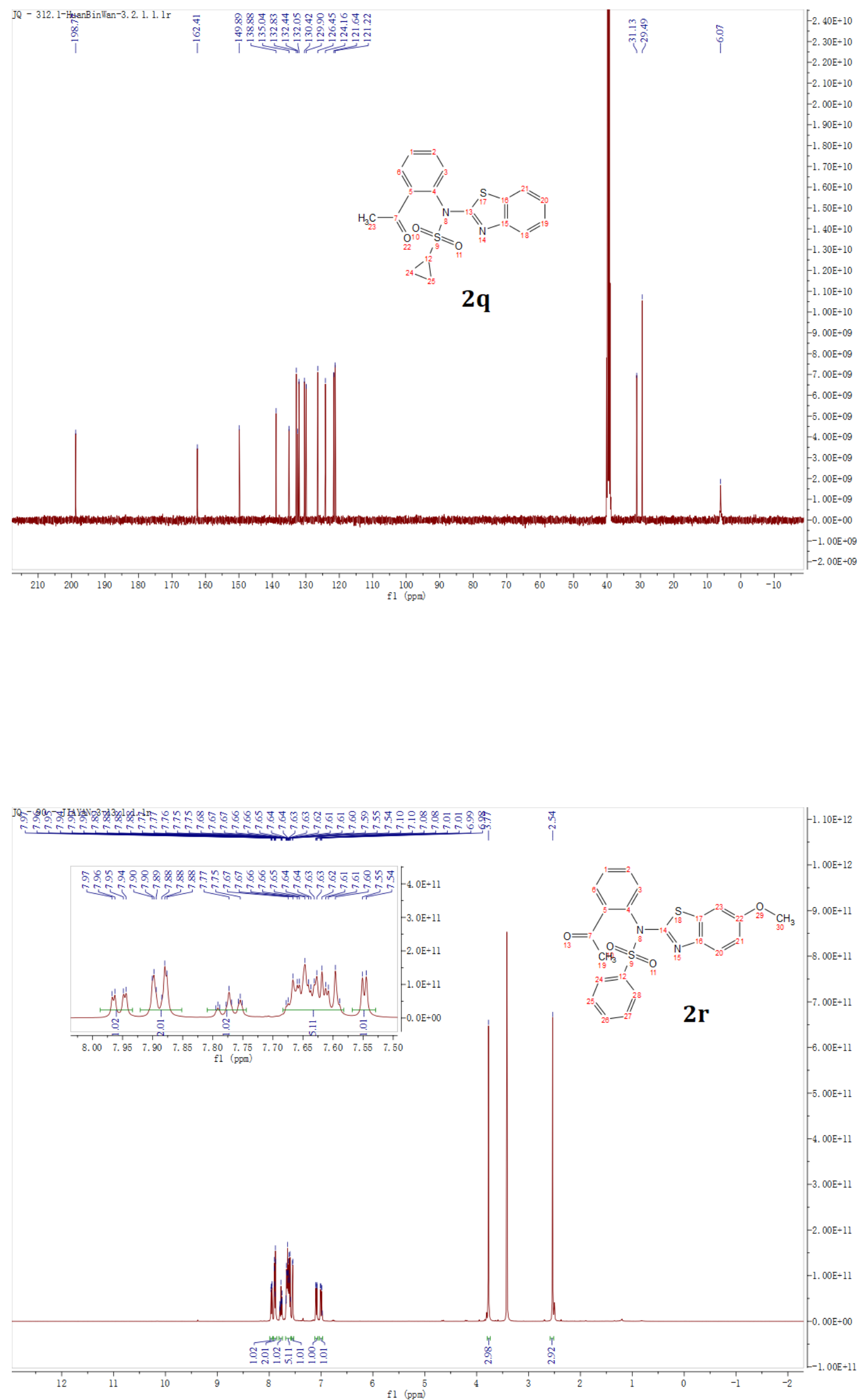

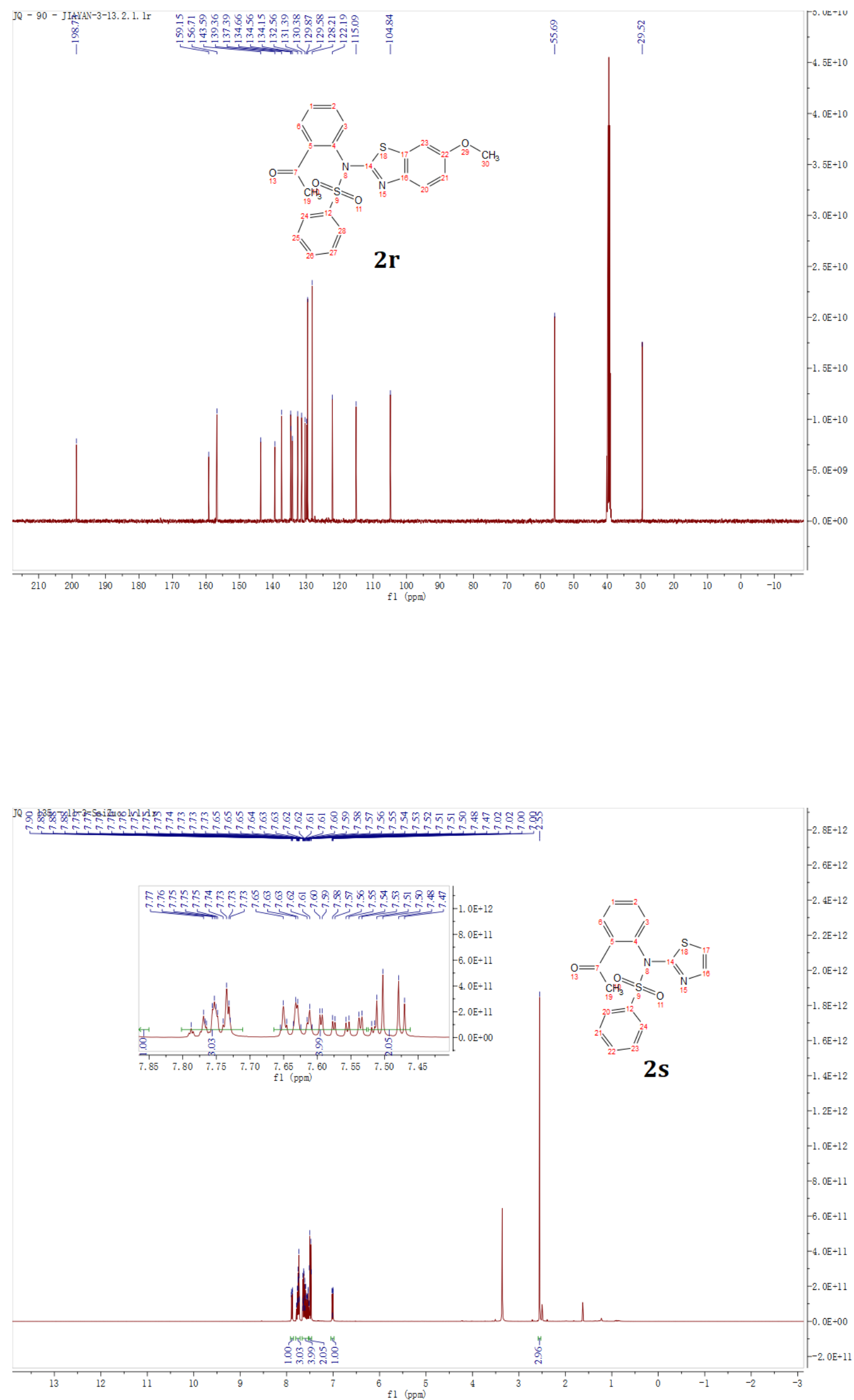

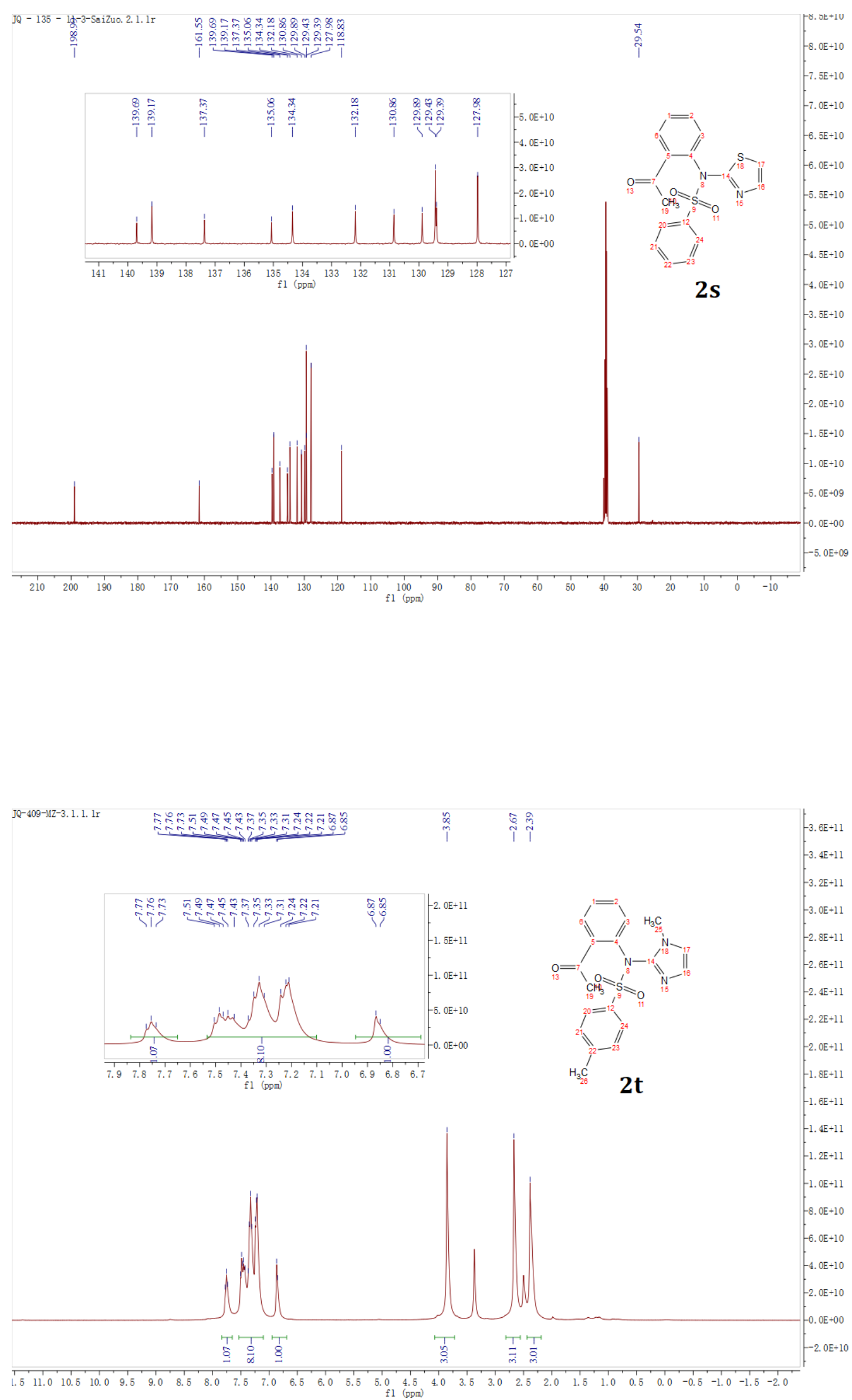

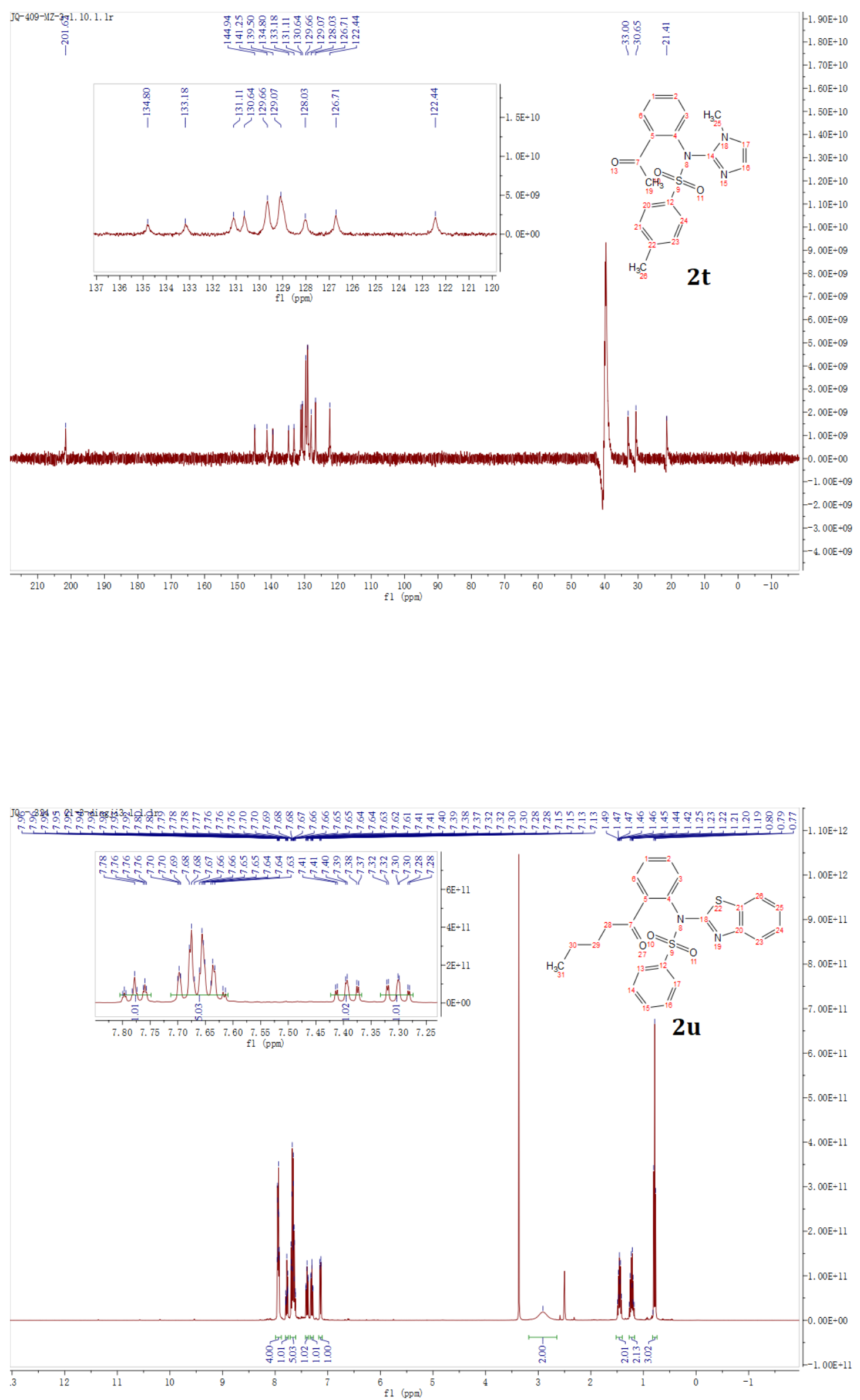

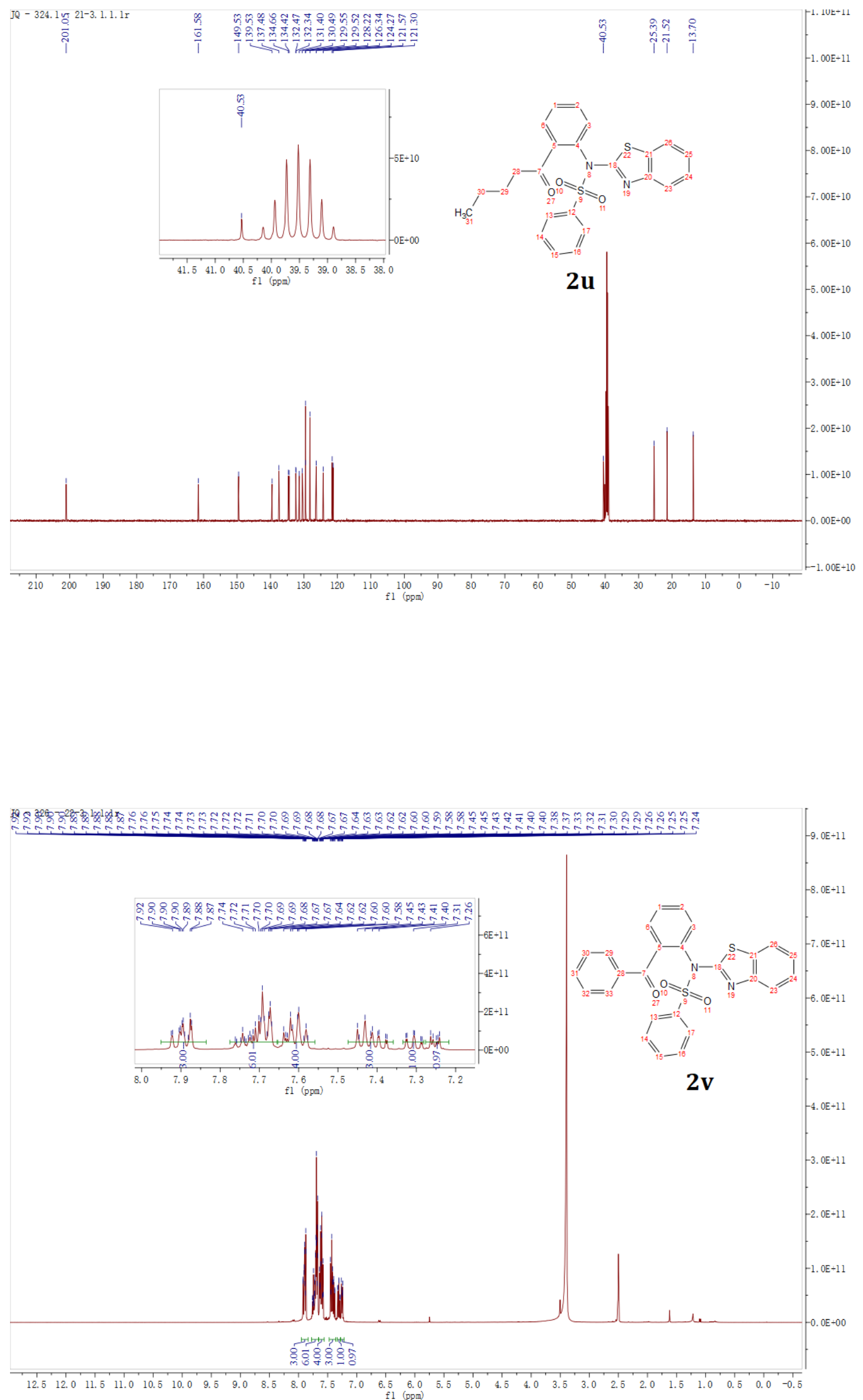

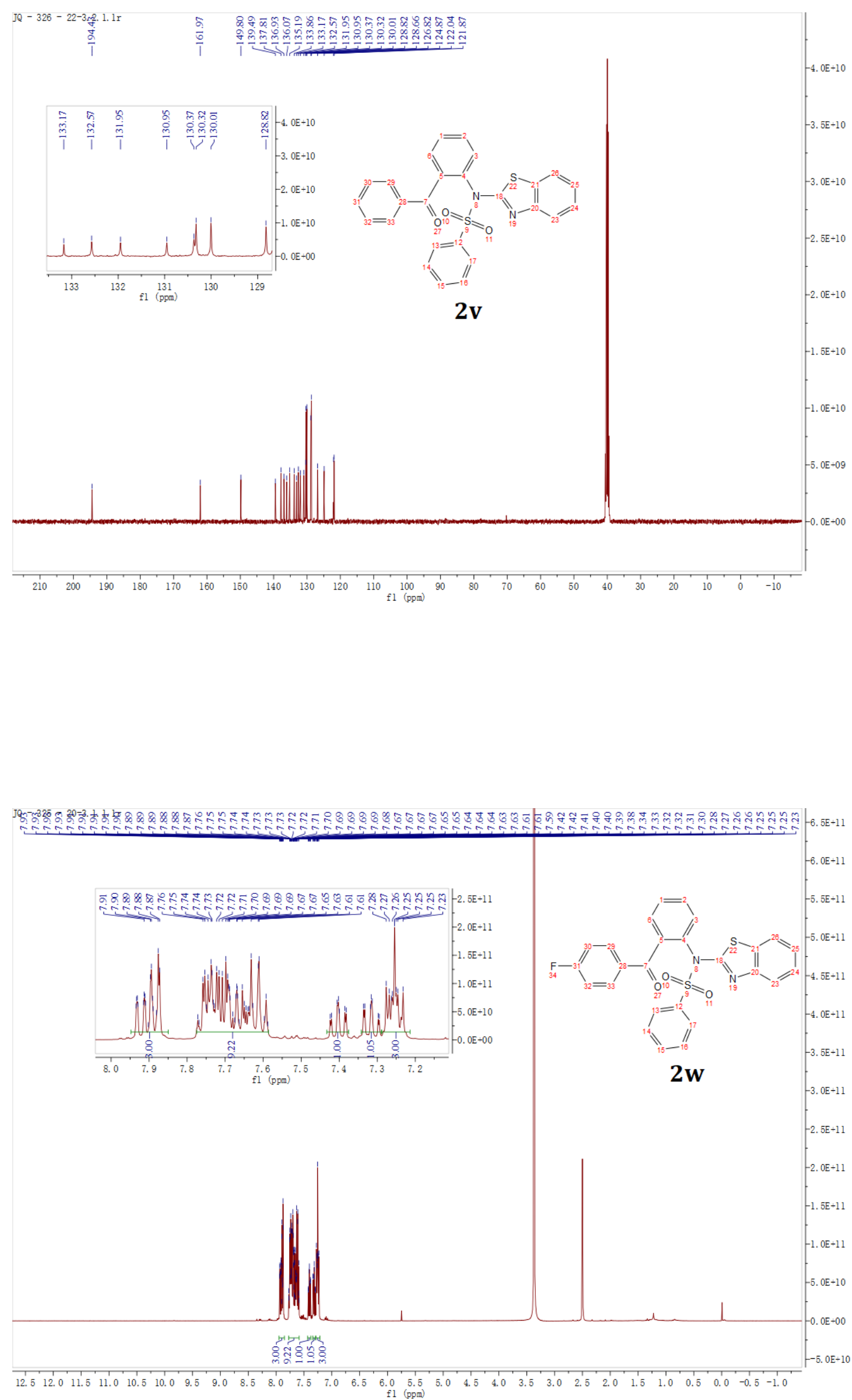

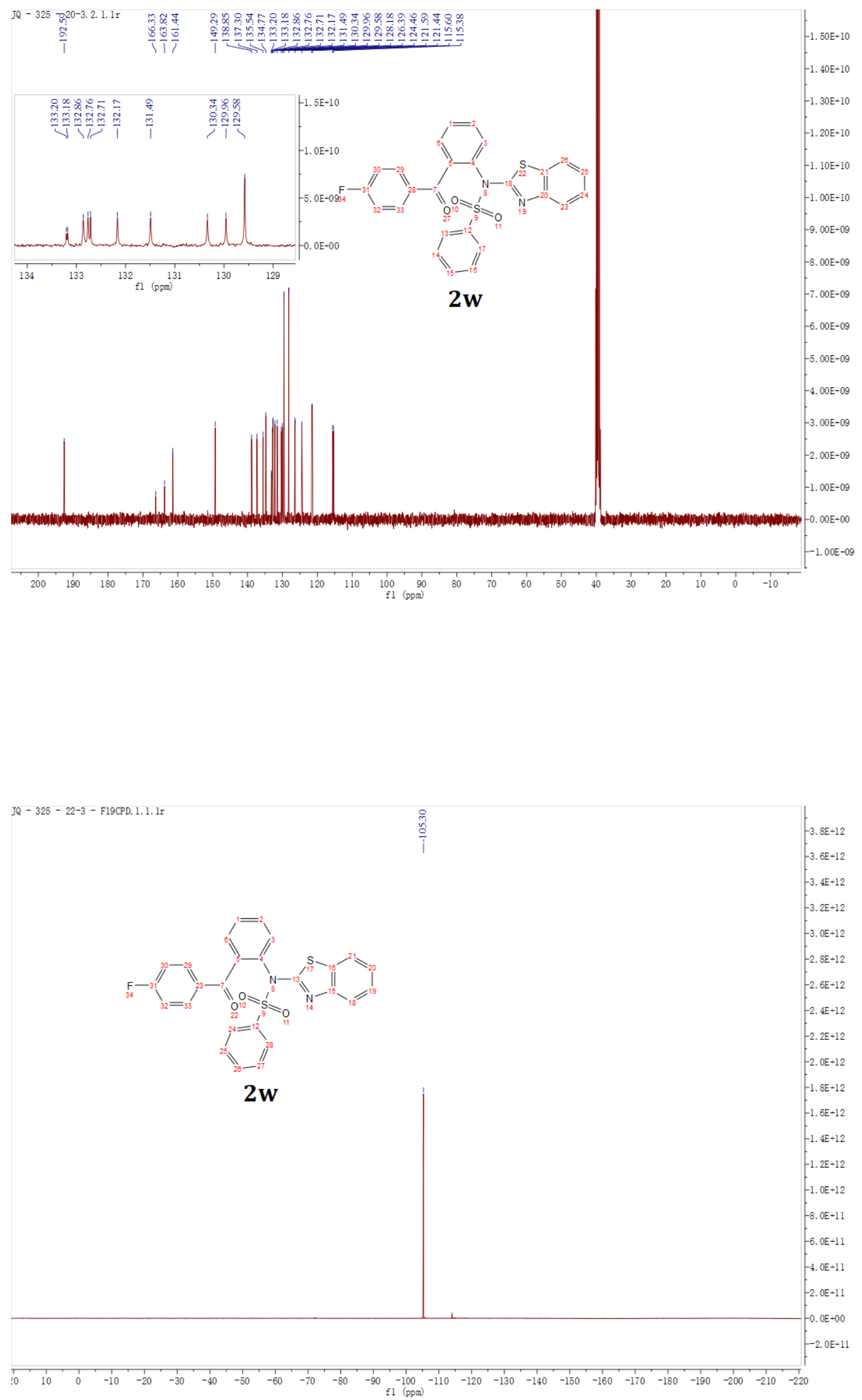